LU TP 13-11

October 2013

\title{
Chiral-Symmetric Technicolor with Standard Model Higgs boson
}

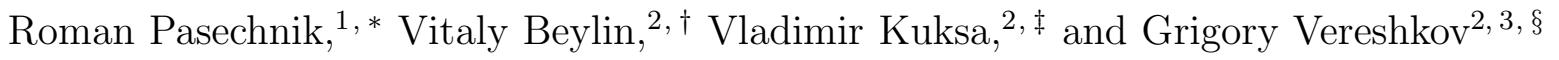 \\ ${ }^{1}$ Department of Astronomy and Theoretical Physics, \\ Lund University, SE-223 62 Lund, Sweden \\ ${ }^{2}$ Research Institute of Physics, Southern Federal University, \\ 344090 Rostov-on-Don, Russian Federation \\ ${ }^{3}$ Institute for Nuclear Research of Russian Academy of Sciences, \\ 117312 Moscow, Russian Federation
}

\begin{abstract}
Most of the traditional Technicolor-based models are known to be in a strong tension with the electroweak precision tests. We show that this serious issue is naturally cured in strongly coupled sectors with chiral-symmetric vector-like gauge interactions in the framework of gauged linear $\sigma$-model. We discuss possible phenomenological implications of such non-standard chiralsymmetric Technicolor scenario in its simplest formulation preserving the Standard Model (SM) Higgs mechanism. For this purpose, we assume the existence of an extra technifermion sector confined under extra $S U(3)_{\mathrm{TC}}$ at the energy scales reachable at the $\mathrm{LHC}, \Lambda_{\mathrm{TC}} \sim 0.1-1 \mathrm{TeV}$, and interacting with the SM gauge bosons in a chiral-symmetric (vector-like) way. In the framework of this scenario, the SM Higgs vev acquires natural interpretation in terms of the condensate of technifermions in confinement in the nearly conformal limit. We study the influence of the lowest lying composite physical states, namely, technipions, technisigma and constituent technifermions, on the Higgs sector properties in the SM and other observables at the LHC. We found out that the predicted Higgs boson signal strengths in $\gamma \gamma$, vector-boson $V V^{*}$ and fermion $f \bar{f}$ decay channels can be sensitive to the new strongly-coupled dynamics and are consistent with the current SM-like Higgs boson observations in the limit of relatively small Higgs-technisigma mixing. At the same time, the chiral-symmetric Technicolor provides us with rich technipion phenomenology at the LHC, and its major implications are discussed in detail.
\end{abstract}

PACS numbers: 14.80.Ec, 14.80.Bn, 12.60.Nz, 14.80.Tt, 12.60.Fr

*Electronic address: Roman.Pasechnik@thep.lu.se

${ }^{\dagger}$ Electronic address: vbey@rambler.ru

${ }_{\ddagger}^{\ddagger}$ Electronic address: vkuksa47@mail.ru

${ }^{\S}$ Electronic address: gveresh@gmail.com 


\section{INTRODUCTION}

A complete experimental verification of the Standard Model (SM), including the discovery of the Higgs boson and precision tests of its properties, is the most intriguing and challenging task of high energy particle physics at the moment. Last year, the major LHC collaborations, ATLAS and CMS 1, 2], have announced the discovery of a new "Higgs-like" particle with the mass of $125.3 \pm 0.6 \mathrm{GeV}$, which may become the last yet missing piece predicted within the SM framework - the Higgs boson. Some evidence for the Higgs boson has been also seen by CDF and D0 collaborations at the Tevatron [3].

An ultimate proof of the Higgs boson's existence and understanding of its nature would only be possible after high precision measurements of its decay parameters which can be sensitive to details of a particular New Physics scenario. The current situation with the Higgs boson properties suggests that there are no significant deviations from the SM (within rather large statistical and systematical uncertainties) as revealed by the full data set collected so far at the LHC [4] and Tevatron [5] (for the most recent comprehensive studies of the Higgs boson properties, see e.g. Refs. [6 9] ). Even though the room for New Physics contributions has been greatly reduced [7, 11], it is too early to draw final conclusions about the properties and nature of the newly discovered particle not only due to large experimental error bars, but also due to theoretical uncertainties in the SM Higgs production which are rather high and become dominant [8, 12]. If the branching ratios deviate from predictions of the simplest one-doublet SM, even slightly, this would require a proper extension of the SM and pose a serious question about theoretical principles such an extension should be based upon.

Traditionally, ideas of additional to SM strongly-coupled sectors in confinement were realized in the Technicolor (TC) model which was one of the strongest alternatives to the Higgs mechanism of the spontaneous Electroweak Symmetry Breaking (EWSB) [13]. The existing Higgs-less TC models with dynamical EWSB (DEWSB) are based upon the idea that the Goldstone degrees of freedom (technipions) appearing after the global chiral symmetry breaking $S U(2)_{L} \otimes S U(2)_{R} \rightarrow S U(2)_{W}$ are absorbed by the SM weak gauge bosons which thereby gain masses. The DEWSB mechanism is then triggered by the condensate of technifermions in confinement, $\langle\tilde{Q} \overline{\tilde{Q}}\rangle \neq 0$. Traditional TC models with DEWSB are faced with the problem of the mass generation of standard fermions, which was consistently resolved in Extended TC scenarios [14]. However, many of the existing TC models have got severely constrained or even ruled out by the EW precision data [15] (for a detailed review on the existing TC models, see e.g. Refs. [16, 17]). Generally, in these schemes noticeable contributions to strongly constrained Flavor Changing Neutral Current (FCNC) processes appear together with too large contributions to Peskin-Takeuchi (especially, to $S$ ) parameters. Further developments of the TC ideas have resulted in the Walking TC model which succeeded in resolving the above-mentioned problems and remains a viable model of the DEWSB [18 20].

Very recently, as was shown in Ref. [1] based on the latest LHC data, the $1 \sigma$ allowed region of the relative to SM-predicted Higgs-vector-vector fusion $H V V$ coupling is $0.96_{-0.15}^{+0.13}$, which sets further constraints on the EWSB models alternative to the SM Higgs mechanism, as well as to composite Higgs models (see also current bounds on the rescaling of the SM couplings in Ref. [9, 10]). However, even if the newly-discovered particle is indeed the SM Higgs boson and the Higgs mechanism is experimentally confirmed, all available LHC and high precision EW data do not completely exclude the existence of a strongly-coupled fermion sector in confinement, additional to the SM fermion sector, with a confinement 
scale, $\sim 0.1-1 \mathrm{TeV}$, being not very far from the EW scale $M_{\mathrm{EW}} \sim 100 \mathrm{GeV}$. The main goal of this paper is to prove this statement and to study a new class of viable realistic models for an extra strongly-coupled sector assisting the conventional SM Higgs mechanism at accessible energy scales, along with the study of their implications to the ongoing New Physics searches at the LHC.

An alternative class of TC models usually referred to as bosonic TC scenarios include both a Higgs doublet $H$ and a new TC sector [21 23], without referring to an origin of the Higgs doublet. Most recent realization of the bosonic TC is based upon holographic ideas [24], and allows to explain the existence of recently discovered Higgs-like $125 \mathrm{GeV}$ particle and its possible non-standard features [25]. In this approach, strongly coupled dynamics is defined using the AdS/CFT correspondence within the holographic approach allowing to avoid the EW precision constraints [26 28]. In contrast to conventional (Extended and Walking) TC models, in bosonic TC models the mechanism of the EWSB and generation of SM fermions masses is driven by the Higgs vacuum expectation value (vev) in the standard way, irrespectively of (elementary or composite) nature of the Higgs field itself. Due to linear source term in the Higgs potential the Higgs field $H$ develops vev which in turn is induced by the technifermion condensate. This means the Higgs mechanism is not the primary source of the EWSB, but effectively induced by an unknown TC dynamics at high scales. For more alternatives on TC and compositeness models, see e.g. Ref. [29].

In this work, we start off with the similar ideas about the existence of an extra Higgs-like scalar field and TC nature of the SM Higgs vev implemented in the bosonic TC models and study theoretical and phenomenological opportunities of new possible strongly coupled sectors with chiral-symmetric (vector-like) gauge interactions. We further develop these ideas based on the gauged linear $\sigma$-model [36 38] and applied it to new TC-induced degrees of freedom, in a complete analogy with low-energy hadron physics applications. In this model, which will further be referred to as the Chiral-Symmetric (or Vector-Like) Technicolor (in short, CSTC) scenario, the oblique (Peskin-Takeuchi) parameters and FCNC corrections turn out to be naturally very small and fully consistent with the current EW constraints as well as with the most recent Higgs couplings measurements at the LHC in the limit of small Higgs-technisigma mixing. Most importantly, this happens naturally in the standard quantum-field theory framework implemented in rigorous quark-meson approaches of hadron physics without attracting any extra holographic or other special arguments from unknown high-scale physics. For simplicity, we adopt the simplest version of the Standard Model with one Higgs doublet, and the question whether it is elementary or composite is not critical for further considerations. The new heavy physical states of the model (additional to those in the SM) are the singlet technisigma $\tilde{\sigma}$, triplet of technipions $\tilde{\pi}_{a}, a=1,2,3$, and constituent technifermions $\tilde{Q}$ which acquire masses via the technifermion condensate as an external source and the technisigma vev (other composite degrees of freedom are usually much heavier and decoupled from the considering low-energy limit of the theory). Their possible phenomenological implications and signatures at the LHC is the subject of our analysis.

Despite the phenomenological advantages mentioned above, the proposed CSTC scenario, at least, in its simplest form considered here, does not attempt to resolve the naturalness problem of the SM, i.e. does not provide a mechanism protecting the Higgs boson mass itself from becoming arbitrary large. Nevertheless, it points out a promising path towards a consistent formulation of composite Higgs models in extended chiral-gauge theories with vector- 
like UV completion ${ }^{1}$. Indeed, an existence of composite Higgs-like bosons is often considered as a primary guideline for Technicolor models. In analogy with hadron physics, composite bosons can be of two different types: pseudo-Goldstone collective excitations (quantum wave of correlations between non-perturbative technifermion fluctuations in technivacuum) and techniquarkonia (a "bubble" of technivacuum stabilized by valence technifermions). After LHC experiments, the Technicolor models with composite SM-like Higgs bosons have become favorable. The latter means that the SM-like Higgs mechanism is indeed realized in Nature even though it can be treated as an effective one, i.e. the initial fields of collective excitations or techniquarkonia should be in the fundamental representation of the EW gauge group with hypercharge $Y=1 / 2$. In the CSTC model such objects naturally appear if one extends the technifermion sector. The simplest extension is such that in addition to the EW doublet of technifermions $\tilde{Q}=(U, D)$ one introduces an extra weak-singlet technifermion $S$. Therefore, a new composite scalar field appears $\mathcal{H}=\bar{S} \tilde{Q}$ having transformation properties of the Higgs boson $\left(S U(2)_{\mathrm{W}}\right.$ doublet with $\left.Y=1 / 2\right)$. In this model, the initial classification (techniflavor) group is the global chiral group $S U_{\mathrm{L}}(3) \otimes S U_{\mathrm{R}}(3)$. A further generalization would be to consider $S U_{\mathrm{L}}(4) \otimes S U_{\mathrm{R}}(4)$ giving rise to effective two Higgs-doublet model. Of course, in such extended techniflavor models there appears a plenty of new technihadron states which require a separate lengthy analysis. In analogy to hadron physics one may expect, however, that the lightest physical technihadron states which are the most interesting for the LHC phenomenology in the first place are technipions, technisigma and, in principle, lightest technibaryons. Therefore, in this paper we limit ourselves to considering initial (presumably, the minimal) techniflavor group $S U_{\mathrm{L}}(2) \otimes S U_{\mathrm{R}}(2)$ and discuss a simplified model with gauged vector-like subgroup $S U(2)_{\mathrm{L}+\mathrm{R}}$ only where the Higgs boson formally (at the low-energy part of the spectrum of technihadrons) has a status of the fundamental field, which does not satisfy the naturalness criterium. An extended techniflavor model $S U_{\mathrm{L}}\left(N_{f}\right) \otimes S U_{\mathrm{R}}\left(N_{f}\right)$ with $N_{f}>2$ will be studied elsewhere.

The paper is organized as follows. The Section II is devoted to description of theoretical foundations of the CSTC scenario along with the physical Lagrangian derivation and analysis of the parameter space. The study of EW constraints (oblique corrections and FCNC) is performed in Section III. Some basic opportunities for LHC phenomenology, in particular, in studies of the Higgs sector properties, as well as in searches for new lightest composites, are discussed in Section IV. Finally, Section V summarizes the basic results of the paper.

\section{CHIRAL-SYMMETRIC TECHNICOLOR MODEL}

\section{A. Vector-like technifermions vs chiral SM fermions}

Historically, the Nambu-Jona-Lasinio (NJL) model [32] based on the global chiral group $S U\left(N_{f}\right)_{\mathrm{L}} \otimes S U\left(N_{f}\right)_{\mathrm{R}}$ is the first model describing dynamical breaking of chiral symmetry in

\footnotetext{
${ }^{1}$ Also, the model does not provide a mechanism for generation of current (Dirac) technifermion masses which á priori are arbitrary. In analogy to ordinary QCD, however, we consider the physically interesting conformal limit of the new strongly coupled dynamics realized in the chiral limit of the theory $m_{U, D} \ll \Lambda_{\mathrm{TC}}$ which leads to an unambiguous determination of the Higgs vev in terms of the technifermion condensate. The latter means that the EW symmetry is broken dynamically via the effective Higgs mechanism in this limit, which makes it particularly interesting. This statement is stable w.r.t. radiative corrections.
} 
particles physics (for review on the topic, see e.g. Ref. [33]). A large interest in the gauged version of the NJL model (or GNJL) initially proposed in Ref. [34] has been stimulated by its importance for constructing extended TC models and top-quark condensate models (for an extensive review of the GNJL models and their applications, see Ref. [35]). The GNJL approach has fewer parameters and significantly reduces ambiguities of corresponding predictions.

As one of the most successful implementation of the GNJL ideas in hadron physics, the socalled gauged linear $\sigma$-model (GL $\sigma \mathrm{M})$ initially proposed in Ref. [36] and further elaborated in Refs. [37, 38] was one of the first models with local chiral $S U(2)_{\mathrm{R}} \otimes S U(2)_{\mathrm{L}}$ symmetry, which incorporates the vector $\rho$ and pseudovector $a_{1}$ mesons as corresponding gauge bosons, besides lightest pseudoscalar pion $\pi$ and scalar $\sigma$ fields. Typically, the local chiral symmetry is spontaneously broken by the scalar $\sigma$ vev giving rise to the vector-meson mass terms, constituent light quark masses [39] and the mass splitting between $\rho$ and $a_{1}$ mesons.

In what follows, we employ the ideas of the GL $\sigma \mathrm{M}$ and consider the global chiral $S U\left(N_{f}\right)_{\mathrm{L}} \otimes S U\left(N_{f}\right)_{\mathrm{R}}$ group in the technifermion sector $\tilde{Q}$ in the simplest case with $N_{f}=2$, with its subsequent breaking (by the technisigma vev) down to the vector subgroup $S U(2)_{\mathrm{V} \equiv \mathrm{L}+\mathrm{R}}$ which is then gauged at energy scales close to the EWSB scale. Such a "gauging", however, does not necessarily mean that one should introduce extra gauge bosons to the existing theory. The "gauging" procedure may also mean that corresponding fundamental technifermions interact with already existing gauge bosons in the SM in the low-energy effective field theory limit, which is a rather plausible opportunity we wish to explore here. In analogy with standard QCD and hadron physics, at the scale of the order of the techniconfinement scale $\Lambda_{\mathrm{TC}}$ technifermions acquire effective non-perturbative constituent masses due to the chiral symmetry breaking [39]. At lower energies the initial technifermions condense into technihadron states due to confinement. This scheme is an analogy of the chiral-invariant QHD-III model [38] where the pseudo-Goldstone technipion fields $\tilde{\pi}_{a}$ get the same masses (via an external source term linear in $\tilde{\sigma}$ field) and remain the physical degrees of freedom, in distinction from many other traditional TC and compositeness scenarios.

For the sake of simplicity, we consider a possible scenario of the SM extension by means of an additional chiral-symmetric (vector-like) technifermion sector confined under $S U(3)_{\mathrm{TC}}$ group, which is analogical to the $S U(3)_{c}$ color group of QCD. Such an assignment is not unique, of course, but would allow us to use direct analogies with hadron physics ${ }^{2}$. The GL $\sigma \mathrm{M}$ can therefore be efficiently extended to incorporate constituent technifermiontechnimeson interactions as the simplest way of phenomenological description of the non-

${ }^{2}$ For this purpose, one could choose an extension of the gauge and fermion SM sectors motivated by a reduction from the grand-unified theories (GUT) originating from e.g. superstring-inspired $E_{8} \otimes E_{8}^{\prime}$ group with many appealing features [30]. In the latter case, one of the exceptional groups, say, $E_{8}^{\prime}$ can exist in confinement and, possibly, consists of a few unbroken subgroups confined at different scales, whereas the second $E_{8}$ gets broken down to the SM gauge group $G_{\mathrm{SM}} \equiv S U(3)_{c} \otimes S U(2)_{\mathrm{W}} \otimes U_{\mathrm{Y}}(1)$ in a straightforward way. As a realistic possibility, one of the $S U(3)$ subgroups of the original $E_{8}^{\prime}$ can be, in principle, identified with the TC gauge group $S U(3)_{\mathrm{TC}}$, which acts only on new additional technifermion sector, and there are no any obstacles for it to be confined at relatively low scales being not very far from the EWSB scale (later it will be shown that the latter condition is not critical for the TC-induced EWSB). 
perturbative effects in technihadron dynamics at low energies. We will further refer to it below as the gauged linear technisigma model, or GLT $\sigma \mathrm{M}$. In the simplest version of this model, the non-perturbative effects are accounted for by an effective NJL-type theory of constituent technifermion interactions with the lightest technihadron states only [39] - technipions and technisigma. In the context of GLT $\sigma \mathrm{M}$ we suggest the following hypothesis, which will be studied below: the energy scales of the EWSB and techni-confinement have a common quantum-topological nature and are determined by a non-perturbative dynamics of the technifermion-technigluon condensate. In particular, we would like to find specific conditions on the model parameters under which the latter hypothesis is validated. As was noted above, the technipion d.o.f. $\tilde{\pi}_{a}$ are the pseudo-Goldstone fields which are usually considered as collective fluctuations of the technifermion-technigluon vacuum, while technisigma $\tilde{\sigma}$ is the lightest techniglueball state - these states are not usual bound $\tilde{Q} \tilde{\tilde{Q}}$ states and thus play a special role in the GLT $\sigma \mathrm{M}$ [36 38].

From the point of view of the GLT $\sigma \mathrm{M}$, the spontaneous breaking of the global chiral symmetry group in the technifermion sector happens in the chiral-symmetric (vector-like) way in a complete analogy with the chiral symmetry breaking in GNJL models [35, 38] as follows

$$
S U(2)_{\mathrm{L}} \otimes S U(2)_{\mathrm{R}} \rightarrow S U(2)_{\mathrm{V} \equiv \mathrm{L}+\mathrm{R}} \equiv S U(2)_{\mathrm{W}},
$$

where the subsequent gauging of the resulting unbroken vector subgroup $S U(2)_{\mathrm{V}}$ and its identification with the weak gauge group of the SM are performed. Such gauging and identification procedures are not forbidden theoretically and lead to specific properties of the technifermion sector, which thereby make it to be very different from the chiral-nonsymmetric SM fermion sectors. It therefore means that after the chiral symmetry breaking in the technifermion sector the left and right components of the original Dirac technifermion fields can interact with the $\mathrm{SM}$ weak $S U(2)_{\mathrm{W}}$ gauge bosons with vector-like couplings, in opposition to ordinary SM fermions, which interact under $S U(2)_{\mathrm{W}}$ by means of their left-handed components only. Note, analogous vector-like gauge interactions are rather common and appear e.g. in the chargino sector of the MSSM.

Note, the above procedure (2.1) should be understood in exactly the same way as is done in the QCD hadron physics at low energies. There, the fundamental gauge group of color $S U(3)_{c}$ is vector-like i.e. acts on left-handed $q_{L}$ and right-handed $q_{R}$ quarks in exactly the same way, which makes it possible to introduce the global chiral group $S U(3)_{\mathrm{L}} \otimes S U(3)_{\mathrm{R}}$. The latter is typically broken down to the vector-like subgroup $S U(3)_{\mathrm{V} \equiv \mathrm{L}+\mathrm{R}}$ by the $\sigma$-vev. If one gauges it, one recovers that its properties are identical to the color group $S U(3)_{c}$ in the low energy limit. This leads to a low-energy effective field theory where interaction properties of elementary and composite fields are effectively described by the same gauge group with renormalized local gauge couplings (as limiting values of corresponding form factors valid at small momentum transfers). Similarly, vector-like weak interactions of technifermions make it possible to introduce the chiral group whose gauged subgroup has properties identical to the weak isospin group (2.1). Most importantly, the latter procedure is valid only in the phenomenologically interesting low energy limit of the theory. When typical momentum transfers become comparable to the techniconfinement scale or larger $Q^{2} \gtrsim \Lambda_{\text {TC }}^{2}$ the global chiral symmetry is fully restored, while fundamental EW gauge interactions of technifermions remain vector-like (similarly to QCD interactions of quarks in perturbative limit).

So, in this scenario the sector of initial (current) technifermions transforms according to the local gauge $S U(2)_{\mathrm{W}} \otimes U_{\mathrm{Y}}(1)$ symmetry group, and, therefore, interacts only with SM 
gauge bosons $B, W^{a}, a=1,2,3$, or with $W^{ \pm}, Z^{0}$ and $\gamma$ after the SM symmetry breaking. Of course, in a complete local chiral $S U(2)_{\mathrm{L}} \otimes S U(2)_{\mathrm{R}}$ theory of technifermion-technimesongauge interactions one would need to include e.g. a mixing of vector technirho $\tilde{\rho}$ with the elementary SM gauge bosons as is done in local quark-meson interaction theories [38, 40] (for review on the subject, see also Ref. [41] and references therein). However, in this work in what follows we neglect the heavier vector and pseudovector technimesons, such that only elementary gauge $B, W^{a}, a=1,2,3$ fields remain, and consider only the spectrum of lightest composite scalar (technisigma $\tilde{\sigma}$ ) and pseudoscalar (technipion $\tilde{\pi}_{a}$ ) states, relevant for the LHC measurements. Note, that a reduction scheme to the left-right (LR) symmetric subgroup $S U(2)_{\mathrm{L}+\mathrm{R}}$ enables one to introduce current masses of technifermions directly into the initial Lagrangian without a need in extra fields which is considered to be advantageous. While the latter freedom may be regarded as a new form of the hierarchy problem as there must be a symmetry which protects the current up $(U)$ and down $(D)$ technifermion masses $m_{U, D}$ from becoming very large, we take on the phenomenological approach and consider the chiral limit of the theory with the current masses being small compared to the techniconfinement energy scale, i.e. $m_{U, D} \ll \Lambda_{\mathrm{TC}}$, in a complete analogy with the chiral QCD framework. Surely, the latter issue should be addressed in a high-scale GUT-like theory which incorporates new strongly-coupled fermion sectors, and this certainly goes beyond the scope of the present analysis. Additionally, chiral (axial) anomalies do not appear in this framework; it is anomaly-safe automatically. We will further discuss specific consequences of such new vector-like weak interactions of the additional technifermion sector in confinement.

One should remember that identification of the local vector subgroup of the chiral group with the SM weak isospin group (2.1) is a purely phenomenological procedure which leads to correct results in the low energy limit of the theory. In reality, of course, the global classification techniflavor group $S U_{\mathrm{L}}(2) \otimes S U_{\mathrm{R}}(2)$ has nothing to do with the EW gauge group of the SM. At the first stage, the techniflavor group is used for classification of composite technihadrons and, in particular, predicts the existence of technipions, technisigma and technibaryons states. At the second stage, one notices that technifermions entering the composite technihadrons besides technistrong interactions participate also in the fundamental EW interactions. One should therefore calculate the $E W$ form factors of composite technihadrons. The corresponding EW interactions must then be also introduced at the fundamental technifermion level consistently with those at the composite level technihadron level. At the third stage, in the phenomenologically interesting low-energy limit of the theory the EW form factors approach the renormalized EW constants (since the technihadron substructure does not emerge at relatively small momentum transfers). The latter should be calculated after reclassification of technihadrons under the EW group representations. This three-fold generic scheme will be used below for description of EW interactions of technihadrons.

According to the standard quark-meson approaches [39, 41], constituent quark loops describe non-perturbative effects at relatively small distances, whereas meson loops work at larger distances. This scheme should be realized in the CSTC model under discussion, in a complete analogy with the standard quark-meson theories, and is valid up to an energy scale of typical technihadron states. Following to this analogy, we consider meson (technipions $\tilde{\pi}_{a}$ and technisigma $\tilde{\sigma}$ ) interactions at tree level, and technifermion interactions (with effective constituent masses) at one-loop level [39]. At much larger energies, one should turn into the perturbative techni-QCD framework describing technigluon and technifermion (with current masses) interactions, in analogy with the standard QCD approach. 
This scenario becomes especially interesting from both theoretical and phenomenological points of view since it predicts the existence of the physical technimeson spectrum with relatively light pseudoscalar $\tilde{\pi}_{a}$ and scalar $\tilde{\sigma}$ fields ${ }^{3}$. Latter has quantum numbers identical to the SM Higgs boson ones. This leads to a mixing of initial $\tilde{\sigma}$ and $H$ fields causing a possible modification of the physical Higgs boson couplings. Additionally, lightest physical technipion states $\tilde{\pi}_{a}$ enrich LHC phenomenology with possible new observable signatures, to be studied in detail.

\section{B. Gauged linear technisigma model: initial CSTC Lagrangian}

As was shortly discussed in the previous Section, we use the standard structure of the gauged linear $\sigma$-model for low-energy TC phenomenology. Let us formulate the CSTC model in terms of the lightest composite states based on the local weak isospin symmetry group $S U(2)_{\mathrm{L}+\mathrm{R}}=S U(2)_{\mathrm{W}}$ acting on the confined technifermion sector. The initial field content of the CSTC model in its simplest formulation is given by one $L R$-symmetric doublet of technifermions

$$
\tilde{Q}=\left(\begin{array}{c}
U \\
D
\end{array}\right)
$$

which forms the fundamental representation of the $S U(2)_{\mathrm{W}} \otimes U(1)_{\mathrm{Y}}$ group, the initial scalar technisigma $S$ field which is the singlet representation, and the triplet of initial technipion fields $P_{a}, a=1,2,3$ which is the adjoint (vector) representation of $S U(2)_{\mathrm{W}}$ (with zeroth $U(1)_{\mathrm{Y}}$ hypercharge). Thus, in terms of the fields introduced above the GLT $\sigma \mathrm{M}$ part of the Lagrangian responsible for Yukawa-type interactions of the technifermions reads

$$
\mathcal{L}_{Y}^{\mathrm{CSTC}}=-g_{\mathrm{TC}} \overline{\tilde{Q}}\left(S+i \gamma_{5} \tau_{a} P_{a}\right) \tilde{Q}
$$

where $\tau_{a}, a=1,2,3$ are the Pauli matrices. By restricting ourselves to considering only one technifermion doublet (2.2) (the first generation), we imply that other generations, if exist, are much heavier and split off in the mass spectrum, based on analogy with the SM, even though such an analogy is not mandatory.

In the SM, the gauge boson interactions with usual hadrons are typically introduced by means of hadronisation effects (see Fig. 1 (left)). In our case, such an effect is strongly
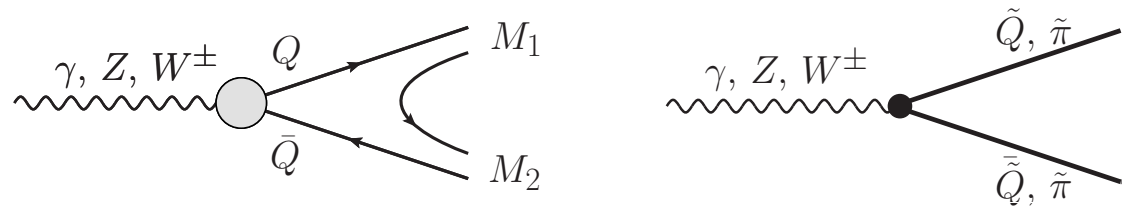

FIG. 1: An illustration of the interactions of (techni)fermion and (techni)meson fields with the SM gauge bosons via (techni)hadronisation in hadron physics (left panel) and in the point-like approximation adopted in the considered CSTC scenario (right panel).

\footnotetext{
${ }^{3}$ It is typically assumed that technibaryons, along with the vector and pseudovector states, are much heavier and thus likely to be irrelevant for the LHC phenomenology, at least, at the moment. Although if the techni-confinement scale $\Lambda_{\mathrm{TC}}$ is not very far above the EW scale, technibaryon states might emerge in LHC data as large missing $E_{T}$ signatures which is a subject for further studies.
} 
suppressed by large constituent masses of technifermions $\sim \Lambda_{\mathrm{TC}}$. Instead, the interactions of $\tilde{Q}$ and $P_{a}$ fields with initial SM gauge fields $B_{\mu}, V_{\mu}^{a}$ can be introduced via the local approximation which is illustrated in Fig. 1 (right). Generally speaking, these interactions should be written in terms of nonlocal form factors since both technimesons and dressed (constituent) technifermions are the objects delocalized at energy scales exceeding the scale of non-perturbative technigluon fluctuations. We assume, however, that the latter scale is large compared to the EWSB scale and corresponding effects can be neglected at experimentally accessible energy scales. Thus, in the first approximation one can replace the form factors by point-like couplings as is usually done in the local quantum-field theory approach ${ }^{4}$. The coupling constants of $\widetilde{Q}$ and $P_{a}$ with gauge fields can be taken the same as in the SM, but calculated via the Renormalisation Group evolution at corresponding scales. Since this evolution is logarithmic and rather weak, whereas $\Lambda_{\mathrm{TC}}$ is assumed to be in a vicinity of the EW scale, in the leading-order numerical analysis below we fix all the relevant couplings at the $M_{Z}$ scale.

The vector-like gauge interactions can be introduced via covariant derivatives over the local $S U(2)_{\mathrm{W}} \otimes U(1)_{\mathrm{Y}}$ group in the same form as the SM gauge interactions, i.e. the additional (to the SM) kinetic terms have the following form

$$
\mathcal{L}_{\text {kin }}^{\mathrm{CSTC}}=\frac{1}{2} \partial_{\mu} S \partial^{\mu} S+\frac{1}{2} D_{\mu} P_{a} D^{\mu} P_{a}+i \overline{\tilde{Q}} \hat{D} \tilde{Q}
$$

where the covariant derivatives of the $\tilde{Q}$ and $P_{a}$ fields reads

$$
\hat{D} \tilde{Q}=\gamma^{\mu}\left(\partial_{\mu}-\frac{i Y_{\tilde{Q}}}{2} g^{\prime} B_{\mu}-\frac{i}{2} g W_{\mu}^{a} \tau_{a}\right) \tilde{Q}, \quad D_{\mu} P_{a}=\partial_{\mu} P_{a}+g \epsilon_{a b c} W_{\mu}^{b} P_{c}
$$

respectively. Further, we wish to employ analogies with the SM and, in particular, with QCD as much as possible, so for the sake of convenience and simplicity in actual calculations we fix the hypercharge of the technifermion doublet (2.2) to be the same as that of the quark doublet in the SM, i.e. $Y_{\tilde{Q}}=1 / 3$, unless noted otherwise. Certainly, the hypercharge $Y_{\tilde{Q}}$, the number of technifermion generations, the respective properties of interactions, etc. should be ultimately constrained in extended chiral-gauge or grand-unified theories incorporating extra technifermion sectors, which is a subject of further studies.

In Eqs. (2.4) and (2.5) we notice two key differences of the CSTC scenario from traditional TC-based models (cf. Ref. [15, 29]) - the existence of physical technisigma and technipion states, introduced via the GLT $\sigma \mathrm{M}$ approach, and the equivalence of left and right technifermion chiralities in their interactions with weak gauge bosons, following from the gauging of the initial chiral group of the linear $\sigma$ model. Along with the absence of chiral anomalies, the CSTC scenario under discussion can be considered as a solid theoretically motivated basis for the whole new class of more elaborated TC-based extensions of the SM and their phenomenological tests.

Next, let us consider the potential part of the CSTC model Lagrangian giving rise to (pseudo)scalar self-interactions and $\tilde{\pi}, \tilde{\sigma}$ masses after the chiral symmetry breaking and the EWSB. As was mentioned in the Introduction, in the simplest formulation of the CSTC

\footnotetext{
${ }^{4}$ In a more rigorous analysis this approximation can be easily lifted by introducing the Pauli form factors, although in this very first analysis of the CSTC we work in the point-like approximation for the sake of simplicity.
} 
model developed in this work we keep the SM Higgs mechanism of the EWSB and the one-Higgs-doublet SM untouched, and simply add extra technifermion sector (2.2) in confinement. As an essential part of the CSTC model, we introduce the interaction terms between the standard Higgs doublet $\mathcal{H}$, and the new $P_{a}$ and $S$ states which are allowed by the local $S U(2)_{W}$ symmetry. As will be demonstrated below, such extra terms lead to a mixing between the scalar Higgs and technisigma fields. The most general form of the Lagrangian corresponding to the scalar self-interactions including $\mu$-terms as follows [41]

$$
\mathcal{L}_{U, \text { self }}^{\mathrm{CSTC}}=\frac{1}{2} \mu_{\mathrm{S}}^{2}\left(S^{2}+P^{2}\right)+\mu_{\mathrm{H}}^{2} \mathcal{H}^{2}-\frac{1}{4} \lambda_{\mathrm{TC}}\left(S^{2}+P^{2}\right)^{2}-\lambda_{\mathrm{H}} \mathcal{H}^{4}+\lambda \mathcal{H}^{2}\left(S^{2}+P^{2}\right)
$$

and the extra linear "source" term which appears after averaging over the technifermion vacuum fluctuations and describes interactions of the scalar singlet $S$ field with scalar modes of the technifermion condensate, i.e.

$$
\mathcal{L}_{U, \text { source }}^{\mathrm{CSTC}}=-g_{\mathrm{TC}} S\langle\overline{\tilde{Q}} \tilde{Q}\rangle .
$$

The potential part of the GLT $\sigma$ M Lagrangian is then given by

$$
\mathcal{L}_{U}^{\mathrm{CSTC}}=\mathcal{L}_{U, \text { self }}^{\mathrm{CSTC}}+\mathcal{L}_{U, \text { source }}^{\mathrm{CSTC}}
$$

In Eq. (2.6) we defined $P^{2} \equiv \sum_{a} P_{a} P_{a}=\tilde{\pi}^{0} \tilde{\pi}^{0}+2 \tilde{\pi}^{+} \tilde{\pi}^{-}$, whereas gauge-Higgs interaction terms are the same as in the SM.
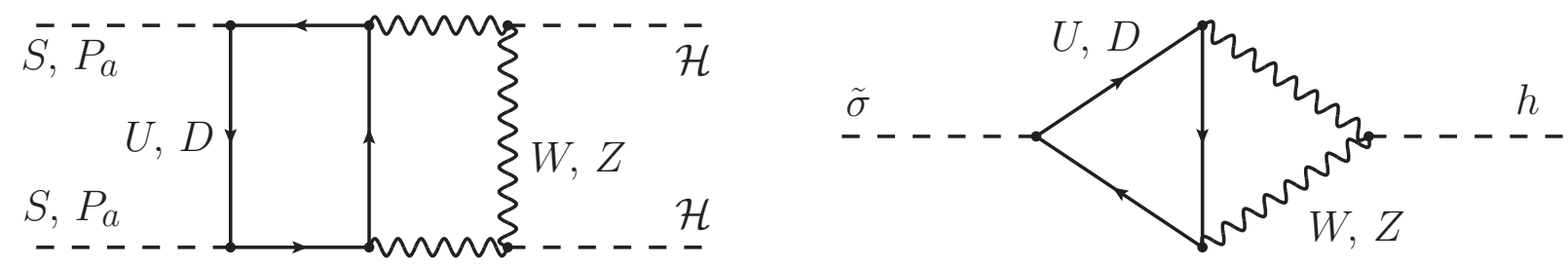

FIG. 2: Typical radiative corrections to the quartic Higgs-TC coupling $\lambda$ (in particular, giving rise to the $h \tilde{\sigma}$-mixing) before the EWSB (left) and after the EWSB (right).

The mixing between the Higgs boson and scalar technisigma fields is governed by the quartic Higgs-TC coupling $\lambda$ in Eq. (2.6). Such a mixing is one of the characteristic effects of the chiral-symmetric Technicolor. In a sense, this effect is indeed one of the motivations of the model under discussion. It has to be taken into consideration if the precision LHC measurements uncover possibly small deviations of the Higgs-like $126 \mathrm{GeV}$ boson (especially, in the $\gamma \gamma$ decay channel) from the standard Higgs boson. The quartic coupling $\lambda$ controls such a mixing and á priori is allowed by the gauge symmetry of the initial Lagrangian, thus, cannot be identically equal to zero. Indeed, any terms which are allowable by the initial symmetry of the model, even being equal to zero at the tree level, necessarily appear in divergent radiative corrections. In order to renormalize such divergencies one has to introduce corresponding counterterms. So if at a given scale $\mu_{0}$ the coupling $\lambda\left(\mu_{0}\right) \rightarrow$ 0 vanishes it will reappear at another scale. In particular, before the spontaneous EW symmetry breaking the operator $\sim \mathcal{H}^{2}\left(S^{2}+P^{2}\right)$ is supported by the two-loop box-box diagram illustrated in Fig. 2 (left) with incoming initial $S$ and $P_{a}$ fields and outgoing initial Higgs field $\mathcal{H}$. This operator thus contributes to remormalization of $\lambda$ coupling. After 
the EWSB, the resulting physical $h \tilde{\sigma}$ mixing is renormalized by two-loop triangle-triangle diagram shown in Fig. 2 (right) ${ }^{5}$. In extended $S U_{\mathrm{L}}\left(N_{f}\right) \otimes S U_{\mathrm{R}}\left(N_{f}\right)$ models mentioned above the corresponding quartic Higgs-TC operator which mixes physical $h$ and $\tilde{\sigma}$ appears automatically from the main invariant of the linear $\sigma$-model and cannot be eliminated.

In order to provide the EWSB and the chiral symmetry breaking in the simplest way, the Higgs $\mathcal{H}$ and technisigma $S$ fields get vevs and corresponding physical scalar degrees of freedom are mixed up, i.e.

$$
\begin{array}{cl}
\mathcal{H} & =\frac{1}{\sqrt{2}}\left(\begin{array}{c}
\sqrt{2} i \phi^{-} \\
H+i \phi^{0}
\end{array}\right), \quad H=v+h c_{\theta}-\tilde{\sigma} s_{\theta}, \quad\langle\mathcal{H}\rangle=\frac{1}{\sqrt{2}}\left(\begin{array}{l}
0 \\
v
\end{array}\right), \\
v & =\frac{2 M_{W}}{g} \simeq 246 \mathrm{GeV}, \quad S=u+h s_{\theta}+\tilde{\sigma} c_{\theta}, \quad\langle S\rangle=u \gtrsim v,
\end{array}
$$

where $M_{W}$ is the $W$ boson mass, $v, u$ are the Higgs boson and technisigma $\tilde{\sigma}$ vevs; $h, \tilde{\sigma}$ are the corresponding physical fields with positively definite masses $M_{h}, M_{\tilde{\sigma}}$, respectively; $c_{\theta} \equiv \cos \theta, s_{\theta} \equiv \sin \theta$, and $\theta$ is the mixing angle, which diagonalizes the respective scalar mass form. We therefore end up with the physical Lagrangian which describes new types of interactions, namely, between Higgs boson, technipions and technisigma, Yukawa technifermion interactions, as well as mixing effects between the Higgs boson and technisigma fields, relevant for the LHC phenomenology.

As it is well-known, in the SM framework we deal with two energy scales of a completely different nature. The first one is the scale of quark-gluon condensate which has a quantumtopological nature. The second one given by the amplitude of the constant Higgs field (vev) has classical (non-quantum) origin. In the framework of the CSTC model we suggest another interpretation of the classical Higgs mechanism in which the nature of all energy scales (including the Higgs vev) is quantum-topological, in the essence of original TC and compositeness models of the DEWSB. The simplest way to realize this idea is to introduce into the scalar potential an "external source" term (the first term in Lagrangian (2.8) linear in $S$ field) which describes interactions between technifermion condensate with the singlet scalar $S$ field [41]. As will be demonstrated below, in the framework of the CSTC model this term leads to a close connection between the Higgs and technifermion condensates. A possible experimental verification of the CSTC model at the LHC relies on our assumption that both EW and TC scales are relatively close to each other, within the LHC energy scales. Indeed, in this case it is natural to assume that the Higgs and technifermion condensates ( $v$ and $u$, respectively) may have the same origin. Our specific goal is to study possible observable effects of such a phenomenon related, in particular, to the Higgs boson properties as well as to lightest technihadron phenomenology at the LHC energy scales.

\section{Parameter space of the CSTC model}

As was mentioned above, in the framework of CSTC scenario it is assumed that the EWSB in the SM sector (via ordinary Higgs mechanism by the Higgs vev, $v$ ) and the chiral symmetry breaking in the TC sector (via the scalar technisigma field vev, $u$ ) may happen

\footnotetext{
${ }^{5}$ In addition, there is an extra one-loop contribution to the $h \tilde{\sigma}$-mixing which is going via a technipion loop. The latter correction exists for non-zeroth tree-level $\lambda_{\text {tree }} \neq 0$ only.
} 
at energy scales relatively close to each other, i.e. $u \sim \Lambda_{\mathrm{TC}} \sim 0.1-1 \mathrm{TeV}$. In what follows, we adopt this limiting case where one may expect possible specific signatures of the chiralsymmetric strongly coupled sectors potentially observable at the LHC.

Minimizing the potential (2.8) using expressions (2.9) one arrives at the set of tadpole equations for the vacuum expectation values

$$
\begin{gathered}
\left\langle\delta \mathcal{L}_{U}^{\mathrm{CSTC}} / \delta \mathcal{H}\right\rangle=v\left(\mu_{\mathrm{H}}^{2}-\lambda_{\mathrm{H}} v^{2}+\lambda u^{2}\right)=0 \\
\left\langle\delta \mathcal{L}_{U}^{\mathrm{CSTC}} / \delta S\right\rangle=u\left(\mu_{\mathrm{S}}^{2}-\frac{g_{\mathrm{TC}}\langle\bar{Q} Q\rangle}{u}-\lambda_{\mathrm{TC}} u^{2}+\lambda v^{2}\right)=0 .
\end{gathered}
$$

The solution of the above equations with respect to scalar fields vevs has the following form

$$
\begin{gathered}
v^{2}=\frac{\lambda_{\mathrm{TC}} \mu_{\mathrm{H}}^{2}+\lambda\left(\mu_{\mathrm{S}}^{2}+m_{\tilde{\pi}}^{2}\right)}{\lambda_{\mathrm{TC}} \lambda_{\mathrm{H}}-\lambda^{2}}, \\
u^{2}=\frac{\lambda_{\mathrm{H}}\left(\mu_{\mathrm{S}}^{2}+m_{\tilde{\pi}}^{2}\right)+\lambda \mu_{\mathrm{H}}^{2}}{\lambda_{\mathrm{TC}} \lambda_{\mathrm{H}}-\lambda^{2}},
\end{gathered}
$$

where

$$
m_{\tilde{\pi}}^{2}=-\frac{g_{\mathrm{TC}}\langle\overline{\tilde{Q}} \tilde{Q}\rangle}{u}, \quad\langle\overline{\tilde{Q}} \tilde{Q}\rangle<0, \quad g_{\mathrm{TC}}>0
$$

is the technipion mass squared proportional to the (negative-valued) technifermion condensate $\langle\bar{Q} \tilde{Q}\rangle$, similarly to that in low-energy hadron physics. The vacuum stability is ensured

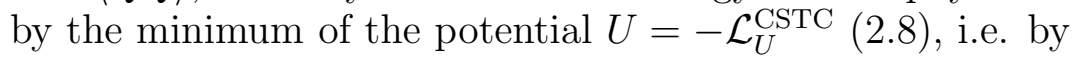

$$
\Delta \equiv\left\langle\frac{\delta^{2} \mathcal{L}_{U}^{\mathrm{CSTC}}}{\delta \mathcal{H} \delta S}\right\rangle^{2}-\left\langle\frac{\delta^{2} \mathcal{L}_{U}^{\mathrm{CSTC}}}{\delta \mathcal{H}^{2}}\right\rangle\left\langle\frac{\delta^{2} \mathcal{L}_{U}^{\mathrm{CSTC}}}{\delta S^{2}}\right\rangle<0, \quad\left\langle\frac{\delta^{2} \mathcal{L}_{U}^{\mathrm{CSTC}}}{\delta \mathcal{H}^{2}}\right\rangle<0, \quad\left\langle\frac{\delta^{2} \mathcal{L}_{U}^{\mathrm{CSTC}}}{\delta S^{2}}\right\rangle<0,
$$

leading to

$$
\lambda_{\mathrm{TC}}>-\frac{m_{\tilde{\pi}}^{2}}{2 u^{2}}, \quad \lambda_{H}>0,
$$

which are automatically satisfied for the positively defined scalar mass form, i.e. for $M_{\tilde{\sigma}}^{2}>0$ and $M_{h}^{2}>0$.

Notice that in the limiting case of $\mu_{\mathrm{S}, \mathrm{H}} \ll m_{\tilde{\pi}}$ which, in principle, is not forbidden (while origin of $\mu$-terms is generally unclear in the SM theory) and even can be motivated in the nearly conformal limit of new strongly coupled dynamics (see below), both vevs $v$ and $u$ are expressed in terms of the technifermion condensate, having thereby the same dynamical origin. The extra confined TC sector is now responsible for the EWSB in the CSTC model, so the role of extra $\mu$-terms, which are usually required for the classical Higgs mechanism in the rigorous SM formulation, is taken over by the technifermion condensate. This observation thus supports the above argument about the common quantum-topological nature of the EWSB and the chiral symmetry breaking mechanisms in the considering CSTC model. In what follows, we discuss both cases. In the first case, for the sake of generality, we keep the scalar $\mu$-terms permitted by the gauge symmetry as free independent parameters. In the second theoretically motivated limiting case $\mu_{\mathrm{S}, \mathrm{H}} \ll m_{\tilde{\pi}}$, we will also consider the minimal CSTC model neglecting the small $\mu$-terms below. 
In the general case, the mass form of the scalar fields can be diagonalized and represented in the form

$$
\Delta \mathcal{L}_{s c}^{\mathrm{CSTC}}=-\frac{1}{2}\left[m_{\tilde{\pi}}^{2}\left(2 \tilde{\pi}^{+} \tilde{\pi}^{-}+\tilde{\pi}^{0} \tilde{\pi}^{0}\right)+M_{\tilde{\sigma}}^{2} \tilde{\sigma}^{2}+M_{h}^{2} h^{2}\right]
$$

where the technipion mass squared expressed in terms of vevs and scalar self-couplings is

$$
m_{\tilde{\pi}}^{2}=\lambda_{\mathrm{TC}} u^{2}-\lambda v^{2}-\mu_{\mathrm{S}}^{2},
$$

the technisigma and Higgs boson masses squared are

$$
\begin{aligned}
& M_{h}^{2}=\frac{1}{2}\left[2 \lambda_{\mathrm{TC}} u^{2}+m_{\tilde{\pi}}^{2}+2 \lambda_{\mathrm{H}} v^{2}-\sqrt{\left(2 \lambda_{\mathrm{TC}} u^{2}+m_{\tilde{\pi}}^{2}-2 \lambda_{\mathrm{H}} v^{2}\right)^{2}+16 \lambda^{2} u^{2} v^{2}}\right], \\
& M_{\tilde{\sigma}}^{2}=\frac{1}{2}\left[2 \lambda_{\mathrm{TC}} u^{2}+m_{\tilde{\pi}}^{2}+2 \lambda_{\mathrm{H}} v^{2}+\sqrt{\left(2 \lambda_{\mathrm{TC}} u^{2}+m_{\tilde{\pi}}^{2}-2 \lambda_{\mathrm{H}} v^{2}\right)^{2}+16 \lambda^{2} u^{2} v^{2}}\right],
\end{aligned}
$$

respectively. Finally, the expression for the $h \tilde{\sigma}$-mixing angle reads

$$
\tan 2 \theta=\frac{4 \lambda u v}{2 \lambda_{\mathrm{TC}} u^{2}+m_{\tilde{\pi}}^{2}-2 \lambda_{\mathrm{H}} v^{2}},
$$

whereas the sign of $s_{\theta}$ is given by

$$
\operatorname{sign}\left(s_{\theta}\right)=\operatorname{sign}\left(\frac{\lambda u v}{2 \lambda_{\mathrm{H}} v^{2}-M_{h}^{2}}\right) .
$$
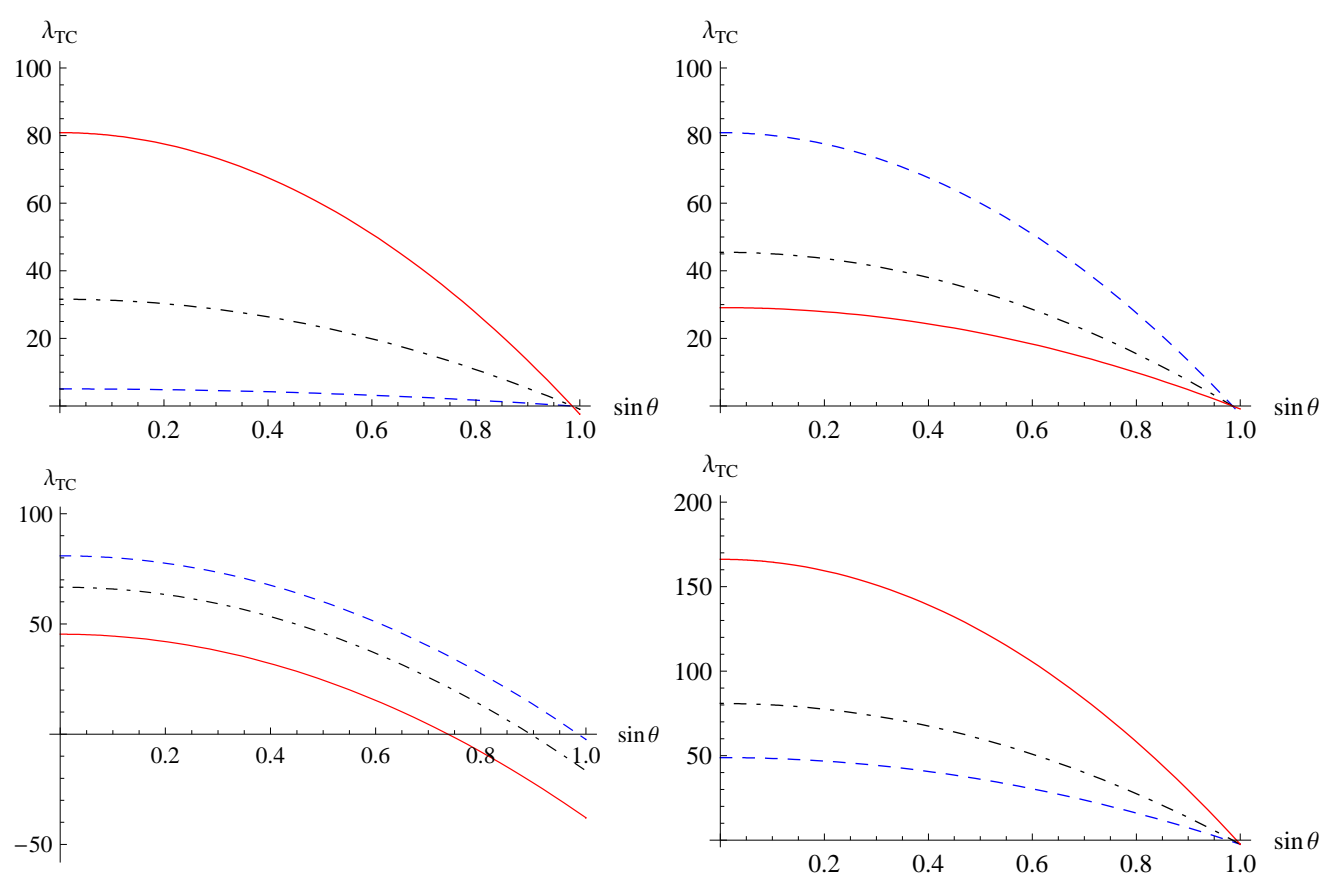

FIG. 3: Dependence of the quartic TC self-coupling $\lambda_{\mathrm{TC}}$ on the $h \tilde{\sigma}$-mixing $s_{\theta}$ with dashed, dashdotted and solid lines corresponding to $(1) g_{\mathrm{TC}}=2,5,8, M_{\tilde{Q}}=300 \mathrm{GeV}, m_{\tilde{\pi}}=150 \mathrm{GeV}$, and $M_{\tilde{\sigma}}=500 \mathrm{GeV} ;(2) g_{\mathrm{TC}}=8, M_{\tilde{Q}}=300,400,500 \mathrm{GeV}, m_{\tilde{\pi}}=150 \mathrm{GeV}$, and $M_{\tilde{\sigma}}=500 \mathrm{GeV} ;(3)$ $g_{\mathrm{TC}}=8, M_{\tilde{Q}}=300 \mathrm{GeV}, m_{\tilde{\pi}}=150,250,350 \mathrm{GeV}$, and $M_{\tilde{\sigma}}=500 \mathrm{GeV} ;(4) g_{\mathrm{TC}}=8, M_{\tilde{Q}}=300$ $\mathrm{GeV}, m_{\tilde{\pi}}=150 \mathrm{GeV}$, and $M_{\tilde{\sigma}}=400,500,700 \mathrm{GeV}$, in each plot from top to bottom and left to right, respectively. Here and below, $M_{h}=125 \mathrm{GeV}$. The coupling $\lambda_{\mathrm{TC}}$ is symmetric w.r.t. $s_{\theta} \rightarrow-s_{\theta}$. 

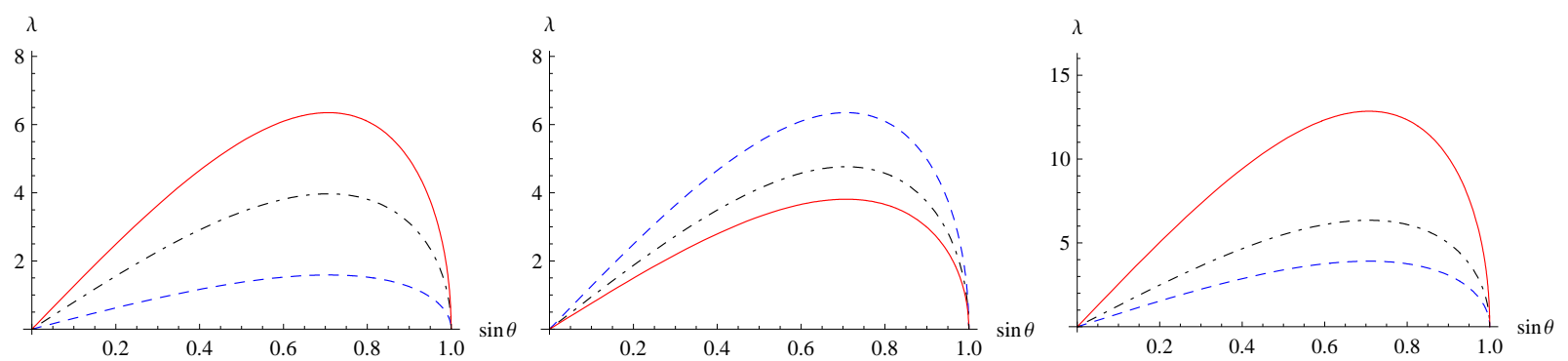

FIG. 4: Dependence of the quartic Higgs-TC coupling $\lambda$ on the $h \tilde{\sigma}$-mixing $s_{\theta}$ with dashed, dashdotted and solid lines corresponding to $(1) g_{\mathrm{TC}}=2,5,8, M_{\tilde{Q}}=300 \mathrm{GeV}$, and $M_{\tilde{\sigma}}=500 \mathrm{GeV}$; (2) $g_{\mathrm{TC}}=8, M_{\tilde{Q}}=300,400,500 \mathrm{GeV}$, and $M_{\tilde{\sigma}}=500 \mathrm{GeV} ;(3) g_{\mathrm{TC}}=8, M_{\tilde{Q}}=300 \mathrm{GeV}$, and $M_{\tilde{\sigma}}=400,500,700 \mathrm{GeV}$, in each plot from left to right, respectively. It does not depend on $m_{\tilde{\pi}}$. The coupling $\lambda$ is antisymmetric w.r.t. $s_{\theta} \rightarrow-s_{\theta}$.

In general, the additional sector of the Lagrangian under discussion together with the modified SM Higgs sector contains seven parameters in total, namely

$$
\mu_{\mathrm{H}}^{2}, \quad \mu_{\mathrm{S}}^{2}, \quad \lambda_{\mathrm{H}}, \quad \lambda_{\mathrm{TC}}, \quad \lambda, \quad g_{\mathrm{TC}}, \quad\langle\overline{\tilde{Q}} \tilde{Q}\rangle .
$$

In phenomenological studies, it can be convenient to turn to mathematically equivalent set of other independent physical parameters, namely,

$$
M_{h}, \quad M_{\tilde{\sigma}}, \quad m_{\tilde{\pi}}, \quad M_{W}, \quad M_{\tilde{Q}}, \quad g_{\mathrm{TC}}, \quad s_{\theta} .
$$

where $M_{\tilde{Q}}=g_{\mathrm{TC}} u$ is the constituent technifermion mass. For this purpose, the following relations between scalar self-couplings and physical quantities (2.20) following directly from Eqs. (2.15), (2.16) and (2.17) can be useful:

$$
\begin{gathered}
2 \lambda_{\mathrm{TC}} u^{2}=-m_{\tilde{\pi}}^{2}+M_{\tilde{\sigma}}^{2} c_{\theta}^{2}+M_{h}^{2} s_{\theta}^{2}, \\
2 \lambda_{\mathrm{H}} v^{2}=M_{\tilde{\sigma}}^{2} s_{\theta}^{2}+M_{h}^{2} c_{\theta}^{2}, \\
2 \lambda u v= \pm\left(M_{\tilde{\sigma}}^{2}-M_{h}^{2}\right) c_{\theta} s_{\theta} .
\end{gathered}
$$

In reality, two mass parameters in Eq. (2.20) can be fixed by the SM phenomenology, namely, $M_{W} \simeq 80.4 \mathrm{GeV}$ and $M_{h} \simeq 125.3 \mathrm{GeV}$, so effectively only five-dimensional parameter space remains to be analyzed. Apparently, two phenomenologically interesting cases are possible: the lightest observed scalar particle is indeed the Higgs boson, then $M_{h}<M_{\tilde{\sigma}}$, or the technisigma is the lightest one $M_{\tilde{\sigma}}<M_{h}$. In Eq. (2.21) we restrict ourselves to the first solution for $\lambda$, with "plus" sign, and fix $\cos \theta>0$, such that the sign of $\lambda$ is the same as the sign of $s_{\theta}$ for $M_{\tilde{\sigma}}>M_{h}$, opposite to the sign of $s_{\theta}$ for reversed hierarchy $M_{\tilde{\sigma}}<M_{h}$. In what follows, we work with the direct mass hierarchy with the lightest Higgs boson in the scalar sector of the model $M_{\tilde{\sigma}}>M_{h}$, unless noted otherwise.

In Fig. 3 we represent dependence of the quartic TC self-coupling $\lambda_{\mathrm{TC}}$ on the $h \tilde{\sigma}$-mixing angle, or more precisely $s_{\theta}$, over reasonable ranges of $g_{\mathrm{TC}}, M_{\tilde{Q}}, m_{\tilde{\pi}}$ and $M_{\tilde{\sigma}}$ parameters. One notices that $\lambda_{\mathrm{TC}}$ vanishes in the maximal $h \tilde{\sigma}$-mixing limit $s_{\theta} \rightarrow 1$ for any $g_{\mathrm{TC}}, M_{\tilde{Q}}, M_{\tilde{\sigma}}$ values and for small $m_{\tilde{\pi}} \sim 150 \mathrm{GeV}$. For small mixing angles and rather large $M_{\tilde{\sigma}} \gtrsim 700 \mathrm{GeV}$ 


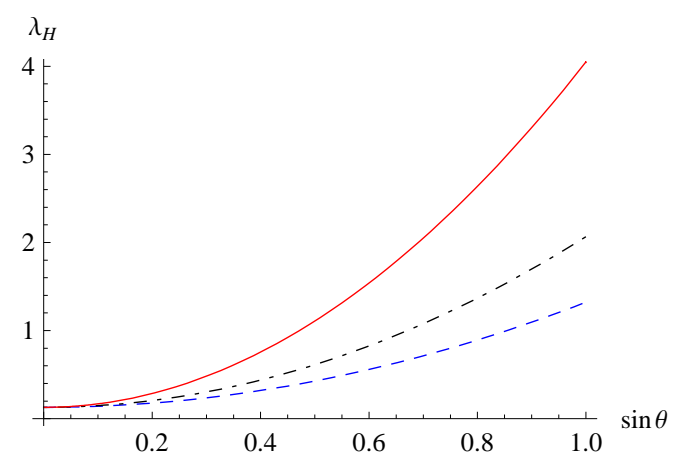

FIG. 5: Dependence of the quartic Higgs boson self-coupling $\lambda_{\mathrm{H}}$ on the $h \tilde{\sigma}$-mixing $s_{\theta}$ with dashed, dash-dotted and solid lines corresponding to $M_{\tilde{\sigma}}=400,500,700 \mathrm{GeV}$, respectively. It does not depend on other free parameters of the CSTC model. The coupling $\lambda_{\mathrm{H}}$ is symmetric w.r.t. $s_{\theta} \rightarrow-s_{\theta}$.

and $g_{\mathrm{TC}} \gtrsim 8$, it can become very large $\lambda_{\mathrm{TC}} \sim 100$, where the non-linear non-perturbative effects turn out to be important, and applicability of the corresponding GLT $\sigma \mathrm{M}$ may be restricted. This has to be taken into consideration in analysis of the available parameter space of the model and possible phenomenological signatures.

Similarly, the quartic Higgs-TC coupling $\lambda$ and the quartic Higgs boson self-coupling $\lambda_{\mathrm{H}}$ w.r.t. $s_{\theta}$ are given in Figs. 4 and 5 , respectively. The $\lambda$ coupling does not depend on the technipion mass, and vanish in both limits $s_{\theta} \rightarrow 1$ and $s_{\theta} \rightarrow 0$ limits as is seen in figures. The $\lambda_{H}$ coupling depends only on the $s_{\theta}$ and $M_{\tilde{\sigma}}$, and both $\lambda$ and $\lambda_{\mathrm{H}}$ are generally constrained $\lambda, \lambda_{\mathrm{H}} \lesssim 10$.

In our analysis for the sake of simplicity and transparency we wish to employ an analogy with QCD and hadron physics as long as possible, which is reasonable (even though not necessary) since the TC confinement group and technifermion hypercharge are assumed to be the same as for standard quarks. If such an analogy is indeed realized in nature, one would need to pay attention to other possible similarities e.g. in properties of QCD and techni-QCD vacuum subsystems. The QCD vacuum at scales $\Lambda_{\mathrm{QCD}} \sim 200 \mathrm{MeV}$ is formed by gluon and quark condensates [43]:

$$
\begin{aligned}
& \left\langle 0\left|\frac{\alpha_{s}}{\pi} \hat{G}_{\mu \nu} \hat{G}^{\mu \nu}\right| 0\right\rangle=(365 \pm 20 \mathrm{MeV})^{4} \simeq\left(2 \Lambda_{\mathrm{QCD}}\right)^{4}, \\
& \langle 0|\bar{u} u| 0\rangle=\langle 0|\bar{d} d| 0\rangle=-l_{g}\left\langle 0\left|\frac{\alpha_{s}}{\pi} \hat{G}_{\mu \nu} \hat{G}^{\mu \nu}\right| 0\right\rangle=-(235 \pm 15 \mathrm{MeV})^{3},
\end{aligned}
$$

where $\Lambda_{\mathrm{QCD}}^{-1} \simeq 10^{-13} \mathrm{sm}$ is the characteristic hadron size, whereas the correlation length $l_{g} \simeq(1500 \mathrm{MeV})^{-1}$ is the characteristic length scale of the non-perturbative gluon field fluctuations. In the meson spectrum, the lightest states are pions with mass $m_{\pi} \simeq 140 \mathrm{MeV}$ (the pseudo-Goldstone modes of the quark condensate excitations) and $\sigma$-meson $\sigma=f_{0}(500)$ with mass $m_{\sigma} \simeq 500 \mathrm{MeV}$ (the lightest glueball as a collective excitation of the gluon condensate). In the framework of the hypothesis about the technicolor nature of the Higgs vacuum $v \sim 200 \mathrm{GeV}$, it is natural to assume that the second techni-QCD vacuum subsystem is formed by condensate of technigluons and light technifermions at a nearby scale $\Lambda_{\mathrm{TC}} \gtrsim 200$ $\mathrm{GeV}$, being therefore, at least, a thousand times higher than $\Lambda_{\mathrm{QCD}}$ scale. Then, a reasonable 
order-of-magnitude estimate leads to

$$
\begin{aligned}
& \left\langle 0\left|\frac{\alpha_{\mathrm{TC}}}{\pi} \hat{F}_{\mu \nu} \hat{F}^{\mu \nu}\right| 0\right\rangle \sim\left(2 \Lambda_{\mathrm{TC}}\right)^{4}, \\
& \langle 0|\bar{U} U| 0\rangle=\langle 0|\bar{D} D| 0\rangle \sim-l_{\mathrm{TC}}\left(2 \Lambda_{\mathrm{TC}}\right)^{4} .
\end{aligned}
$$

If the current technifermion masses obey the same hierarchy as that of usual quarks, the lightest technihadron excitations in the bosonic spectrum are technipions $\tilde{\pi}^{0, \pm}$ and technisigma meson $\tilde{\sigma}$, whereas in the fermion spectrum - techninucleons $P$ and $N$. Such a dynamical similarity between color and technicolor enables us to estimate characteristic masses of the lightest technihadrons and constituent technifermions through the scale transformation of ordinary hadron states via scale factor $\zeta=\Lambda_{\mathrm{TC}} / \Lambda_{\mathrm{QCD}} \gtrsim 1000$, i.e.

$$
m_{\tilde{\pi}} \gtrsim 140 \mathrm{GeV}, \quad M_{\tilde{\sigma}} \gtrsim 500 \mathrm{GeV}, \quad M_{\tilde{Q}} \gtrsim 300 \mathrm{GeV}, \quad M_{P} \simeq M_{N} \gtrsim 1 \mathrm{TeV},
$$

which imply that $m_{\tilde{\pi}}>M_{h}, M_{\tilde{\sigma}}>M_{h}, M_{\tilde{\sigma}}>m_{\tilde{\pi}}$, and $u \gtrsim 100 \mathrm{GeV}$ for $g_{\mathrm{TC}} \simeq 3$. Also, with respect to interactions with known particles at typical 4-momentum squared transfers $Q^{2} \ll l_{\mathrm{TC}}^{-2} \gtrsim 2.3 \mathrm{TeV}^{2}$, the lightest technihadrons behave as elementary particles, which participate in electroweak interactions only. The technipions are then treated as being in the adjoint representation of the $S U(2)_{\mathrm{W}}$ with hypercharge equal to zero, thus justifying what was done above, whereas techninucleons can be included as the fundamental representation of the electroweak group $S U(2)_{\mathrm{W}} \otimes U(1)_{\mathrm{Y}}$ with hypercharge $Y_{\mathrm{TN}}=1$ along with the constituent technifermion doublet (2.2). Heavy techninucleons, however, are likely to be irrelevant for the LHC phenomenology, but can play an important role in astrophysics as a plausible candidate for the Dark Matter. The conditions (2.23) following from the analogy of QCD and techni-QCD will be used below in phenomenological studies in the CSTC framework.

\section{The physical Lagrangian of the CSTC model}

In this Section, we consider the principal part of the physical CSTC Lagrangian relevant for studies of the basic phenomenological processes in the CSTC model e.g. corrections to EW precision observables, as well as Higgs, technipion and technisigma production and decays, discussed below.

The vector-like interactions $\overline{\tilde{Q}} \tilde{Q} V$ of technifermions and gauge bosons $V=Z^{0}, W^{ \pm}, \gamma$ are given by

$$
\begin{aligned}
L_{\overline{\tilde{Q}} \tilde{Q} V} & =\frac{1}{\sqrt{2}} g \bar{U} \gamma^{\mu} D \cdot W_{\mu}^{+}+\frac{1}{\sqrt{2}} g \bar{D} \gamma^{\mu} U \cdot W_{\mu}^{-} \\
& +\frac{g}{c_{W}} Z_{\mu} \sum_{f=U, D} \bar{f} \gamma^{\mu}\left(t_{3}^{f}-q_{f} s_{W}^{2}\right) f+e \sum_{f=U, D} q_{f} \bar{f} \gamma^{\mu} A_{\mu} f,
\end{aligned}
$$

where $e=g s_{W}$ is the electron charge, $t_{3}^{f}$ is the weak isospin $\left(t_{3}^{U}=1 / 2, t_{3}^{D}=-1 / 2\right)$, $q_{f}=Y_{\tilde{Q}} / 2+t_{3}^{f}$ is the technifermion charge. As agreed above, we choose $Y_{\tilde{Q}}=1 / 3$ in analogy to the SM, thus $q_{U}=2 / 3$ and $q_{D}=-1 / 3$.

The Yukawa-type interactions $\overline{\tilde{Q}} \tilde{Q} h+\overline{\tilde{Q}} \tilde{Q} \tilde{\sigma}+\tilde{\bar{Q}} \tilde{Q} \tilde{\pi}$ of constituent technifermions with scalar $(h$ and $\tilde{\sigma})$ and pseudoscalar $\left(\pi^{0, \pm}\right)$ fields are driven by

$$
\begin{aligned}
& L_{\tilde{\tilde{Q}} \tilde{Q} h}+L_{\tilde{\tilde{Q}} \tilde{Q} \tilde{\sigma}}+L_{\tilde{\tilde{Q}} \tilde{Q} \tilde{\pi}}=-g_{\mathrm{TC}}\left(c_{\theta} \tilde{\sigma}+s_{\theta} h\right) \cdot(\bar{U} U+\bar{D} D) \\
& -i \sqrt{2} g_{\mathrm{TC}} \tilde{\pi}^{+} \bar{U} \gamma_{5} D-i \sqrt{2} g_{\mathrm{TC}} \tilde{\pi}^{-} \bar{D} \gamma_{5} U-i g_{\mathrm{TC}} \tilde{\pi}^{0}\left(\bar{U} \gamma_{5} U-\bar{D} \gamma_{5} D\right) .
\end{aligned}
$$


As was advocated above, at relatively low energies $\sim 0.1 \mathrm{TeV}$ close to the $M_{\mathrm{EW}}$ scale the Lagrangians of the technifermion interactions (2.24) and (2.25) should be used in the loopinduced processes with constituent quarks propagating inside loops only.

The interactions of technipions with gauge bosons which will be used in further calculations are defined as follows

$$
\begin{aligned}
L_{\tilde{\pi} \tilde{\pi} V} & =i g W^{\mu+} \cdot\left(\tilde{\pi}^{0} \tilde{\pi}_{, \mu}^{-}-\tilde{\pi}^{-} \tilde{\pi}_{, \mu}^{0}\right)+i g W^{\mu-} \cdot\left(\tilde{\pi}^{+} \tilde{\pi}_{, \mu}^{0}-\tilde{\pi}^{0} \tilde{\pi}_{, \mu}^{+}\right) \\
& +i g\left(c_{W} Z_{\mu}+s_{W} A_{\mu}\right) \cdot\left(\tilde{\pi}^{-} \tilde{\pi}_{, \mu}^{+}-\tilde{\pi}^{+} \tilde{\pi}_{, \mu}^{-}\right) \\
& +g^{2} W_{\mu}^{+} W^{\mu-} \cdot\left(\tilde{\pi}^{0} \tilde{\pi}^{0}+\tilde{\pi}^{+} \tilde{\pi}^{-}\right)+g^{2}\left(c_{W} Z_{\mu}+s_{W} A_{\mu}\right)^{2} \cdot \tilde{\pi}^{+} \tilde{\pi}^{-}+\ldots,
\end{aligned}
$$

where $\tilde{\pi}_{, \mu} \equiv \partial_{\mu} \tilde{\pi}$. All triple and quartic interactions, which are necessary in calculations of technipion contributions to the gauge bosons self-energies, are written down here.

The Yukawa interactions $\bar{f} f h+\bar{f} f \tilde{\sigma}$ of the ordinary fermions get modified compared to the SM

$$
L_{\bar{f} f h}+L_{\bar{f} f \tilde{\sigma}}=-g\left(c_{\theta} h-s_{\theta} \tilde{\sigma}\right) \cdot \frac{m_{f}}{2 M_{W}} \bar{f} f .
$$

The Lagrangians of the $h \tilde{\pi} \tilde{\pi}$ and $h W W+h Z Z$ interactions are

$$
\begin{gathered}
L_{h \tilde{\pi} \tilde{\pi}}=-\left(\lambda_{\mathrm{TC}} u s_{\theta}-\lambda v c_{\theta}\right) h\left(\tilde{\pi}^{0} \tilde{\pi}^{0}+2 \tilde{\pi}^{+} \tilde{\pi}^{-}\right)=-\frac{M_{h}^{2}-m_{\tilde{\pi}}^{2}}{2 M_{\tilde{Q}}} g_{\mathrm{TC}} s_{\theta} h\left(\tilde{\pi}^{0} \tilde{\pi}^{0}+2 \tilde{\pi}^{+} \tilde{\pi}^{-}\right), \\
L_{h W W}+L_{h Z Z}=g M_{W} c_{\theta} h W_{\mu}^{+} W^{\mu-}+\frac{1}{2}\left(g^{2}+g_{1}^{2}\right)^{1 / 2} M_{Z} c_{\theta} h Z_{\mu} Z^{\mu} .
\end{gathered}
$$

The Lagrangians of the $\tilde{\sigma} \tilde{\pi} \tilde{\pi}$ and $\tilde{\sigma} W W+\tilde{\sigma} Z Z$ interactions are

$$
\begin{gathered}
L_{\tilde{\sigma} \tilde{\pi} \tilde{\pi}}=-\left(\lambda_{\mathrm{TC}} u c_{\theta}+\lambda v s_{\theta}\right) \tilde{\sigma}\left(\tilde{\pi}^{0} \tilde{\pi}^{0}+2 \tilde{\pi}^{+} \tilde{\pi}^{-}\right)=-\frac{M_{\tilde{\sigma}}^{2}-m_{\tilde{\pi}}^{2}}{2 M_{\tilde{Q}}} g_{\mathrm{TC}} c_{\theta} \tilde{\sigma}\left(\tilde{\pi}^{0} \tilde{\pi}^{0}+2 \tilde{\pi}^{+} \tilde{\pi}^{-}\right), \\
L_{\tilde{\sigma} W W}+L_{\tilde{\sigma} Z Z}=-g M_{W} s_{\theta} \tilde{\sigma} W_{\mu}^{+} W^{\mu-}-\frac{1}{2}\left(g^{2}+g_{1}^{2}\right)^{1 / 2} M_{Z} s_{\theta} \tilde{\sigma} Z_{\mu} Z^{\mu} .
\end{gathered}
$$

The Lagrangian of quartic scalar-gauge $(\tilde{\sigma} / h)^{2} V V$ interactions is given by

$$
L_{(\tilde{\sigma} / h)^{2} V V}=\frac{1}{4}\left(c_{\theta} h-s_{\theta} \tilde{\sigma}\right)^{2} \cdot\left(g^{2} W_{\mu}^{+} W^{\mu-}+\frac{1}{2}\left(g^{2}+g_{1}^{2}\right) Z^{\mu} Z_{\mu}\right) .
$$

\section{E. Nearly conformal limit: the minimal CSTC}

In the SM, the arbitrary quadratic terms with "wrong" sign in the Higgs potential are usually required for the classical (non-quantum) Higgs mechanism of the EWSB. As we have noticed above, in the framework of the CSTC model there is a possibility for another interpretation of the Higgs mechanism in which the nature of all energy scales (including the Higgs vev) is quantum-topological. Let us look into the latter possibility in detail.

In the rigorous QCD framework, there is not any fundamental scalar sector and thus scalar $\mu$-terms do not appear. In the theory of non-perturbative QCD vacuum all the scale parameters have quantum-topological nature and are expressed through the gluon

condensate $\langle G G\rangle$ and the correlation length $l_{g}$, whereas the quark condensate $\langle q \bar{q}\rangle$ is induced 
by the gluon one (2.22). Clearly, low energy hadron physics based upon the effective GL $\sigma \mathrm{M}$ should reproduce the non-perturbative QCD predictions. On the other hand, it is wellknown that in the limit of small current quark masses $m_{q} \rightarrow 0$ (the chiral limit), the QCD Lagrangian restores the conformal symmetry. Similarly, the $\sigma$-model as an effective model of non-perturbative QCD should obey the conformal symmetry in the chiral QCD limit. In this case, the $\mu_{S}$-term corresponding to the $\sigma$ field is forbidden by the conformal symmetry. In a realistic case, the conformal symmetry in QCD is broken due to non-zeroth current quark masses. However, the current up- and down-quark masses are small compared to the value of the quark condensate $\langle q \bar{q}\rangle$ or, equivalently, the pion mass, i.e. $m_{u, d} \ll m_{\pi}$, so it is meaningful to assume that an induced $\mu_{S}$-term, if exists, should also be small $\mu_{S} \ll m_{\pi}$. In this case, since $\langle G G\rangle,\langle q \bar{q}\rangle$ and small current masses $m_{u, d} \ll m_{\pi}$ are the only physical parameters in non-perturbative QCD, the $\sigma$ vev $u \sim m_{\pi}$ has quantum-topological nature, so it should be expressed only through these parameters and given by e.g. $\langle q \bar{q}\rangle$ or, equivalently, $m_{\pi}$. Of course, this logic is rather naive since the $\sigma$-model does not have status of a fundamental theory, but rather serves as an effective low-energy phenomenological model with its own limitations and constraints. Note, a dynamical theory of the QCD vacuum does not exists yet, and our understanding of non-perturbative effects is very limited and one cannot make any strong claims here.

The above line of naive arguments can be naturally extended to the technifermion sector in confinement adopting a direct analogy between non-perturbative QCD and techniQCD. Looking at the Eqs. (2.9) we notice that for not very large scalar self-couplings $|\lambda|,\left|\lambda_{\mathrm{TC}}\right|, \lambda_{\mathrm{H}} \sim 0.1-10$ in the potential (2.8), the technisigma vev $u$ can be expressed through the technifermion condensate, or $m_{\tilde{\pi}}$, for small $\mu_{S} \ll m_{\tilde{\pi}}$ which can be valid in the nearly conformal limit of chiral techni-QCD $m_{U, D} \ll m_{\tilde{\pi}}$ if and only if the Higgs boson vev is also small compared to the techni-confinement scale, i.e. $\mu_{\mathrm{H}} \ll m_{\tilde{\pi}}$. The latter means that both the vacua, the Higgs and technisigma vevs, have the same quantum-topological nature and completely determined by the technifermion condensate. This theoretically appealing scenario would be rigorous and strictly valid in the exact chiral techni-QCD limit with vanishing current technifermion masses $m_{U, D} \rightarrow 0$. In the nearly-conformal limit there is a weak or no running of the strong techni-QCD coupling. This is in accordance with the analytic QCD (see e.g. Ref. [42]) or other phenomenological approaches predicting a rather slow bounded or even "frozen" behavior of the strong QCD coupling in the infrared domain while non-perturbative QCD contributions are strongly dominated over the perturbative ones in the constituent quark-meson interactions at small $Q^{2}$. To this end, in the nearly-conformal limit all the $\mu$-terms can be neglected in the Lagrangian (2.8) without affecting the SM Higgs mechanism itself, which then would be triggered completely by the technifermion condensate, giving rise to even more restricted parameter space of the model. Let us look into this non-trivial possibility, which is simply a particular case of the more general CSTC model described above, in some more detail.

The solutions of the two tadpole equations (2.10) can then be written w.r.t vevs as follows

$$
u=\left(\frac{\lambda_{\mathrm{H}}}{\delta}\right)^{1 / 3} \bar{g}_{\mathrm{TC}}^{1 / 3}, \quad v=\left(\frac{\xi \lambda}{\lambda_{\mathrm{H}}}\right)^{1 / 2}\left(\frac{\lambda_{\mathrm{H}}}{\delta}\right)^{1 / 3} \bar{g}_{\mathrm{TC}}^{1 / 3},
$$

where $\delta=\lambda_{\mathrm{H}} \lambda_{\mathrm{TC}}-\lambda^{2}, \bar{g}_{\mathrm{TC}}=g_{\mathrm{TC}}|\langle\tilde{\tilde{Q}} \tilde{Q}\rangle|>0$ and the $\operatorname{sign}$ factor $\xi=\operatorname{sign}\left(M_{\tilde{\sigma}}^{2}-3 m_{\tilde{\pi}}^{2}\right)$ such that $\xi \lambda \equiv|\lambda| \geq 0$ and $\lambda_{H}>0$ always. From relations (2.31) it follows that both vevs (and hence both the EWSB and the chiral symmetry breaking) are induced by the technifermion condensate since $u, v \sim|\langle\bar{Q} \tilde{Q}\rangle|^{1 / 3}$. So, our choice of the potential part of the TC Lagrangian 
$L_{U}(2.8)$ provides physically interesting interpretation of the Higgs vacuum condensate as triggered by the technifermion condensate $\langle\tilde{Q} \tilde{Q}\rangle \neq 0$ at low scales $\sim 0.1 \mathrm{TeV}$.

It is convenient to redefine yet unknown parameters, the technisigma vev, $u$, and $\bar{g}_{\mathrm{TC}}$ in terms of the Higgs vev, $v$, and scalar self-couplings $\lambda, \lambda_{\mathrm{H}}, \lambda_{\mathrm{TC}}$ as follows

$$
u=v \cdot\left(\frac{\lambda_{\mathrm{H}}}{\xi \lambda}\right)^{1 / 2}, \quad \bar{g}_{\mathrm{TC}}=v^{3}\left(\frac{\lambda_{\mathrm{H}} \lambda_{\mathrm{TC}}}{\lambda}-\lambda\right) \cdot\left(\frac{\lambda_{\mathrm{H}}}{\xi \lambda}\right)^{1 / 2} .
$$

The technipion mass is given by

$$
m_{\tilde{\pi}}^{2}=v^{2}\left(\frac{\lambda_{H} \lambda_{\mathrm{TC}}}{\lambda}-\lambda\right), \quad m_{\tilde{\pi}} \sim v .
$$

Note, in the limit $M_{\tilde{\sigma}} \rightarrow \sqrt{3} m_{\tilde{\pi}}$, we have $\delta \sim \lambda \rightarrow 0$, whereas $\bar{g}_{\mathrm{TC}} \sim u \sim M_{\tilde{Q}} \sim 1 / \sqrt{|\lambda|} \rightarrow \infty$ at finite $m_{\tilde{\pi}}$ and $v$. Also, $s_{\theta} \rightarrow 0$ in this case, so $h$ and $\tilde{\sigma}$ do not mix ("no $h \tilde{\sigma}$-mixing" limit). This peculiar limit physically corresponds to decoupling of the technifermion condensate (and hence the techniconfinement scale $\Lambda_{\mathrm{TC}}$ ) up to very high scales, while providing light technipions and technisigma in the spectrum and the TC-induced EWSB mechanism in the usual way. Of course, the formal mathematical singularities corresponding to a very large techniconfinement scale $\Lambda_{\mathrm{TC}}$, or equivalently, large $u$ and $|\langle\tilde{Q} \tilde{Q}\rangle|$ (see Fig. 7 below), should be regularized by yet unknown high-scale TC physics, and thus vicinities of these special points are to be excluded from the current consideration. Interestingly enough, the Higgs boson turns out to be absolutely standard close to the singular points - its properties are not affected by the extra TC degrees of freedom, since corresponding new TC-induced couplings vanish in this case at $M_{\tilde{\sigma}} \rightarrow \sqrt{3} m_{\tilde{\pi}}$. While physically possible, this peculiar situation, however, is not realized if one adopts the naive scaling between the QCD and techni-QCD considered in this analysis. Absence of any deviations from the SM in the measured Higgs boson properties, from the point of view of the minimal CSTC discussed here, would then mean physically that the "no $h \tilde{\sigma}$-mixing" scenario is realized in Nature, but this does not rule out the TC-induced EWSB mechanism (see below).

The mass form of the physical scalars, $h$ and $\tilde{\sigma}$ fields, can be represented by the following matrix

$$
M_{h \tilde{\sigma}}=\left(\begin{array}{cc}
3 m_{\tilde{\pi}}^{2}+2 \lambda v^{2} & -2 v^{2} \sqrt{\xi \lambda \lambda_{H}} \\
-2 v^{2} \sqrt{\xi \lambda \lambda_{H}} & 2 \lambda_{H} v^{2}
\end{array}\right) .
$$

The diagonalization of this matrix leads to masses of the physical states scalar states, i.e.

$$
M_{\tilde{\sigma}, h}^{2}=\frac{1}{2} v^{2}\left\{\left(2 \lambda_{H}+2 \lambda+3 \frac{m_{\tilde{\pi}}^{2}}{v^{2}}\right) \pm \sqrt{\left(2 \lambda_{H}+2 \lambda+3 \frac{m_{\tilde{\pi}}^{2}}{v^{2}}\right)^{2}+16 \lambda \lambda_{H}}\right\}
$$

Then, the $h \tilde{\sigma}$-mixing angle is given by

$$
c_{\theta}=\left(1+\frac{\left(M_{\tilde{\sigma}}^{2}-m_{11}\right)^{2}}{m_{12}^{2}}\right)^{-1 / 2}, \quad s_{\theta}=\xi \sqrt{1-c_{\theta}^{2}},
$$

where $m_{11}=\left(M_{h \tilde{\sigma}}\right)_{11}, m_{12}=\left(M_{h \tilde{\sigma}}\right)_{12}$ are the elements of the mass matrix (2.34). In analysis of the parameter space it is again convenient to express free scalar self-couplings 
$\left\{\lambda, \lambda_{\mathrm{H}}, \lambda_{\mathrm{TC}}\right\}$ through the physical masses $\left\{m_{\tilde{\pi}}^{2}, M_{\tilde{\sigma}}^{2}, M_{h}^{2}\right\}$ :

$$
\lambda=\frac{3 m_{\tilde{\pi}}^{2}\left(M_{\tilde{\sigma}}^{2}+M_{h}^{2}\right)-M_{\tilde{\sigma}}^{2} M_{h}^{2}-9 m_{\tilde{\pi}}^{4}}{6 v^{2} m_{\tilde{\pi}}^{2}}, \quad \lambda_{\mathrm{H}}=\frac{M_{\tilde{\tilde{\sigma}}}^{2} M_{h}^{2}}{6 v^{2} m_{\tilde{\pi}}^{2}}, \quad \lambda_{\mathrm{TC}}=\frac{\lambda}{\lambda_{\mathrm{H}}}\left(\lambda+\frac{m_{\tilde{\pi}}^{2}}{v^{2}}\right) .
$$

By fixing the Higgs boson mass to its recently measured value $M_{h} \simeq 125 \mathrm{GeV}$, one further reduces the freedom down to three free parameters only, $\left\{m_{\tilde{\pi}}, M_{\tilde{\sigma}}, M_{\tilde{Q}}\right\}$, compared to five parameters in the non-minimal case (cf. Section II.C). Note, the scalar self-couplings and the mixing angle $\theta$ depend only on two parameters $\left\{m_{\tilde{\pi}}, M_{\tilde{\sigma}}\right\}$, whereas $M_{\tilde{Q}}$ can be used to define $g_{\mathrm{TC}}$ or $\langle\bar{Q} \tilde{Q}\rangle$.

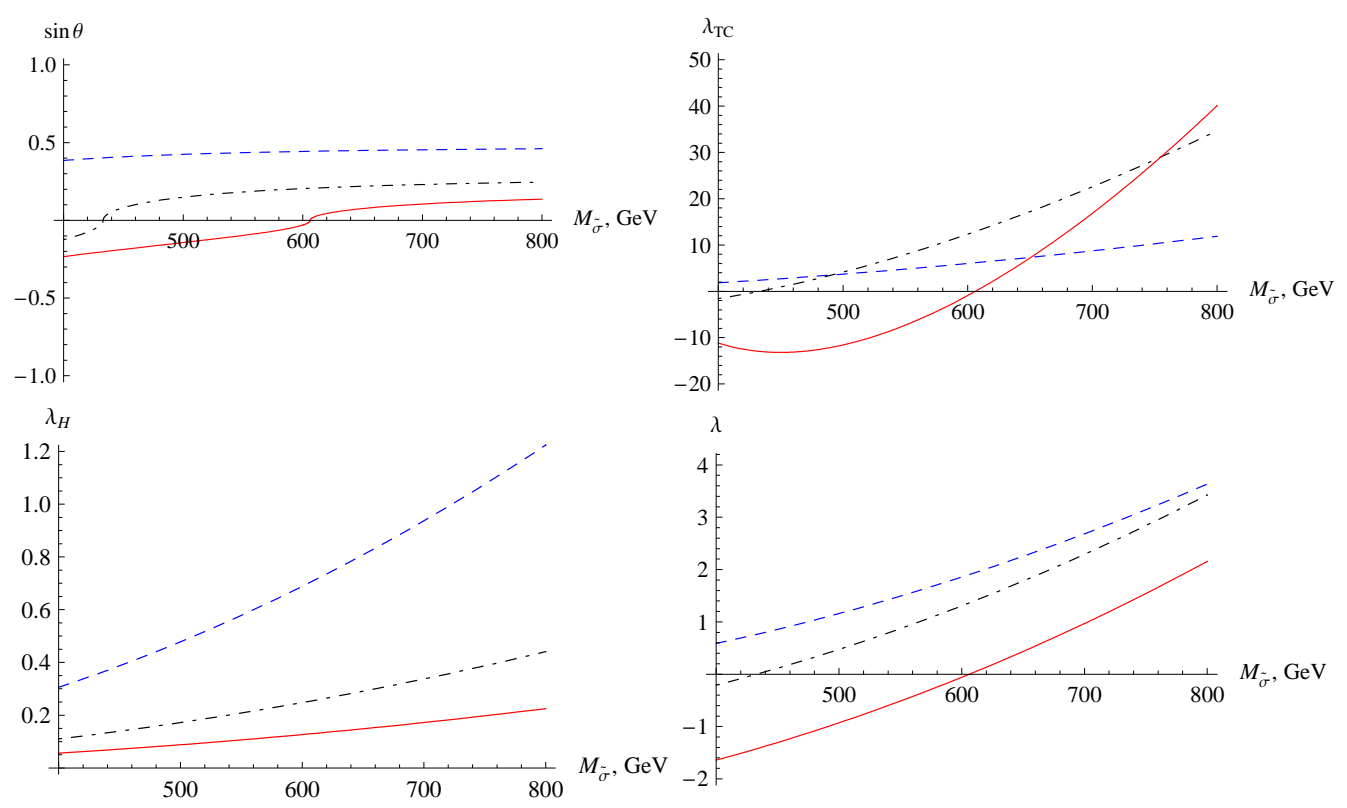

FIG. 6: Dependence of the $h \tilde{\sigma}$-mixing $s_{\theta}$ and the Higgs/TC self-couplings, $\lambda_{\mathrm{TC}}, \lambda_{\mathrm{H}}, \lambda$, on $M_{\tilde{Q}}$ in the minimal CSTC scenario with dashed, dash-dotted and solid lines corresponding to $m_{\tilde{\pi}}=$ $150,250,350 \mathrm{GeV}$, respectively. The "no $h \tilde{\sigma}$-mixing" limit corresponds to zeros of the curves at $M_{\tilde{\sigma}}=\sqrt{3} m_{\tilde{\pi}}$.

In Fig. [6 we have presented plots of sine of the mixing angle $s_{\theta}=s_{\theta}\left(m_{\tilde{\pi}}, m_{\tilde{\sigma}}\right)$, and scalar self-couplings - Higgs-(pseudo)scalar coupling $\lambda=\lambda\left(m_{\tilde{\pi}}, m_{\tilde{\sigma}}\right)$, quartic Higgs self-coupling $\lambda_{\mathrm{H}}=\lambda_{\mathrm{H}}\left(m_{\tilde{\pi}}, m_{\tilde{\sigma}}\right)$ and (pseudo)scalar self coupling $\lambda_{\mathrm{TC}}=\lambda_{\mathrm{TC}}\left(m_{\tilde{\pi}}, m_{\tilde{\sigma}}\right)$. At relatively large technipion masses $m_{\tilde{\pi}} \gtrsim 250 \mathrm{GeV}$ the $h \tilde{\sigma}$-mixing becomes rather small, $s_{\theta} \lesssim 0.2$, while it does not strongly depend on the technisigma mass, away from "no-mixing" points. As was noticed above, the condition $\lambda=0$ (or $s_{\theta}=0$ ) corresponds to "no-mixing" limit and is represented by a relation on masses, $M_{\tilde{\sigma}}=\sqrt{3} m_{\tilde{\pi}}$. In the considering ranges of masses, the values of $\lambda$ and $\lambda_{\mathrm{H}}$ do not exceed a few units, so they are of the order of strong ("fat") couplings in usual hadron dynamics (e.g. $g_{\rho \pi \pi} \sim 5-6$ ) and gradually increase at large $M_{\tilde{\sigma}}$. The (pseudo)scalar self-coupling $\lambda_{\mathrm{TC}}$ can reach larger values $\sim 100$ at large values of $M_{\tilde{\sigma}} \gtrsim 800 \mathrm{GeV}$ restricting the allowable region of physical parameters and applicability of the GLT $\sigma \mathrm{M}$ under consideration. An experimental information on the scalar self-couplings $\lambda, \lambda_{\mathrm{TC}}$ would shed light on the true origin of the Higgs mechanism making it possible to determined which minimal or non-minimal CSTC scenario is realized in Nature. 

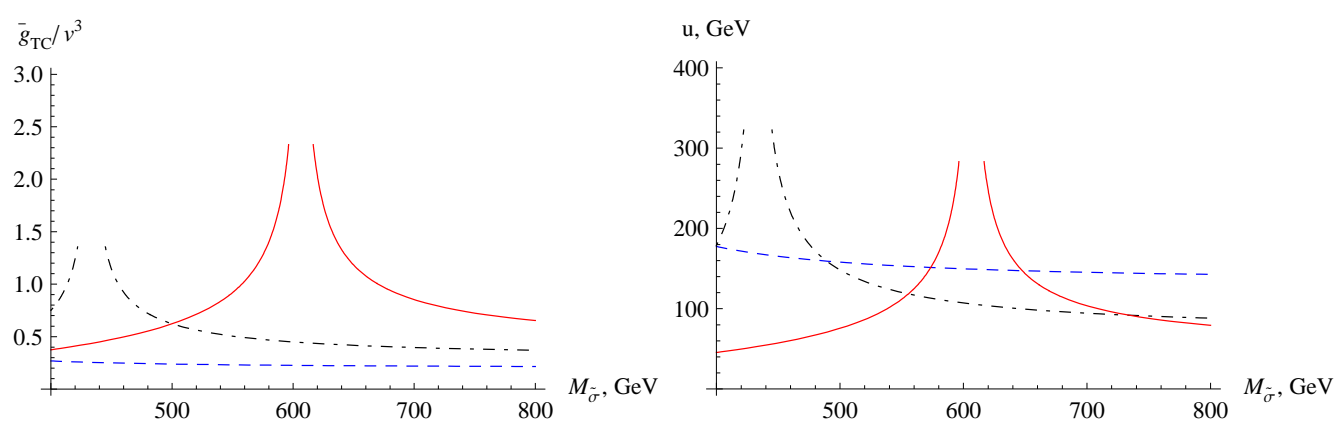

FIG. 7: The dimensionless $\bar{g}_{\mathrm{TC}} / v^{3}$ (left) and $\tilde{\sigma}$ vev $u$ (right) parameters with respect to $M_{\tilde{\sigma}}$ in the minimal CSTC scenario with dashed, dash-dotted and solid lines corresponding to $m_{\tilde{\pi}}=150,250,350 \mathrm{GeV}$, respectively. The "no $h \tilde{\sigma}$-mixing" limit corresponds to positions of the singularities on the curves at $M_{\tilde{\sigma}}=\sqrt{3} m_{\tilde{\pi}}$, vicinity of those are excluded from the plots.

In Fig. 7 we show the dimensionless $\bar{g}_{\mathrm{TC}} / v^{3}$ (left) and $\tilde{\sigma}$ vev $u$ (right) parameters with respect to $M_{\tilde{\sigma}}$ for different values of $m_{\tilde{\pi}}$ in the minimal CSTC scenario. The technisigma vev $u=u\left(m_{\tilde{\pi}}, m_{\tilde{\sigma}}\right)$, can be smaller than the Higgs vev $v, u \lesssim v$, almost in all physically favorable regions of parameter space where $m_{\tilde{\pi}} \sim v$, except for vicinities of "no $h \tilde{\sigma}$-mixing" points $M_{\tilde{\sigma}} \simeq \sqrt{3} m_{\tilde{\pi}}$ where $u$ can be larger or even much larger than the Higgs vev $v$. The latter case can be interesting both theoretically and phenomenologically in case of absence of any deviations of Higgs boson properties from the SM predictions at the LHC. Then, the only source of new information about the TC sector can only come from measurements of the Higgs boson scalar self-couplings and possible technipion/technisigma phenomenology.

One should notice here that if the small $h \tilde{\sigma}$-mixing scenario with $s_{\theta} \rightarrow 0$ and $\lambda \rightarrow 0$ is realized in Nature, we have the Technicolor decoupling regime with large $u \gg v$ and hence $\Lambda_{\mathrm{TC}} \sim M_{\tilde{Q}} \gg M_{\mathrm{EW}}$, while the Higgs boson, technipions and technisigma remain at the EW scale according to the tree-level mass formulae of the model. Remarkably enough, the Higgs vev is still expressed in terms of the technifermion condensate by Eq. (2.31) for vanishingly small but non-zeroth $\lambda \neq 0$ preserving the dynamical nature of the EWSB (or effective Higgs) mechanism.

\section{ELECTRO-WEAK CONSTRAINTS ON THE CSTC}

\section{A. Oblique corrections}

The effects of heavy New Physics (NP) particles of various types onto $Z^{0}$ and $W^{ \pm}$observables (e.g. masses, widths) typically emerging through extra loop contributions to $Z^{0}$, $W^{ \pm}$and $\gamma$ (diagonal and non-diagonal) self-energies can be parameterized by means of the so-called oblique corrections or Peskin-Takeuchi (PT) parameters [15]. The first three of these parameters $S, T, U$ are normally introduced in the limiting case of large masses of new particles compared to the EW scale, i.e. in the limit $M_{\mathrm{EW}} / M_{\mathrm{NP}} \ll 1\left(M_{\mathrm{NP}}\right.$ is the NP scale). If one relaxes this assumption, the $S, T, U$ parameters get somewhat modified, and additional three independent parameters denoted as $V, W, X$ are introduced (see e.g. Refs. [44, 45]). The oblique corrections are rather strongly constrained by the EW precision measurements [46]

$$
S=0.00_{-0.10}^{+0.11}, \quad T=0.02_{-0.12}^{+0.11}, \quad U=0.08 \pm 0.11
$$


and must be respected by realistic NP models (for existing constraints on higher $V, W, X$ parameters see e.g. Ref. [47]). The extensive studies of these constraints are very critical for all existing TC models. In particular, some of the traditional TC scenarios are currently being ruled out or are in a considerable tension with constraints on $S, T, U$ parameters [15] (see also Refs. [16, 17]). Let us analyze these constraints in the suggested CSTC scenario.

The analysis we present further in this Section does not depend on whether one includes $\mu_{S, H}$-terms or not; the difference between these non-minimal and minimal versions of the CSTC model can only be crucial for processes with (pseudo)-scalar self-couplings, which can be important e.g. for Higgs and technipion phenomenology.

In the earlier Sections, we have established the phenomenologically reasonable intervals for masses and couplings of new TC particles (technipions, technisigma and constituent technifermions) based on analogies with ordinary QCD and hadron physics together with the relative proximity of the new TC scale $\Lambda_{\mathrm{TC}} \sim 0.1-1 \mathrm{TeV}$. In what follows, these regions of parameter space will be tested against the EW precision constraints given by Eq. (3.1).

The generic definitions of the PT parameters are given in terms of corrections to the vacuum polarization functions $\delta \Pi_{\mathrm{XY}}\left(q^{2}\right)$ of the gauge bosons $(\mathrm{X}, \mathrm{Y}=\mathrm{W}, \mathrm{Z}, \gamma)$ coming either from new particles, additional to those in the $\operatorname{SM}, \Pi_{\mathrm{XY}}^{\text {new }}\left(q^{2}\right)$, or from a modification of the SM parameters due to NP effects, $\Pi_{\mathrm{XY}}^{\mathrm{SM}^{\prime}}\left(q^{2}\right)$, i.e.

$$
\delta \Pi_{\mathrm{XY}}\left(q^{2}\right) \equiv \Pi_{\mathrm{XY}}^{\mathrm{NP}}\left(q^{2}\right)-\Pi_{\mathrm{XY}}^{\mathrm{SM}}\left(q^{2}\right), \quad \Pi_{\mathrm{XY}}^{\mathrm{NP}}\left(q^{2}\right)=\Pi_{\mathrm{XY}}^{\mathrm{new}}\left(q^{2}\right)+\Pi_{\mathrm{XY}}^{\mathrm{SM}^{\prime}}\left(q^{2}\right) .
$$

The expressions for the $S, T, U$ parameters in terms of generic polarisation functions $\delta \Pi_{\mathrm{XY}}\left(q^{2}\right)$ and their derivatives $\delta \Pi_{\mathrm{XY}}^{\prime}\left(q^{2}\right)=d \delta \Pi / d q^{2}$ calculated beyond the linear approximation in $q^{2}$ variable read [44, 45]

$$
\begin{aligned}
& \frac{\alpha}{4 s_{W}^{2} c_{W}^{2}} S=\frac{\delta \Pi_{Z Z}\left(M_{Z}^{2}\right)-\delta \Pi_{Z Z}(0)}{M_{Z}^{2}}-\frac{c_{W}^{2}-s_{W}^{2}}{c_{W} s_{W}} \delta \Pi_{Z \gamma}^{\prime}(0)-\delta \Pi_{\gamma \gamma}^{\prime}(0), \\
& \alpha T=\frac{\delta \Pi_{W W}(0)}{M_{W}^{2}}-\frac{\delta \Pi_{Z Z}(0)}{M_{Z}^{2}}, \\
& \frac{\alpha}{4 s_{W}^{2}} U=\frac{\delta \Pi_{W W}\left(M_{W}^{2}\right)-\delta \Pi_{W W}(0)}{M_{W}^{2}}-c_{W}^{2} \frac{\delta \Pi_{Z Z}\left(M_{Z}^{2}\right)-\delta \Pi_{Z Z}(0)}{M_{Z}^{2}} \\
&-s_{W}^{2} \delta \Pi_{\gamma \gamma}^{\prime}(0)-2 c_{W} s_{W} \delta \Pi_{Z \gamma}^{\prime}(0) .
\end{aligned}
$$

Note, in the limit $\zeta=M_{\mathrm{EW}} / \Lambda_{\mathrm{TC}} \ll 1$ we have

$$
\begin{aligned}
\frac{\delta \Pi_{W W}\left(M_{Z}^{2}\right)-\delta \Pi_{W W}(0)}{M_{Z}^{2}} & =\frac{\delta \Pi_{W W}\left(M_{W}^{2}\right)-\delta \Pi_{W W}(0)}{M_{W}^{2}}+\mathcal{O}\left(M_{\mathrm{EW}}^{4} / \Lambda_{\mathrm{TC}}^{4}\right), \\
\frac{\delta \Pi_{\mathrm{XY}}\left(q^{2}\right)-\delta \Pi_{\mathrm{XY}}(0)}{q^{2}} & =\delta \Pi_{\mathrm{XY}}^{\prime}(0)+\mathcal{O}\left(q^{4} / \Lambda_{\mathrm{TC}}^{4}\right),
\end{aligned}
$$

which are equivalent to working in the linear order in $q^{2}$ in power expansions of the polarisation functions $\delta \Pi_{\mathrm{XY}}\left(q^{2}\right)$. In fact, applying approximate relation (3.5) to expressions (3.3) at $q^{2}=M_{Z}^{2}$ and having in mind that $\delta \Pi_{Z \gamma}(0)=\delta \Pi_{\gamma \gamma}(0)=0$ in a realistic case, one arrives at the Particle Data Group formulas (see Eq. (10.65b,c) in Ref. [46]). We, however, do not assume smallness of $\zeta$ in calculations (unless noted otherwise) since the new TC scale $\Lambda_{\mathrm{TC}}$ can be rather close to the electroweak scale $M_{\mathrm{EW}}$ since they may have the same physical nature in the considering CSTC scenario, and therefore rigorous definitions (3.3) should be applied. 
Other three parameters which appear beyond the linear order in $q^{2}$ in addition to the $S, T, U$ are defined as follows [44, 45]

$$
\begin{aligned}
\alpha V & =\delta \Pi_{Z Z}^{\prime}\left(M_{Z}^{2}\right)-\frac{\delta \Pi_{Z Z}\left(M_{Z}^{2}\right)-\delta \Pi_{Z Z}(0)}{M_{Z}^{2}} \\
\alpha W & =\delta \Pi_{W W}^{\prime}\left(M_{W}^{2}\right)-\frac{\delta \Pi_{W W}\left(M_{W}^{2}\right)-\delta \Pi_{W W}(0)}{M_{W}^{2}} \\
\alpha X & =-s_{W} c_{W}\left[\frac{\delta \Pi_{Z \gamma}\left(M_{Z}^{2}\right)}{M_{Z}^{2}}-\delta \Pi_{Z \gamma}^{\prime}(0)\right] .
\end{aligned}
$$

In the framework of the CSTC model, the new contributions to $W, Z$ and $\gamma$ vacuum polarizations come from technipion, constituent technifermions and technisigma loops, i.e.

$$
\Pi_{\mathrm{XY}}^{\text {new }}\left(q^{2}\right)=\Pi_{\mathrm{XY}}^{\tilde{\pi}}\left(q^{2}\right)+\Pi_{\mathrm{XY}}^{\tilde{Q}}\left(q^{2}\right)+\Pi_{\mathrm{XY}}^{\tilde{\sigma}}\left(q^{2}\right)
$$

while the SM modified contributions come only from the Higgs boson due to modified $h V V$ couplings, $\Pi_{\mathrm{XY}}^{h}\left(q^{2}\right)$, whereas other SM couplings are not changed in the CSTC model, thus we have

$$
\Pi_{\mathrm{XY}}^{\mathrm{SM}^{\prime}}\left(q^{2}\right)=\Pi_{\mathrm{XY}}^{h}\left(q^{2}\right)
$$

The corresponding diagrams are presented in Fig. 8.
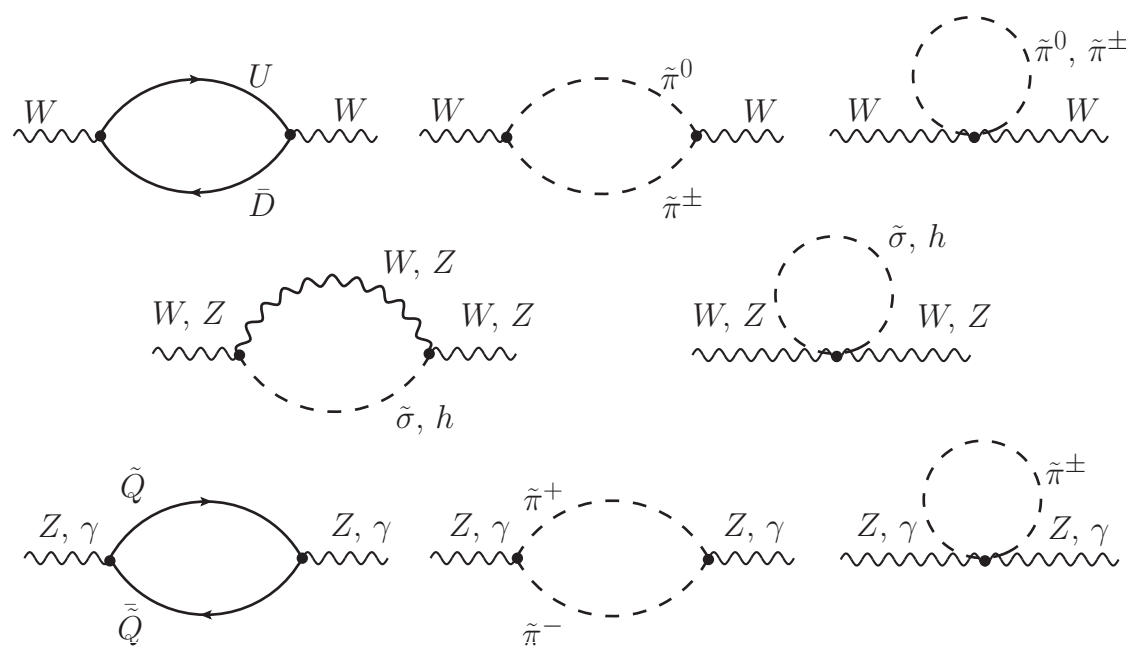

FIG. 8: The additional new (via $\tilde{\pi}, \tilde{Q}$ and $\tilde{\sigma}$ ) and modified (via Higgs boson $h$ ) contributions to the gauge bosons $Z^{0}, W^{ \pm}$and $\gamma$ vacuum polarisation functions $\delta \Pi_{\mathrm{XY}}\left(q^{2}\right)$.

Note that the modified Higgs contribution to the gauge bosons polarisation functions $\Pi_{\mathrm{XY}}^{h}\left(q^{2}, M_{h}^{2}\right)$ can be obtained by multiplying the corresponding SM result presented many times in the literature (see e.g. Ref. [48]), $\Pi_{\mathrm{XY}}^{\mathrm{SM}, \mathrm{h}}\left(q^{2}, M_{h}^{2}\right)$, by a factor of $c_{\theta}^{2}$. Also, the extra contribution due to $\tilde{\sigma}$ meson, $\Pi_{\mathrm{XY}}^{\tilde{\sigma}}\left(q^{2}, M_{\tilde{\sigma}}^{2}\right)$, can be easily obtained from the Higgs boson one, $\Pi_{\mathrm{XY}}^{h}\left(q^{2}, M_{h}^{2}\right)$, by a replacement $c_{\theta} \rightarrow s_{\theta}$ and $M_{h} \rightarrow M_{\tilde{\sigma}}$ in corresponding polarisation functions (cf. Eqs. (2.28) and (2.29) $)$. Therefore, the total contribution of the scalar states 
$\delta \Pi_{\mathrm{XY}}^{\mathrm{sc}}\left(q^{2}\right)$ to the total $\delta \Pi_{\mathrm{XY}}\left(q^{2}\right)$ defined in Eq. (3.2) reads

$$
\begin{aligned}
\delta \Pi_{\mathrm{XY}}\left(q^{2}\right) & =\delta \Pi_{\mathrm{XY}}^{\mathrm{sc}}\left(q^{2}\right)+\Pi_{\mathrm{XY}}^{\tilde{\pi}}\left(q^{2}, m_{\tilde{\pi}}^{2}\right)+\Pi_{\mathrm{XY}}^{\tilde{Q}}\left(q^{2}, M_{\tilde{Q}}^{2}\right), \\
\delta \Pi_{\mathrm{XY}}^{s c}\left(q^{2}\right) & =\Pi_{\mathrm{XY}}^{\tilde{\sigma}}\left(q^{2}, M_{\tilde{\sigma}}^{2}\right)+\Pi_{\mathrm{XY}}^{h}\left(q^{2}, M_{h}^{2}\right)-\Pi_{\mathrm{XY}}^{S M, h}\left(q^{2}, M_{h}^{2}\right) \\
& =s_{\theta}^{2} \Pi_{\mathrm{XY}}^{\mathrm{SM}, \mathrm{h}}\left(q^{2}, M_{\tilde{\sigma}}^{2}\right)-s_{\theta}^{2} \Pi_{\mathrm{XY}}^{\mathrm{SM}, \mathrm{h}}\left(q^{2}, M_{h}^{2}\right) .
\end{aligned}
$$

Apparently, $\delta \Pi_{\mathrm{XY}}^{s c}\left(q^{2}\right) \rightarrow 0$ and hence the corresponding contributions to the oblique corrections (3.3) and (3.6) turn to zero in the limit of degenerated $\tilde{\sigma}$ and $h$ masses, $M_{\tilde{\sigma}} \rightarrow M_{h}$. Also, the function $\delta \Pi_{\mathrm{XY}}^{s c}\left(q^{2}\right)$ vanish in the "no $\tilde{\sigma} h$-mixing" limit, when $s_{\theta} \rightarrow 0$, so the corresponding oblique corrections can be very small and fit the EW precision data without a significant tension.

Finally, consider the new contributions coming from $\tilde{\pi}$ and $\tilde{Q}$ loops. For illustration, below we show analytical results for the limiting "no $h \tilde{\sigma}$-mixing" scenario and degenerated technifermions implying that their constituent masses are equal $M_{U}=M_{D} \equiv M_{\tilde{Q}}$, while forthcoming numerical results and figures will be presented also for the general case with $M_{U} \neq M_{D}$ and arbitrary mixing angle. Note that if one employs an analogy with hadron physics, where the non-perturbative QCD contribution to the constituent masses of up and down quarks is much larger than their current masses, the approximate degeneracy $M_{U} \simeq M_{D}$ (or, more precisely, $\left.\Delta M_{\tilde{Q}} \equiv M_{D}-M_{U} \ll M_{U}, M_{D}\right)$ is physically reasonable and justified.

Then, the technipion and technifermion contributions can be represented in the following generic form:

$$
\Pi_{\mathrm{XY}}^{\tilde{\pi}}\left(q^{2}, m_{\tilde{\pi}}^{2}\right)=\frac{g^{2}}{24 \pi^{2}} K_{\mathrm{XY}} F_{\tilde{\pi}}\left(q^{2}, m_{\tilde{\pi}}^{2}\right), \quad \Pi_{\mathrm{XY}}^{\tilde{Q}}\left(q^{2}, M_{\tilde{Q}}^{2}\right)=\frac{g^{2} N_{c}}{24 \pi^{2}} K_{\mathrm{XY}} \kappa_{\mathrm{XY}} F_{\tilde{Q}}\left(q^{2}, M_{\tilde{Q}}^{2}\right)
$$

where $N_{\mathrm{TC}}=3$ is the number of technicolors, coefficients $K_{\mathrm{XY}}$ and $\kappa_{\mathrm{XY}}$ are shown for two different cases with $Y_{\tilde{Q}}=0$ and $Y_{\tilde{Q}}=1 / 3$ in Table I, and momentum-dependent parts are defined as

$$
\begin{aligned}
& F_{\tilde{\pi}}\left(q^{2}, m_{\tilde{\pi}}^{2}\right)=\frac{1}{3} q^{2}-2 m_{\tilde{\pi}}^{2}+2 A_{0}\left(m_{\tilde{\pi}}^{2}\right)+\frac{1}{2}\left(q^{2}-4 m_{\tilde{\pi}}^{2}\right) B_{0}\left(q^{2}, m_{\tilde{\pi}}^{2}, m_{\tilde{\pi}}^{2}\right), \\
& F_{\tilde{Q}}\left(q^{2}, M_{\tilde{Q}}^{2}\right)=-\frac{1}{3} q^{2}+2 M_{\tilde{Q}}^{2}-2 A_{0}\left(M_{\tilde{Q}}^{2}\right)+\left(q^{2}+2 M_{\tilde{Q}}^{2}\right) B_{0}\left(q^{2}, M_{\tilde{Q}}^{2}, M_{\tilde{Q}}^{2}\right),
\end{aligned}
$$

where $A_{0}\left(m^{2}\right)$ and $B_{0}\left(q^{2}, m^{2}, m^{2}\right)$ are the standard one- and two-point functions [49], respectively. Furthermore, one evaluates these functions and their derivatives for a given set of arguments and substitutes them into Eq. (3.11) and then to Eq. (3.9). Using the relations

TABLE I: Summary of coefficients $K_{\mathrm{XY}}$ and $\kappa_{\mathrm{XY}}$ in gauge bosons self-energies $\mathrm{X}, \mathrm{Y}=Z^{0}, W^{ \pm}, \gamma$ coming from $\tilde{\pi}$ and $\tilde{Q}$ loops (3.11). Two different cases for technifermion hypercharges are considered.

\begin{tabular}{|c|c|c|c|c|}
\hline$K, \kappa$ & $W W$ & $Z Z$ & $\gamma \gamma$ & $Z_{\gamma}$ \\
\hline \hline$K_{\mathrm{XY}}$ & 1 & $c_{W}^{2}$ & $s_{W}^{2}$ & $c_{W} s_{W}$ \\
\hline$\kappa_{\mathrm{XY}}, Y_{\tilde{Q}}=0$ & 1 & 1 & 1 & 1 \\
\hline$\kappa_{\mathrm{XY}}, Y_{\tilde{Q}}=1 / 3$ & 1 & $1+s_{W}^{4} / 9 c_{W}^{4}$ & $10 / 9$ & $1-s_{W}^{2} / 9 c_{W}^{2}$ \\
\hline
\end{tabular}



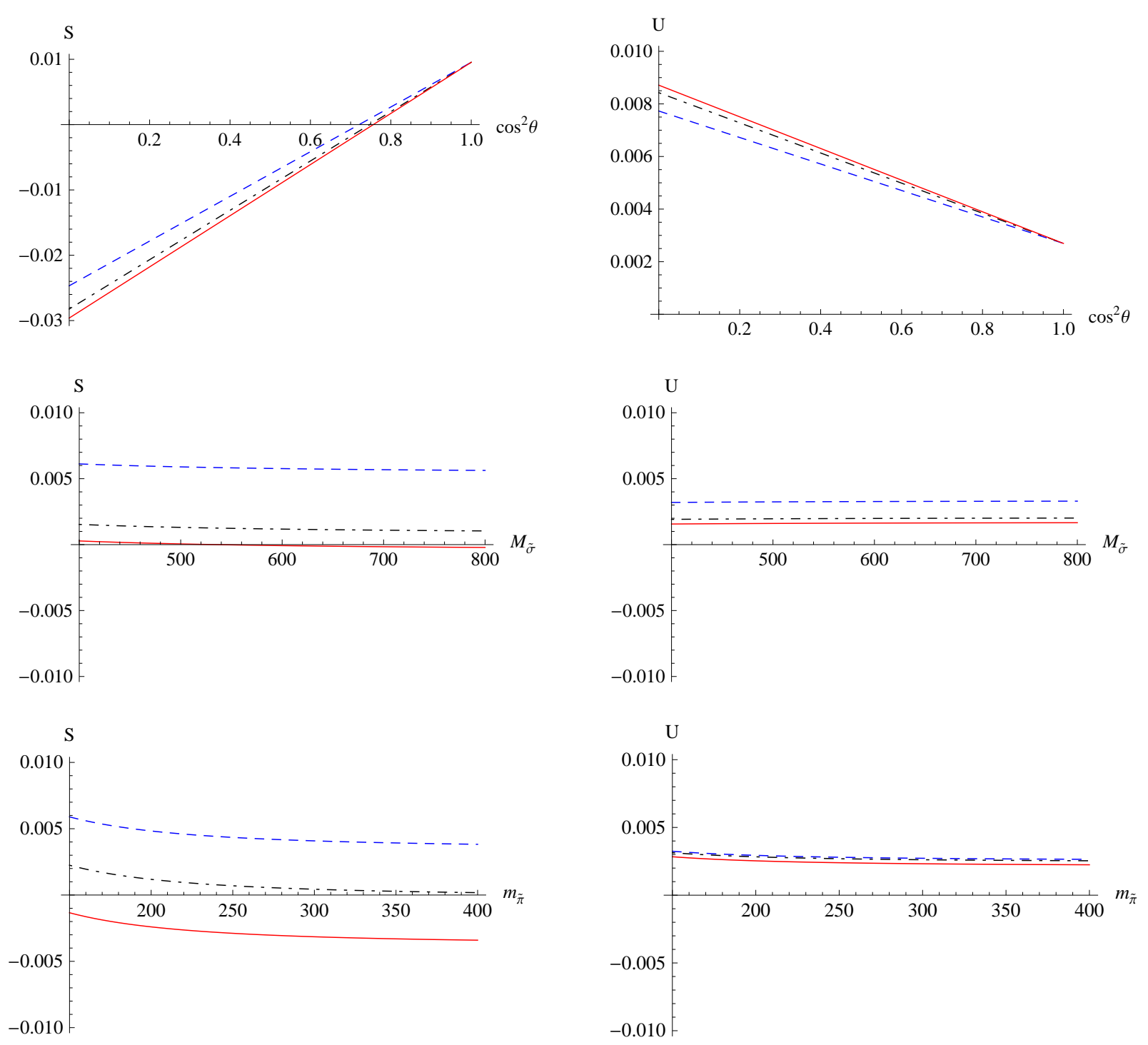

FIG. 9: The complete $S$ and $U$ parameters in the CSTC scenario (the non-minimal case with $\mu_{S}$ and $\mu_{H}$ included) as functions of (1) $\cos ^{2} \theta$ for fixed $m_{\tilde{\pi}}=150 \mathrm{GeV}, M_{\tilde{Q}} \equiv M_{U}=M_{D}=300 \mathrm{GeV}$, and $M_{\tilde{\sigma}}=400,600,800 \mathrm{GeV}$, corresponding to dashed, dash-dotted and solid lines, respectively (first row); (2) $M_{\tilde{\sigma}}$ for fixed $m_{\tilde{\pi}}=150 \mathrm{GeV}, \cos ^{2} \theta=0.9$, and $M_{\tilde{Q}}=300,500,700 \mathrm{GeV}$, corresponding to dashed, dash-dotted and solid lines, respectively (second row); and (3) $m_{\tilde{\pi}}$ for fixed $M_{\tilde{\sigma}}=500$ $\mathrm{GeV}, \cos ^{2} \theta=0.9, M_{\tilde{Q}}=300 \mathrm{GeV}$ and $\Delta M_{\tilde{Q}} \equiv M_{D}-M_{U}=0,5,10 \mathrm{GeV}$, corresponding to dashed, dash-dotted and solid lines, respectively (third row). Also, here and for other PT parameters below the sine of the mixing angle due to symmetry is chosen to be positive, $s_{\theta}>0$.

$$
B_{0}\left(0, m^{2}, m^{2}\right)=\frac{A_{0}\left(m^{2}\right)}{m^{2}}-1, \quad A_{0}\left(m^{2}\right)=m^{2}\left(\frac{1}{\bar{\varepsilon}}+1-\ln \frac{m^{2}}{\mu^{2}}\right),
$$

it can be checked directly that $F_{\tilde{\pi}}\left(0, m_{\tilde{\pi}}^{2}\right)=0$ and $F_{\tilde{Q}}\left(0, M_{\tilde{Q}}^{2}\right)=0$ which means that technipions and degenerated technifermions do not contribute to the $T$-parameter, i.e. $T^{\tilde{\pi}}=T^{\tilde{Q}}=0$ automatically. The only contribution to the $T$-parameter comes from the scalar sector of the theory: $\tilde{\sigma}$ loops and modified Higgs loops given by Eq. (3.10).

The $S$ and $U$ parameters calculation becomes especially transparent if one works in the 
linear order in $q^{2}$ power expansion and applies an approximate relation (3.5). For this purpose, let us consider the simplest case of degenerated technifermion sector with $Y_{\tilde{Q}}=0$. Then, having $\Pi_{\mathrm{XY}}^{\tilde{\pi}}\left(0, m_{\tilde{\pi}}^{2}\right)=0$ and $\Pi_{\mathrm{XY}}^{\tilde{Q}}\left(0, m_{\tilde{Q}}^{2}\right)=0$ for any $\mathrm{X}, \mathrm{Y}$ we observe that the $\tilde{\pi}$ and $\tilde{Q}$ contributions to $S$ and $U$ parameters also vanish for $Y_{\tilde{Q}}=0$ in the linear order in $q^{2}$. Indeed, using the corresponding $K_{\mathrm{XY}}$ and $\kappa_{\mathrm{XY}}$ coefficients from Table I, we explicitly see that

$$
\begin{aligned}
& \frac{\alpha S^{\tilde{\pi}+\tilde{Q}}}{4 s_{W}^{2} c_{W}^{2}}=f\left(M_{Z}^{2}, m_{\tilde{\pi}}^{2}, M_{\tilde{Q}}^{2}\right) \cdot\left[c_{W}^{2}-\frac{c_{W}^{2}-s_{W}^{2}}{c_{W} s_{W}} \cdot c_{W} s_{W}-s_{W}^{2}\right]=0, \\
& \frac{\alpha U^{\tilde{\pi}+\tilde{Q}}}{4 s_{W}^{2}}=f\left(M_{Z}^{2}, m_{\tilde{\pi}}^{2}, M_{\tilde{Q}}^{2}\right) \cdot\left[1-c_{W}^{4}-s_{W}^{4}-2 c_{W}^{2} s_{W}^{2}\right]=0,
\end{aligned}
$$

where $f\left(m_{1}^{2}, m_{2}^{2}, m_{3}^{2}\right)$ is some finite regular function of the respective mass scales. We sum-
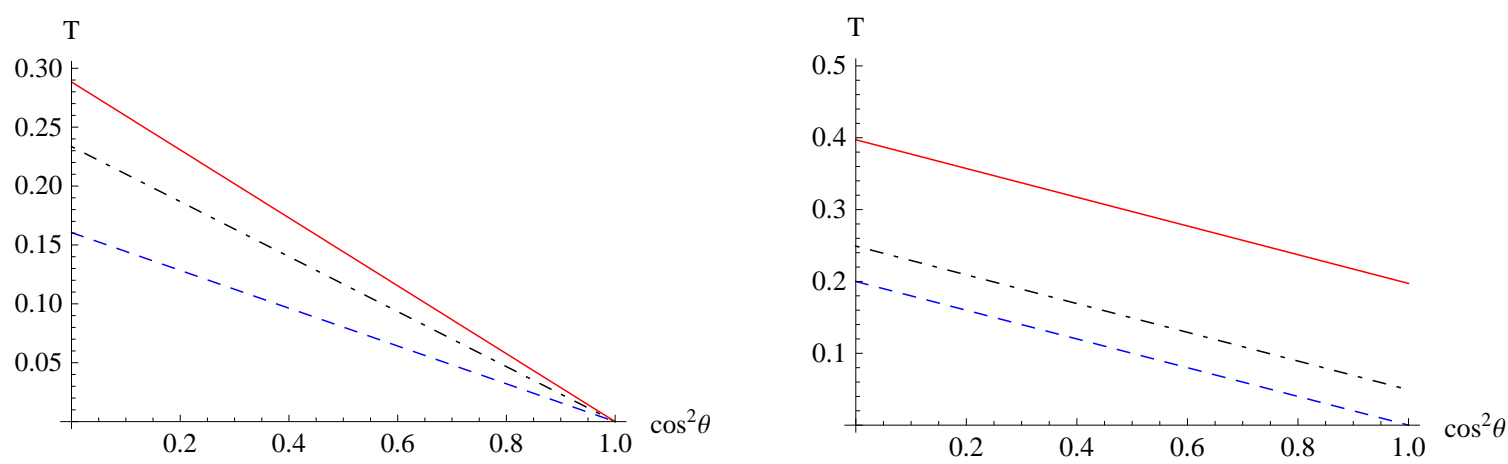

FIG. 10: The complete $T$ parameter in the CSTC scenario (the non-minimal case with $\mu_{S}$ and $\mu_{H}$ included) as function of $\cos ^{2} \theta$ for two different cases: (1) $\Delta M_{\tilde{Q}}=0$ and $M_{\tilde{\sigma}}=400,600,800$ $\mathrm{GeV}$, corresponding to dashed, dash-dotted and solid lines, respectively (left panel); (2) $M_{\tilde{\sigma}}=500$ $\mathrm{GeV}$, and $\Delta M_{\tilde{Q}} \equiv M_{D}-M_{U}=0,5,10 \mathrm{GeV}$, corresponding to dashed, dash-dotted and solid lines, respectively (right panel). The $T$ parameter does not depend on degenerated $M_{\tilde{Q}} \equiv M_{U}=M_{D}$ mass and $m_{\tilde{\pi}}$.

marize that the only contribution to the $S, T, U$ parameters (in the simplest scenario with $Y_{\tilde{Q}}=0$ and in the linear order in $q^{2}$ ) comes from scalar loops given by Eq. (3.10). This result is different from traditional TC-based scenarios with chiral-nonsymmetric weak interactions, where $S$-parameters does not vanish and is equal to a relatively large constant, even in the limit of infinitely heavy technifermions [15]. In the considering CSTC scenario this problem does not appear at all.

The calculations in more elaborated case with the SM-like technifermion hypercharge $Y_{\tilde{Q}}=1 / 3$ are less transparent and more cumbersome. Remarkably enough, in this case $S^{\tilde{\pi}+\tilde{Q}}$ and $U^{\tilde{\pi}+\tilde{Q}}$ are not zeroth any longer, but still strongly suppressed. Since in the first, linear, order in $q^{2}$ power expansion technipions and technifermions do not contribute or contribute very little, it is worth to go beyond this approximation, also incorporating $V, W, X$ parameters into the analysis. Keeping the degeneracy condition $\Delta M_{\tilde{Q}}=0$, we 
have $T^{\tilde{\pi}+\tilde{Q}}=0$, as shown above, and other parameters read

$$
\begin{aligned}
S^{\tilde{\pi}+\tilde{Q}} & =\frac{2 c_{W}^{4}}{3 \pi}\left\{\frac{1}{3}-\beta_{Z}^{\tilde{\pi}}\left(1-\phi_{Z}^{\tilde{\pi}}\right)+N_{\mathrm{TC}}\left(1+\frac{s_{W}^{4}}{9 c_{W}^{4}}\right)\left[-\frac{1}{3}+\left(3+\beta_{Z}^{\tilde{Q}}\right)\left(1-\phi_{Z}^{\tilde{Q}}\right)\right]\right\} \\
U^{\tilde{\pi}+\tilde{Q}} & =\frac{2}{3 \pi}\left\{\frac{1}{3}\left(1-c_{W}^{4}\right)-\beta_{W}^{\tilde{\pi}}\left(1-\phi_{W}^{\tilde{\pi}}\right)+c_{W}^{4} \beta_{Z}^{\tilde{\pi}}\left(1-\phi_{Z}^{\tilde{\pi}}\right)\right. \\
& \left.+N_{\mathrm{TC}}\left[-\frac{1}{3}\left(1-c_{W}^{4}-\frac{1}{9} s_{W}^{4}\right)+\left(3+\beta_{W}^{\tilde{Q}}\right)\left(1-\phi_{W}^{\tilde{Q}}\right)-\left(c_{W}^{4}+\frac{1}{9} s_{W}^{4}\right)\left(3+\beta_{Z}^{\tilde{Q}}\right)\left(1-\phi_{Z}^{\tilde{Q}}\right)\right]\right\} \\
V^{\tilde{\pi}+\tilde{Q}} & =\frac{c_{W}^{2}}{6 \pi s_{W}^{2}}\left\{-\frac{1}{2} M_{Z}^{2} \beta_{Z}^{\tilde{\pi}} B_{0}^{\prime}\left(M_{Z}^{2}, m_{\tilde{\pi}}^{2}, m_{\tilde{\pi}}^{2}\right)+\left(\beta_{Z}^{\tilde{\pi}}+1\right)\left(1-\phi_{Z}^{\tilde{\pi}}\right)\right. \\
& \left.+N_{\mathrm{TC}}\left(1+\frac{s_{W}^{4}}{9 c_{W}^{4}}\right)\left[\frac{1}{2} M_{Z}^{2}\left(3+\beta_{Z}^{\tilde{Q}}\right) B_{0}^{\prime}\left(M_{Z}^{2}, M_{\tilde{Q}}^{2}, M_{\tilde{Q}}^{2}\right)-\left(1+\beta_{Z}^{\tilde{Q}}\right)\left(1-\phi_{Z}^{\tilde{Q}}\right)\right]\right\} \\
W^{\tilde{\pi}+\tilde{Q}} & =\frac{1}{6 \pi s_{W}^{2}}\left\{-\frac{1}{2} M_{W}^{2} \beta_{W}^{\tilde{\pi}} B_{0}^{\prime}\left(M_{W}^{2}, m_{\tilde{\pi}}^{2}, m_{\tilde{\pi}}^{2}\right)+\left(\beta_{W}^{\tilde{\pi}}+1\right)\left(1-\phi_{W}^{\tilde{\pi}}\right)\right. \\
& \left.+N_{\mathrm{TC}}\left[\frac{1}{2} M_{W}^{2}\left(3+\beta_{W}^{\tilde{Q}}\right) B_{0}^{\prime}\left(M_{W}^{2}, M_{\tilde{Q}}^{2}, M_{\tilde{Q}}^{2}\right)-\left(1+\beta_{W}^{\tilde{Q}}\right)\left(1-\phi_{W}^{\tilde{Q}}\right)\right]\right\} \\
X^{\tilde{\pi}+\tilde{Q}} & =\frac{c_{W}^{2}}{6 \pi}\left\{-\frac{1}{3}+\beta_{Z}^{\tilde{\pi}}\left(1-\phi_{Z}^{\tilde{\pi}}\right)+N_{\mathrm{TC}}\left(1-\frac{s_{W}^{2}}{9 c_{W}^{2}}\right)\left[\frac{1}{3}-\left(3+\beta_{Z}^{\tilde{Q}}\right)\left(1-\phi_{Z}^{\tilde{Q}}\right)\right]\right\}
\end{aligned}
$$

where

$$
\begin{aligned}
& \beta_{Z, W}^{\tilde{\pi}}=\frac{4 m_{\tilde{\pi}}^{2}}{M_{Z, W}^{2}}-1>0, \quad \beta_{Z, W}^{\tilde{Q}}=\frac{4 M_{\tilde{Q}}^{2}}{M_{Z, W}^{2}}-1>0, \\
& \phi_{Z, W}^{\tilde{\pi}, \tilde{Q}}=\left(\beta_{Z, W}^{\tilde{\pi}, \tilde{Q}}\right)^{1 / 2} \arctan \left(\beta_{Z, W}^{\tilde{\pi}, \tilde{Q}}\right)^{-1 / 2}, \quad B_{0}^{\prime}\left(M^{2}, m^{2}, m^{2}\right)=\int_{0}^{1} d x \frac{x(1-x)}{m^{2}-M^{2} x(1-x)} .
\end{aligned}
$$

In order to constrain the viability of the CSTC model, let us look at the complete EW precision $\mathrm{PT}(S, T, U, V, W, X)$ parameters in general case, appearing due to both the modifications in the scalar sector and the new states propagating in loops, as well as at their dependence on the physical parameters of the model. These are demonstrated in Figs. 9, 10, 11 and 12. In particular, we see that the $S$-parameter is always restricted by $|S|<0.03$, and can even turn to zero for small mixing angles $\sin ^{2} \theta \sim 0.2$, moderate values of $\Delta M_{\tilde{Q}} \sim 5$ $\mathrm{GeV}$ and large values of $M_{\tilde{Q}} \gtrsim 600 \mathrm{GeV}$, and this is weakly dependent on $M_{\tilde{\sigma}}$ (see Fig. 9). So, we conclude that in the CSTC there is not such a big issue to satisfy the constraints on the $S$ parameter (3.1): the predictions fit well with $\left|S^{\text {data }}\right| \lesssim 0.1$ for the whole physically reasonable parameter space.

Does this fortunate conclusion persist also for other PT parameters? The $U$ parameter is strongly suppressed too, and never exceeds 0.01 , while being rather weakly dependent on all the physical parameters except for the mixing angle, however, it never turns into zero exactly $U \gtrsim 0.002$ (see Fig. 91). Thus, both $S$ and $U$ parameters cannot be used for an efficient constraining the model parameter space at the current level of data uncertainties (3.1). The same holds true for associated oblique corrections beyond the linear $q^{2}$ power expansions, given in terms of $V, W, X$ parameters (3.6). In particular, $V$ and $W$ parameters remain of the same order of magnitude as the $S$ and $U$ parameters. They belong to the interval $0.002 \lesssim V, W \lesssim 0.01$ and are weakly dependent on physical parameters (see Fig. 11), 

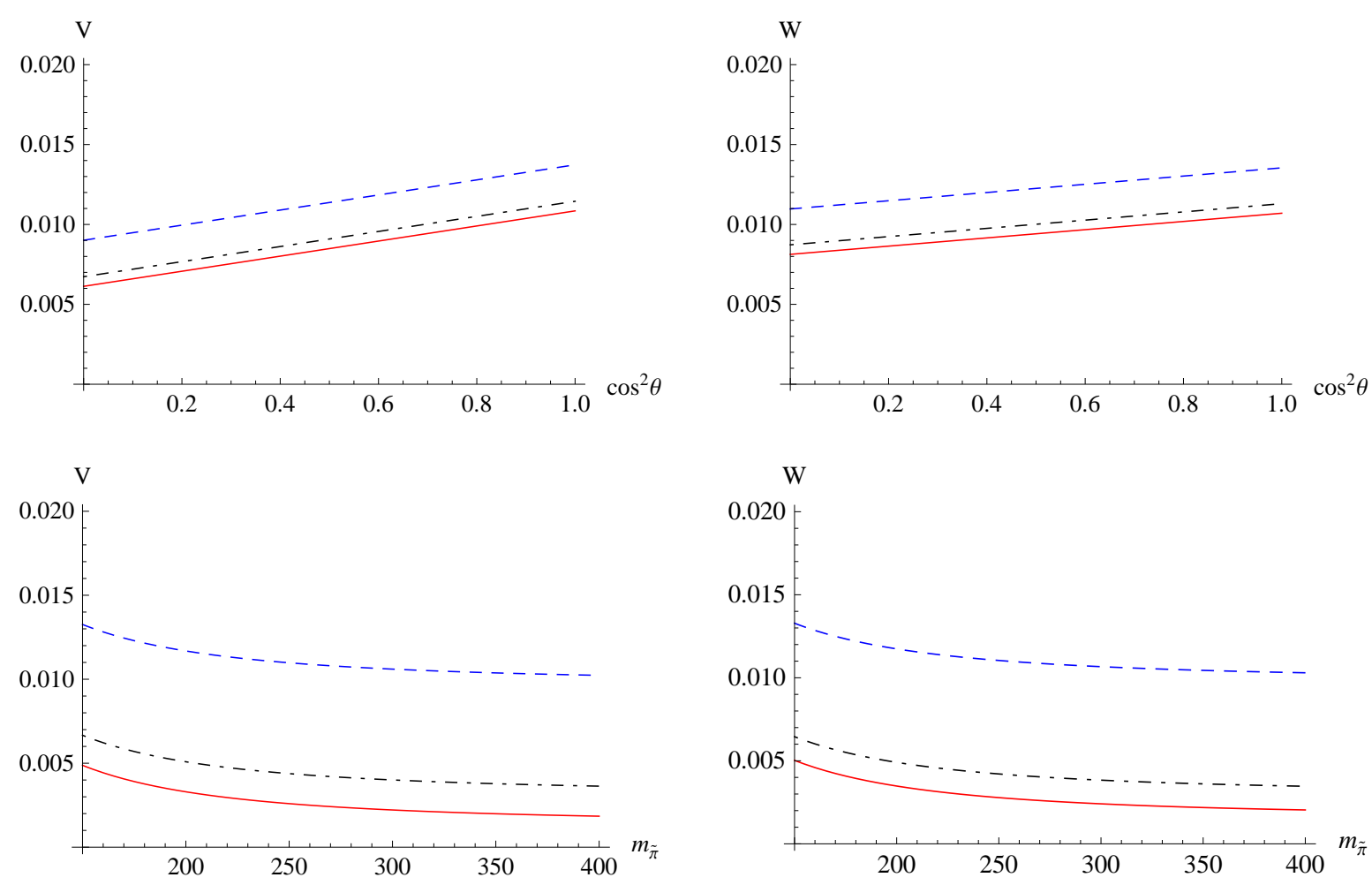

FIG. 11: The complete $V$ and $W$ parameters in the CSTC scenario (the non-minimal case with $\mu_{S}$ and $\mu_{H}$ included) as functions of (1) $\cos ^{2} \theta$ for fixed $M_{U}=M_{D}=300 \mathrm{GeV}$, and $m_{\tilde{\pi}}=150,250,350$ $\mathrm{GeV}$, corresponding to dashed, dash-dotted and solid lines, respectively (first row); and (2) $m_{\tilde{\pi}}$ for fixed $\cos ^{2} \theta=0.9$, and $M_{\tilde{Q}}=300,500,700 \mathrm{GeV}$, corresponding to dashed, dash-dotted and solid lines, respectively (second row). Both $V$ and $W$ parameters do not depend on $M_{\tilde{\sigma}}$ and $\Delta M_{\tilde{Q}}$.

whereas the $X$ parameter is even stronger suppressed, $|X| \sim 0.001$ (see Fig. 12). In general, this situation is not noticeably affected by having more than one generation of technifermions or other $N_{\mathrm{TC}}$ different from three.

The strongest bounds to the CSTC parameter space actually come from the $T$ parameter (see Fig. 10). The EW precision constraints to the $T$ parameter encoding the vector isospin breaking effects (3.1) are satisfied only for a relatively small $h \tilde{\sigma}$ mixing $\sin ^{2} \theta \lesssim 0.3$ and a small splitting between current technifermion masses $\Delta M_{\tilde{Q}} \lesssim 5 \mathrm{GeV}$. The latter is natural since similarly the relatively small splitting between the current up and down quark masses compared to their constituent masses applies also for usual QCD. In the degenerated case with $\Delta M_{\tilde{Q}}=0$ and in the "no $h \tilde{\sigma}$-mixing" limit $\cos ^{2} \theta \rightarrow 1$, the $T$-parameter vanishes identically, $T \rightarrow 0$. So, the CSTC model has enough room to fit with the EW precision data, together with tight constraints to the Higgs sector properties.

Note, the $S, U, V, W, X$ parameters are always UV finite. The $T$ parameter is finite in the degenerated case when $M_{U}=M_{D}$, whereas in general case it has logarithmic divergence proportional to the technifermion mass difference, i.e. $\operatorname{div}(T) \sim\left(M_{U}-M_{D}\right)^{2} / \varepsilon$ with a constant coefficient. Note also that the EW constraints put much stronger limits on parameter space in the case of inverse mass hierarchy in the scalar sector of the theory, i.e. assuming that technisigma is the lightest scalar particle observed at the LHC $M_{\tilde{\sigma}}<M_{h}$. In this case, the $h \tilde{\sigma}$-mixing angle has to be much closer to being vanished in order to satisfy the constraints on the corresponding oblique corrections. 


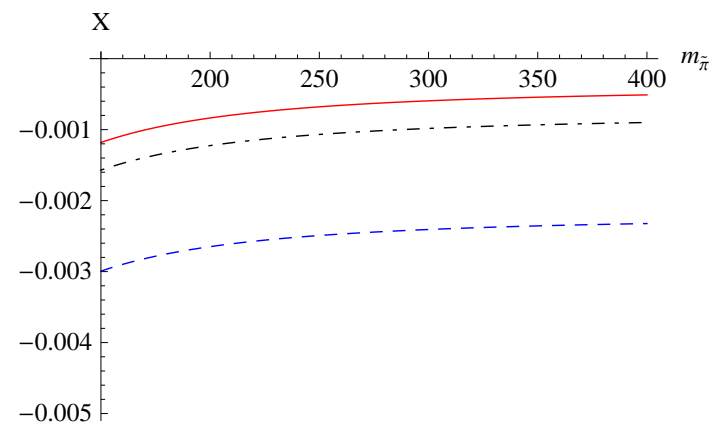

FIG. 12: The $X$ parameter $m_{\tilde{\pi}}$ for fixed $M_{\tilde{Q}}=300,500,700 \mathrm{GeV}$, corresponding to dashed, dash-dotted and solid lines, respectively. It does not depend $\cos ^{2} \theta, M_{\tilde{\sigma}}$ and $\Delta M_{\tilde{Q}}$.

\section{B. Qualitative remarks on FCNC constraints}

Another source of (less) stringent constraints onto TC models comes from the FCNCinduced processes (see e.g. Ref. [50]). In particular, here one would be interested in constraints coming from such processes as mixing in system of neutral mesons $M^{0}-\bar{M}^{0}$, as well as from rare leptonic decays of neutral mesons $M^{0} \rightarrow l \bar{l}$, etc. The semi-leptonic decays are presumably more uncertain theoretically due to larger contributions from poorly known hadronic form factors thus making it rather hard to set definite constraints to NP contributions. The flavor constraints can be very relevant for phenomenological tests of the TC models with relatively light spin-1 resonances with the same quantum numbers as the SM gauge bosons. In the considering CSTC model under discussion adopting the QCD-like mass hierarchy in the technihadron spectrum there are no light spin-1 particles; heavy vector $\tilde{\rho}$ and axial-vector $\tilde{a}_{1}$ states are considered to be decoupled from the lightest technipion and technisigma states and do not participate in processes at low momentum transfers. This is, of course, a valid approximation motivated by advances of the usual hadron physics. An extended theory which supposedly includes heavy states should then be quantitatively tested against the flavor constraints according to Ref. [50], in particular, setting up the low bounds on masses of heavy (pseudo)vector particles. However, this analysis will only be reasonable after the lightest (pseudo)scalar states have been discovered experimentally.

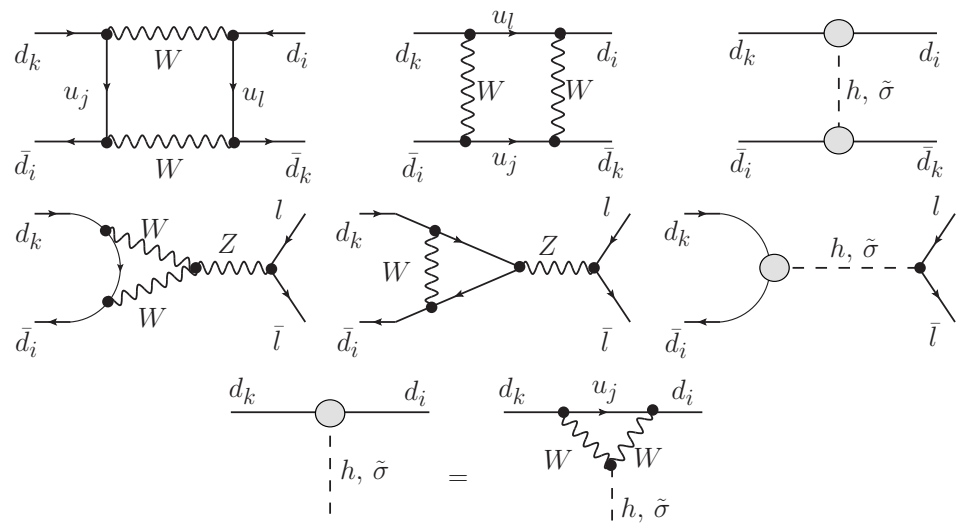

FIG. 13: Typical FCNC contributions in the CSTC model. The rightmost diagrams with scalar exchanges are the only weakly affected contributions due to a small $h \tilde{\sigma}$-mixing and additional $\tilde{\sigma}$ meson, which are however negligibly small (see main text). 
In Fig. 13 we illustrate new contributions (besides those in the gauge bosons polarisations) to typical FCNC processes (rightmost diagrams), along with the standard part (first two diagrams on the left hand side). These diagrams describe the short-distance contributions, which dominate the FCNC observables for heavy flavor mesons (for instance, $B_{d}^{0}, B_{s}^{0}$ ). In the framework of the CSTC model, an additional effect comes only from the $h \tilde{\sigma}$-mixing, whereas technipions and technifermions can only contribute to the gauge bosons polarisation functions inside the loop propagators.

The qualitative analysis of these contributions reveals that these contributions are strongly suppressed due to the following arguments:

- the typical contributions from two-loop FCNC effects with the Higgs boson in the $t$ channel in neutral mesons $M^{0}-\bar{M}^{0}$ mixing is extremely small, and usually neglected in the SM calculations. An additional (small) mixing with the heavy $\tilde{\sigma}$ field can not change this situation noticeably;

- in the case of rare (semi)leptonic decays of the Higgs boson, as well as $\tilde{\sigma}$ meson, Yukawa couplings to leptons are usually very small $\left(\sim g m_{l} / M_{W}\right)$, and the corresponding contributions are also neglected;

- in all cases the $\tilde{\sigma}$ contributions are additionally suppressed by a large technisigma mass compared to vector boson masses, $M_{\tilde{\sigma}} \gg M_{W, Z}$;

- an extra (double) suppression in the limit of small $h \tilde{\sigma}$-mixing by $s_{\theta}^{2} \ll 1$ factor in the amplitude;

- the higher-loop effect from the technipions and technifermions contributing only to the gauge bosons polarisation functions inside the loop propagators vanish at small loop momentum $q^{2} \rightarrow 0$, but otherwise is expected to be extremely small.

We conclude, that the most stringent constraints on the parameter space in the considering CSTC scenario come from the $T$-parameter which sets the upper bound to the $h \tilde{\sigma}$-mixing (see previous Section). Now, we turn to a discussion of the phenomenological consequences of the CSTC.

\section{COLLIDER PHENOMENOLOGY OF THE CSTC}

\section{A. Higgs boson production and decay}

The properties of the Higgs sector in the SM are the subject of intensive studies and discussions in the light of the latest data from the LHC [1, 2, 4]. The Higgs couplings are expected to be rather sensitive to NP contributions, and could therefore serve as a good probe of physics beyond the SM. However, it is important to notice here that even though the Higgs boson may look standard according to the latest observations and studies, this does not totally exclude possible role of NP in the EWSB and, in particular, in its underlined dynamical reasons. Further in this subsection we will examine basic possible signatures of the CSTC in Higgs boson observables.

Consider first the simplest $s$-channel subprocess of the Higgs boson production with subsequent decay into final states, i.e. $a b \rightarrow h \rightarrow X Y$. Typically, the initial states of this

subprocess are $a b=g g, Z Z, W W$ and the final states are $X Y=f \bar{f}, W W^{*}, Z Z^{*}, \gamma \gamma$, and 
$\gamma Z$. As is seen from the physical Lagrangian of the Higgs boson interactions (2.27) and (2.28), the standard tree-level $h V V$ and $h f \bar{f}$ couplings are modified by a common factor $c_{\theta}$ only caused by a mixing with heavy technisigma state.

For $a b=g g$, the $g g h$ and $g g \tilde{\sigma}$ couplings are loop-induced via heavy quarks, and there are no additional loop diagrams can contribute here in the framework of CSTC. Hence, in the Higgs boson production amplitude there always comes an extra factor $c_{\theta}$ compared to the corresponding SM amplitude. Further, the first three Higgs decay channels $X Y=f \bar{f}, W W^{*}$, $Z Z^{*}$ are the tree-level ones, with another factor $c_{\theta}$ in the amplitude, so the corresponding amplitudes $V V \rightarrow h \rightarrow f \bar{f}, W W^{*}, Z Z^{*}$ can only be different w.r.t. the $\mathrm{SM}$ ones by a factor of $c_{\theta}^{2}$ only (or a factor of $s_{\theta}^{2}$ in the case of intermediate $\tilde{\sigma}$ meson). But this is true only if one considers the $s$-channel production process far from the resonance, $\hat{s}^{\text {res }}=M_{h}^{2}$ (or $\hat{s}^{\text {res }}=M_{\tilde{\sigma}}^{2}$ for the intermediate $\tilde{\sigma}$ meson). However, in the resonance region the modifications of the $\mathrm{SM}$ amplitudes can be different from mere mixing factor multiplication.

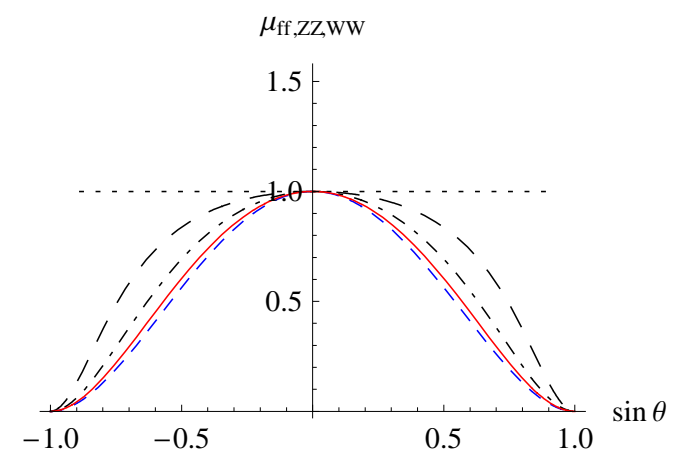

FIG. 14: The Higgs boson signal strength in the tree-level $f \bar{f}, Z Z^{*}$ and $W W^{*}$ channels

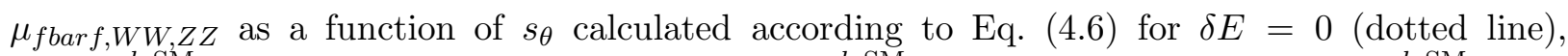
$\delta E=\Gamma_{\text {tot }}^{h, \mathrm{SM}} \simeq 4.03 \mathrm{MeV}$ (dash-dotted line), $\delta E=\Gamma_{\text {tot }}^{h, \mathrm{SM}} / 2$ (long-dashed line), $\delta E=2 \Gamma_{\text {tot }}^{h, \mathrm{SM}}$ (solid line), and $c_{\theta}^{4}=\left(1-s_{\theta}^{2}\right)^{2}$ curve is also shown for reference (dashed line).

In order to calculate the $s$-channel cross section for the scalar Higgs boson (and $\tilde{\sigma}$ meson) production with two-particle final states one starts from the universal factorized formula which reproduces the well-known narrow-width approximation formula and has been proven to be exact in the framework of the unstable particles model with smeared mass shell (see e.g. Ref. [51])

$$
\sigma(a b \rightarrow h(q), \tilde{\sigma}(q) \rightarrow X Y)=\frac{16 \pi k_{h, \tilde{\sigma}}}{k_{a} k_{b} \bar{\lambda}^{2}\left(m_{a}, m_{b} ; q\right)} \frac{\Gamma(h(q), \tilde{\sigma}(q) \rightarrow a b) \Gamma(h(q), \tilde{\sigma}(q) \rightarrow X Y)}{\left[q^{2}-M_{h, \tilde{\sigma}}^{2}\right]^{2}+\left[q \Gamma_{h, \tilde{\sigma}}^{t o t}(q)\right]^{2}}(4.1)
$$

where $q=p_{a}+p_{b}$ is the total $s$-channel 4 -momentum, $k_{a}=2 J_{a}+1$ is the number of polarisation states, and $J_{a}$ is the spin of particle $a$ (i.e. $k_{h, \tilde{\sigma}}=1$ ), and

$$
\bar{\lambda}^{2}\left(m_{a}, m_{b} ; q\right)=1-2 \frac{m_{a}^{2}+m_{b}^{2}}{q^{2}}+\frac{\left(m_{a}^{2}-m_{b}^{2}\right)^{2}}{q^{4}}
$$

is the normalized Källen function. A good estimate of modifications in $h, \tilde{\sigma}$ couplings in the resonance region where $q^{2} \simeq M_{h, \tilde{\sigma}}^{2}$ can thus be obtained from the formula

$$
\sigma(a b \rightarrow h, \tilde{\sigma} \rightarrow X Y) \simeq \frac{16 \pi}{k_{a} k_{b} \bar{\lambda}^{2}\left(m_{a}, m_{b} ; m_{h, \tilde{\sigma}}\right) M_{h, \tilde{\sigma}}^{2}} \operatorname{Br}(h, \tilde{\sigma} \rightarrow a b) \cdot \operatorname{Br}(h, \tilde{\sigma} \rightarrow X Y) .
$$




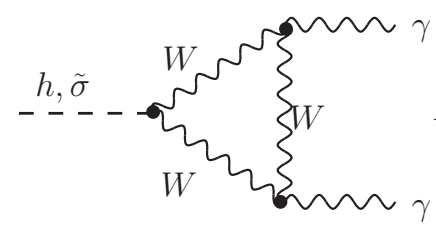

(a)

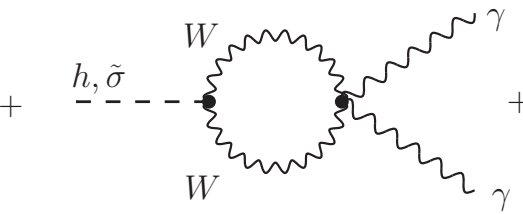

(b)

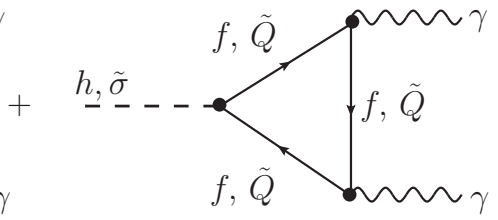

(c)

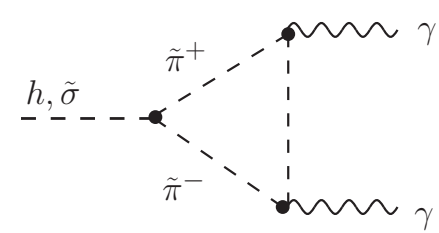

(d)

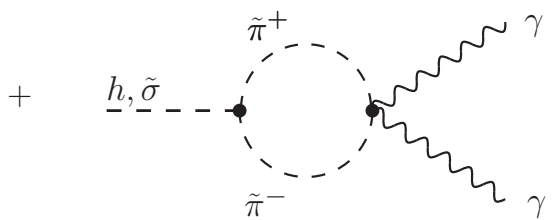

$(\mathbf{e})$

FIG. 15: Typical one-loop contributions to the $h, \tilde{\sigma} \rightarrow \gamma \gamma$ decay channel in the CSTC.

As was mentioned above, the Higgs couplings to SM fermions and vector bosons in the considering scenario contain extra $c_{\theta}$ factor compared to the SM ones, so in the resonance region we have for decay widths and branching fractions to a good accuracy

$$
\frac{\Gamma_{t o t}^{h, \text { mod }}}{\Gamma_{\text {tot }}^{h, \mathrm{SM}}} \simeq c_{\theta}^{2}, \quad \frac{\mathrm{Br}^{\text {mod }}(h \rightarrow X Y)}{\mathrm{Br}^{\mathrm{SM}}(h \rightarrow X Y)} \simeq 1, \quad X Y=f \bar{f}, W W^{*}, Z Z^{*},
$$

i.e. for all Born-level Higgs/technisigma decays which strongly dominate in the total decay width. This reveals the fact that the Higgs branching ratios, in fact, in the SM and in the considering CSTC scenario are the same. Thus, according to Eq. (4.3) the ratio between the resonant cross sections in the considering model to the SM one is close to unity

$$
\mu_{f \bar{f}, Z Z, W W}^{\mathrm{res}}=\frac{\sigma^{\bmod }\left(V V \rightarrow h(q) \rightarrow \bar{f} f, Z Z^{*}, W W^{*}\right)}{\sigma^{\mathrm{SM}}\left(V V \rightarrow h(q) \rightarrow \bar{f} f, Z Z^{*}, W W^{*}\right)} \simeq 1, \quad q^{2} \simeq M_{h}^{2} .
$$

which are essentially the Higgs boson signal strengths in respective channels which were measured earlier at the LHC and no significant deviations from the SM have been found.

In fact, experimentally one never measures events exactly at the resonance peak position $q^{2}=M_{h}^{2}$, but one rather has a smearing of the resonance by e.g. detector conditions. In this case, a more precise estimation of the Higgs boson signal strength is given by the ratio of the cross sections integrated (or averaged) over the energy resolution of an experiment $\delta E$ which can be comparable or exceeds the small Higgs boson decay width in the SM, $\delta E \geq \Gamma_{\text {tot }}^{h, \mathrm{SM}} \simeq 4.03 \mathrm{MeV}\left(\right.$ at $\left.M_{h} \simeq 125 \mathrm{GeV}\right)$ [12], i.e.

$$
\begin{aligned}
\mu_{\mathrm{XY}}(\delta E)=\frac{\int_{M_{h}-\delta E}^{M_{h}+\delta E} \sigma_{\mathrm{XY}}^{\text {mod }}(q) d q}{\int_{M_{h}-\delta E}^{M_{h}+\delta E} \sigma_{\mathrm{XY}}^{\mathrm{SM}}(q) d q} & \simeq \frac{\Gamma^{\text {mod }}(h \rightarrow a b) \Gamma^{\text {mod }}(h \rightarrow X Y)}{\Gamma^{\mathrm{SM}}(h \rightarrow a b) \Gamma^{\mathrm{SM}}(h \rightarrow X Y)} \\
& \times \frac{\int_{M_{h}-\delta E}^{M_{h}+\delta E}\left[\left(q^{2}-M_{h}^{2}\right)^{2}+q^{2}\left(\Gamma_{t o t}^{h, \mathrm{SM}}\right)^{2}\right] d q}{\int_{M_{h}-\delta E}^{M_{h}+\delta E}\left[\left(q^{2}-M_{h}^{2}\right)^{2}+q^{2}\left(\Gamma_{\text {tot }}^{h, m o d}\right)^{2}\right] d q},
\end{aligned}
$$

whose values have to be compared to the measured ones. The last part of the formula above is fulfilled approximately and valid to a good accuracy for $\delta E \gg \Gamma_{\text {tot }}^{h, \mathrm{SM}}$ which is the case in 

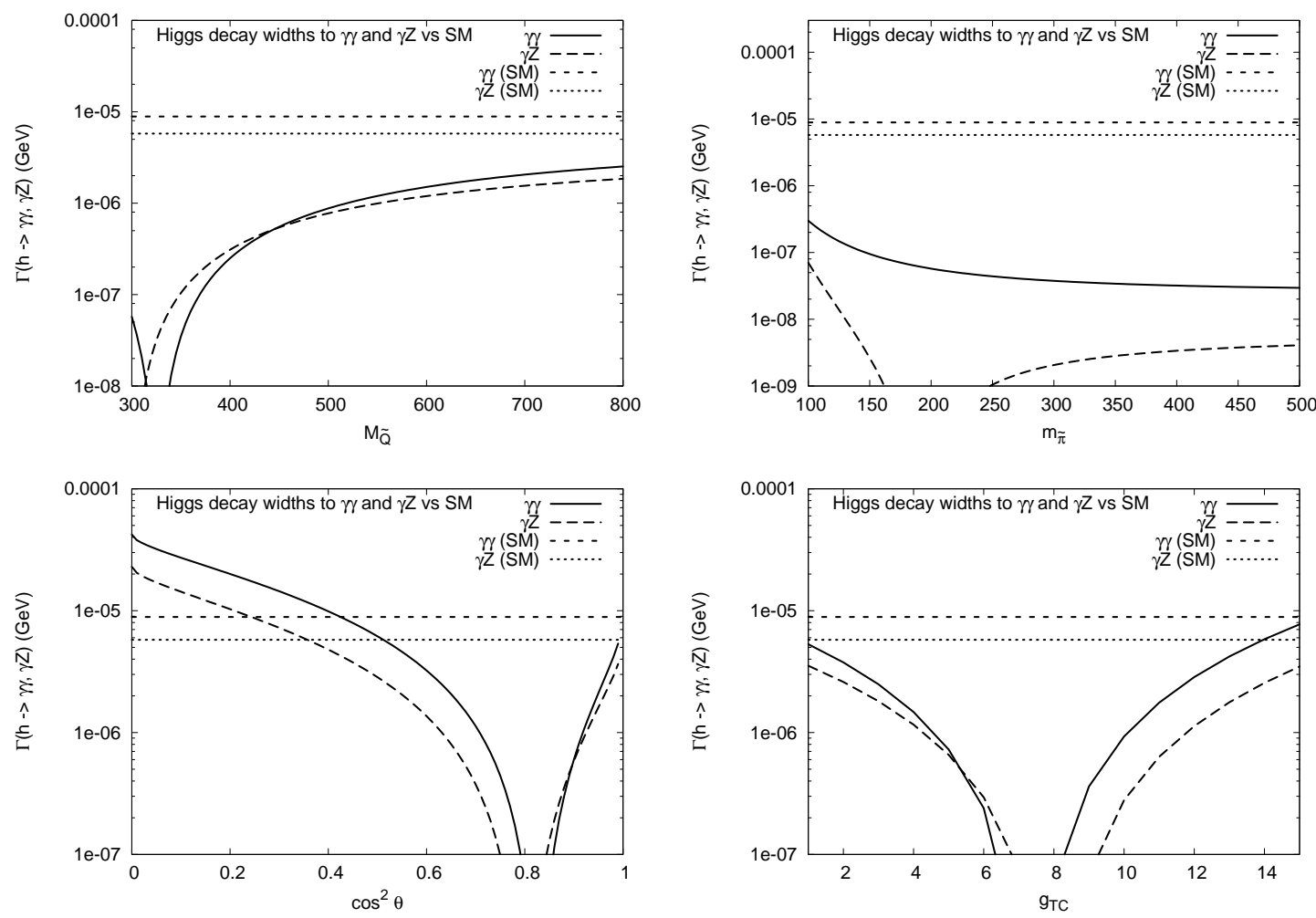

FIG. 16: The Higgs boson decay widths in the loop-induced $\gamma \gamma$ and $\gamma Z$ channels in the nonminimal CSTC (with scalar $\mu_{S, H}$-terms included) as functions of physical parameters of the model. The corresponding SM predictions are shown for comparison. The parameters in each figure are set as follows: (top-left) $m_{\tilde{\pi}}=200 \mathrm{GeV}, c_{\theta}^{2}=0.8$, and $g_{\mathrm{TC}}=8$; (top-left) $M_{\tilde{Q}}=300 \mathrm{GeV}$, $c_{\theta}^{2}=0.8$, and $g_{\mathrm{TC}}=8$; (bottom-left) $M_{\tilde{Q}}=300 \mathrm{GeV}, m_{\tilde{\pi}}=200 \mathrm{GeV}$, and $g_{\mathrm{TC}}=8$; (bottom-right) $M_{\tilde{Q}}=300 \mathrm{GeV}, m_{\tilde{\pi}}=200 \mathrm{GeV}$, and $c_{\theta}^{2}=0.8$. These results do not depend on $M_{\tilde{\sigma}}$, and the positive sign of the mixing angle, or $s_{\theta}>0$, is fixed here.

actual measurements. Clearly, the formula (4.6) turns into the Eq. (4.5) in the limit of very narrow $\delta$-shaped resonance, i.e. when $\delta E \ll \Gamma_{t o t}^{h, S M}$.

In Fig. 14 we show the dependence of the $\mu_{f \bar{f}, W W, Z Z}(\delta E)$ on the mixing $s_{\theta}$ for different values of the peak smearing $\delta E=0$ (short-dashed line), $\delta E=\Gamma_{\text {tot }}^{h, \mathrm{SM}} \simeq 4.03 \mathrm{MeV}$ (dashdotted line), $\delta E=2 \Gamma_{\text {tot }}^{h, \mathrm{SM}}$ (solid line), and $c_{\theta}^{4}=\left(1-s_{\theta}^{2}\right)^{2}$ curve is also shown for reference (dashed line). No smearing case with $\delta E=0$ corresponds precisely to the resonance formula (4.5) with the unit strength, while an increase in the peak smearing quickly approaches to the off-resonance result with $\mu_{f \bar{f}, W W, Z Z} \sim c_{\theta}^{4}$. Clearly, an influence of the peak smearing vanishes in the no mixing limit $s_{\theta} \rightarrow 0$.

The near-resonance approximation in the $s$-channel production process (4.5) is valid up to relatively small loop-induced contributions and higher order correction which may contain extra loop contributions with technipions, technisigma and technifermions. These extra contributions can be especially pronounced in the loop-induced $\gamma \gamma$ and $\gamma Z$ decay channels (see Fig. 15). Indeed,

$$
\mu_{\gamma \gamma}^{\mathrm{res}}=\frac{\sigma^{\bmod }(h \rightarrow \gamma \gamma)}{\sigma^{\mathrm{SM}}(h \rightarrow \gamma \gamma)} \simeq \frac{1}{c_{\theta}^{2}} \frac{\Gamma^{\bmod }(h \rightarrow \gamma \gamma)}{\Gamma^{\mathrm{SM}}(h \rightarrow \gamma \gamma)} \simeq \frac{1}{c_{\theta}^{2}} \frac{\left|A_{W}+A_{f}+A_{\tilde{\pi}}+A_{\tilde{Q}}\right|^{2}}{\left|A_{W}^{\mathrm{SM}}+A_{f}^{\mathrm{SM}}\right|^{2}}
$$



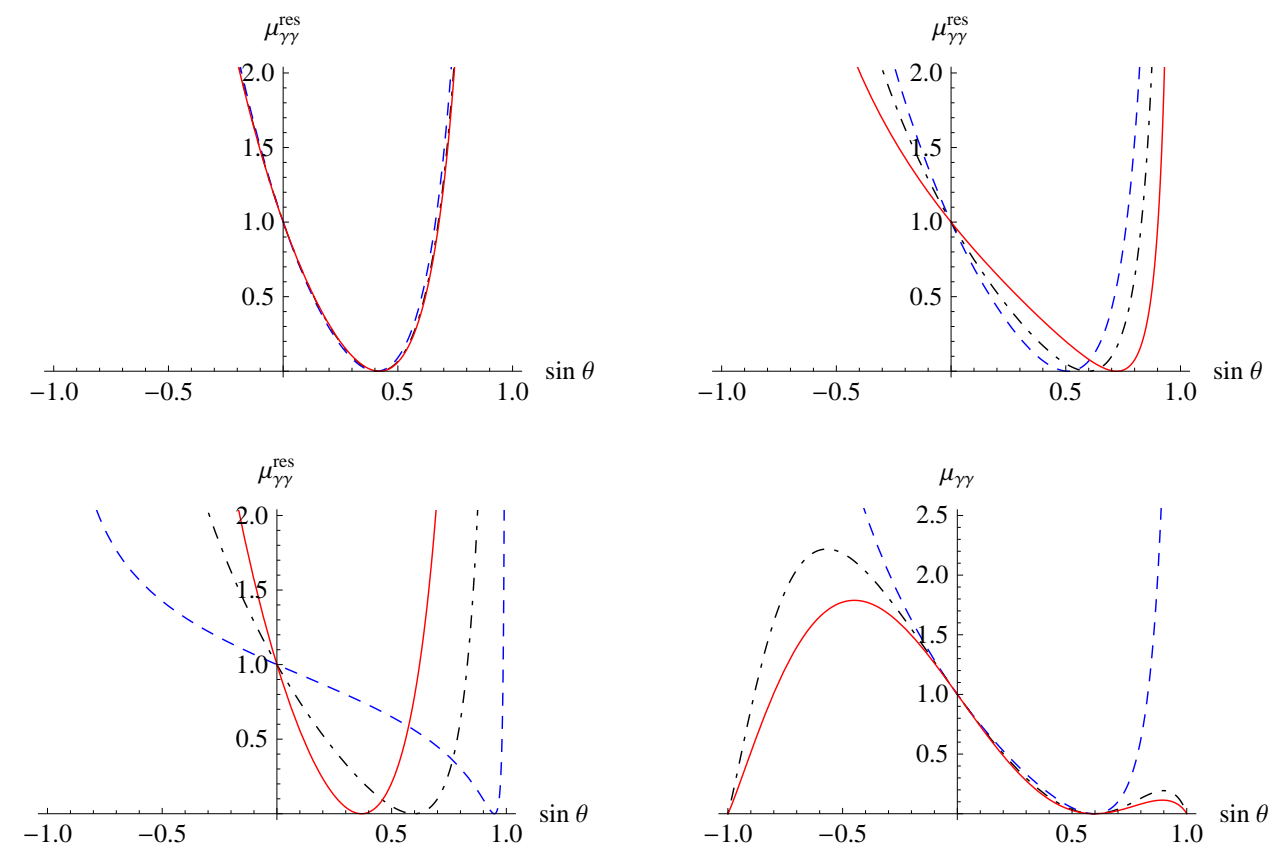

FIG. 17: Dependence of the Higgs boson signal strength in the resonance given by Eq. (4.7) in the non-minimal case of the CSTC model (with scalar $\mu_{S, H}$-terms included), $\mu_{\gamma \gamma}^{\text {res }}$, on $s_{\theta}$ for different sets of the physical parameters: (top-left) $g_{\mathrm{TC}}=8, M_{\tilde{Q}}=300 \mathrm{GeV}$, and $m_{\tilde{\pi}}=150,250,350$ $\mathrm{GeV}$, corresponding to dashed, dash-dotted and solid lines, respectively; (top-right) $g_{\mathrm{TC}}=8$, $m_{\tilde{\pi}}=150 \mathrm{GeV}$, and $M_{\tilde{Q}}=400,500,700 \mathrm{GeV}$, corresponding to dashed, dash-dotted and solid lines, respectively; (bottom-left) $m_{\tilde{\pi}}=150 \mathrm{GeV}, M_{\tilde{Q}}=500 \mathrm{GeV}$, and $g_{\mathrm{TC}}=2,8,15$, corresponding to dashed, dash-dotted and solid lines, respectively. Finally, bottom-right figure corresponds to smeared $\mu_{\gamma \gamma}(\delta E)$ given by Eq. (4.6) as a function of $s_{\theta}$ for fixed $m_{\tilde{\pi}}=150 \mathrm{GeV}, M_{\tilde{Q}}=500 \mathrm{GeV}$, $g_{\mathrm{TC}}=8$ and with different smearing parameters: no smearing $\delta E=0$ (dashed line), $\delta E=\Gamma_{\text {tot }}^{h, \mathrm{SM}} \simeq$ $4.03 \mathrm{MeV}$ (dash-dotted line), and $\delta E=1 \mathrm{GeV}$ (solid line). Here and below, $Y_{\tilde{Q}}=1 / 3$, unless noted otherwise.

where $A_{W, f, \tilde{\pi}, \tilde{Q}}$ are the amplitudes given by the SM-like $W, f$ loop diagrams (see Fig. 15 (a), (b), (c)), as well as by the new technipion $\tilde{\pi}$ and technifermion $\tilde{Q}$ loop diagrams (see Fig. 15 (c), (d), (e)). An interference effect between these contributions may be important. Notably $A_{\tilde{Q}} \sim s_{\theta}$ while $\left|A_{\tilde{\pi}}\right| \ll\left|A_{\tilde{Q}}\right|$ in general so the interference effect changes its sign depending on the sign of $s_{\theta}$ possibly giving rise to either enhancement or suppression of the $\gamma \gamma$ signal, or to the SM-like $h \rightarrow \gamma \gamma$ signal strengths in the case of a small mixing angle $s_{\theta} \ll 1$ (where the technipion loop contribution disappears as well). Since the first three diagrams, which are present in the SM, do not exist at the tree level, their sum is free of divergencies. More precisely, the divergencies are canceled between diagrams (a) and (b), and the fermion $(f$ and $\tilde{Q}$ ) loops are finite individually. We have found that the sum of technipion loops is finite as well. Also, here it is reasonable to assume that only heavy top quark loops contribute to the final result; all other fermions are strongly suppressed and thus can be neglected.

A straightforward calculation lead to the following Higgs partial decay width in the $\gamma \gamma$ channel

$$
\Gamma^{m o d}(h \rightarrow \gamma \gamma)=\frac{\alpha^{2} M_{h}}{16 \pi^{3}} \cdot\left|F_{W}+F_{t o p}+F_{\tilde{\pi}}+F_{\tilde{Q}}\right|^{2}
$$



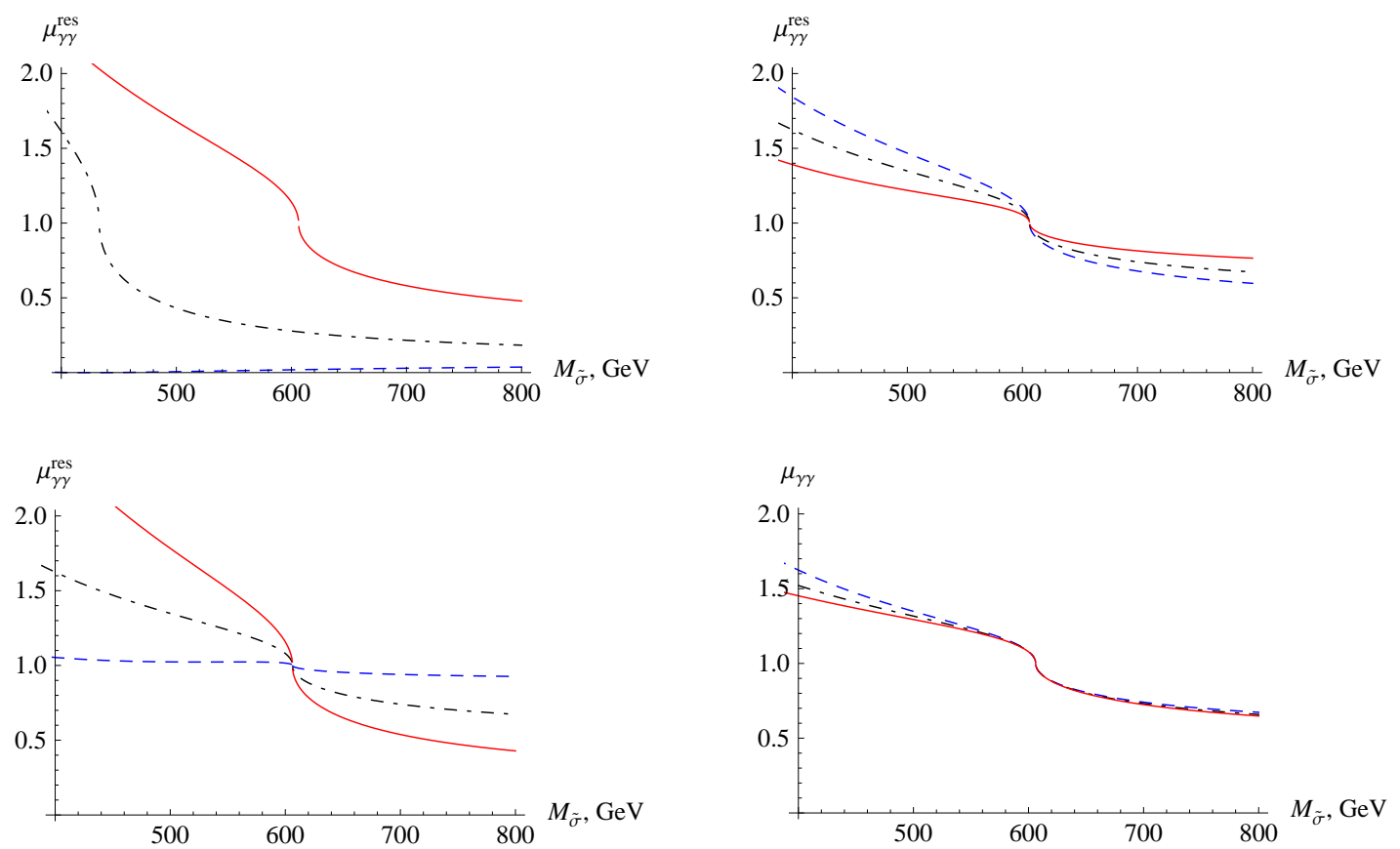

FIG. 18: Dependence of the Higgs boson signal strength in the resonance given by Eq. (4.7) in the minimal CSTC model (with scalar $\mu_{S, H}$-terms excluded), $\mu_{\gamma \gamma}^{\text {res }}$, on $M_{\tilde{\sigma}}$ for different sets of the physical parameters: (top-left) $g_{\mathrm{TC}}=8, M_{\tilde{Q}}=300 \mathrm{GeV}$, and $m_{\tilde{\pi}}=150,250,350 \mathrm{GeV}$, corresponding to dashed, dash-dotted and solid lines, respectively; (top-right) $g_{\mathrm{TC}}=8, m_{\tilde{\pi}}=$ $350 \mathrm{GeV}$, and $M_{\tilde{Q}}=400,500,700 \mathrm{GeV}$, corresponding to dashed, dash-dotted and solid lines, respectively; (bottom-left) $m_{\tilde{\pi}}=350 \mathrm{GeV}, M_{\tilde{Q}}=500 \mathrm{GeV}$, and $g_{\mathrm{TC}}=2,8,15$, corresponding to dashed, dash-dotted and solid lines, respectively. Finally, bottom-right figure corresponds to smeared $\mu_{\gamma \gamma}(\delta E)$ given by Eq. (4.6) as a function of $M_{\tilde{\sigma}}$ for fixed $m_{\tilde{\pi}}=350 \mathrm{GeV}, M_{\tilde{Q}}=500 \mathrm{GeV}$, $g_{\mathrm{TC}}=8$ and with different smearing parameters: no smearing $\delta E=0$ (dashed line), $\delta E=\Gamma_{\text {tot }}^{h, \mathrm{SM}} \simeq$ 4.03 $\mathrm{MeV}$ (dash-dotted line), and $\delta E=1 \mathrm{GeV}$ (solid line).

where $\alpha=\alpha\left(M_{Z}\right)=1 / 127.93$ is the fine structure constant adopted in all numerical calculations, and the individual contributions from $W$, top-quark, $\tilde{\pi}$ and $\tilde{Q}$ loops read, respectively,

$$
\begin{aligned}
& F_{W}=\frac{1}{8} g c_{\theta} \frac{M_{h}}{M_{W}} \cdot\left[2+3 \beta_{W}+3 \beta_{W}\left(2-\beta_{W}\right) f\left(\beta_{W}\right)\right], \\
& F_{\text {top }}=-\frac{4}{3} g c_{\theta} \frac{m_{t o p}^{2}}{M_{h} M_{W}}\left[1+\left(1-\beta_{\text {top }}\right) f\left(\beta_{\text {top }}\right)\right], \\
& F_{\tilde{\pi}}=-\frac{g_{h \tilde{\pi}}}{2 M_{h}}\left[1-\beta_{\tilde{\pi}} f\left(\beta_{\tilde{\pi}}\right)\right], \quad g_{h \tilde{\pi}}=-2\left(\lambda_{\mathrm{TC}} u s_{\theta}-\lambda v c_{\theta}\right), \\
& F_{\tilde{Q}}=-2 N_{\mathrm{TC}}\left(q_{U}^{2}+q_{D}^{2}\right) g_{\mathrm{TC}} s_{\theta} \frac{M_{\tilde{Q}}}{M_{h}}\left[1+\left(1-\beta_{\tilde{Q}}\right) f\left(\beta_{\tilde{Q}}\right)\right],
\end{aligned}
$$

where we take the number of technicolors $N_{\mathrm{TC}}=3$ in numerical calculations below, $q_{U, D}$ are the techni-up and techni-down fermion charges, and

$$
f(\beta)=\arcsin ^{2} \frac{1}{\sqrt{\beta}} \quad \beta_{X}=\frac{4 m_{X}^{2}}{M_{h}^{2}}, \quad X=W, \text { top, } \tilde{\pi}, \tilde{Q} .
$$


The non-minimal case with scalar $\mu$-terms included, the relation

$$
g_{h \tilde{\pi}}=-g_{\mathrm{TC}} s_{\theta} \frac{M_{h}^{2}-m_{\tilde{\pi}}^{2}}{M_{\tilde{Q}}}
$$

can be used (cf. Eq. (2.28)), whereas in the special case with $\mu_{S, \mathrm{H}} \rightarrow 0$ the relations (2.37) have to be employed for calculation of the $g_{h \tilde{\pi}}$ coupling. In the limit of small $h \tilde{\sigma}$-mixing, the constituent technifermion and technipion loop contributions to the Higgs boson width are suppressed by a factor of $s_{\theta}^{2} \ll 1$, so the whole expression (4.8) turns to the SM result:

$$
\Gamma^{\mathrm{SM}}(h \rightarrow \gamma \gamma)=\frac{\alpha^{2} M_{h}}{16 \pi^{3}} \cdot\left|F_{W}^{\mathrm{SM}}+F_{t o p}^{\mathrm{SM}}\right|^{2},
$$

where $F_{W, t o p}^{\mathrm{SM}}$ can be obtained from Eq. (4.9) with $c_{\theta}=1$.
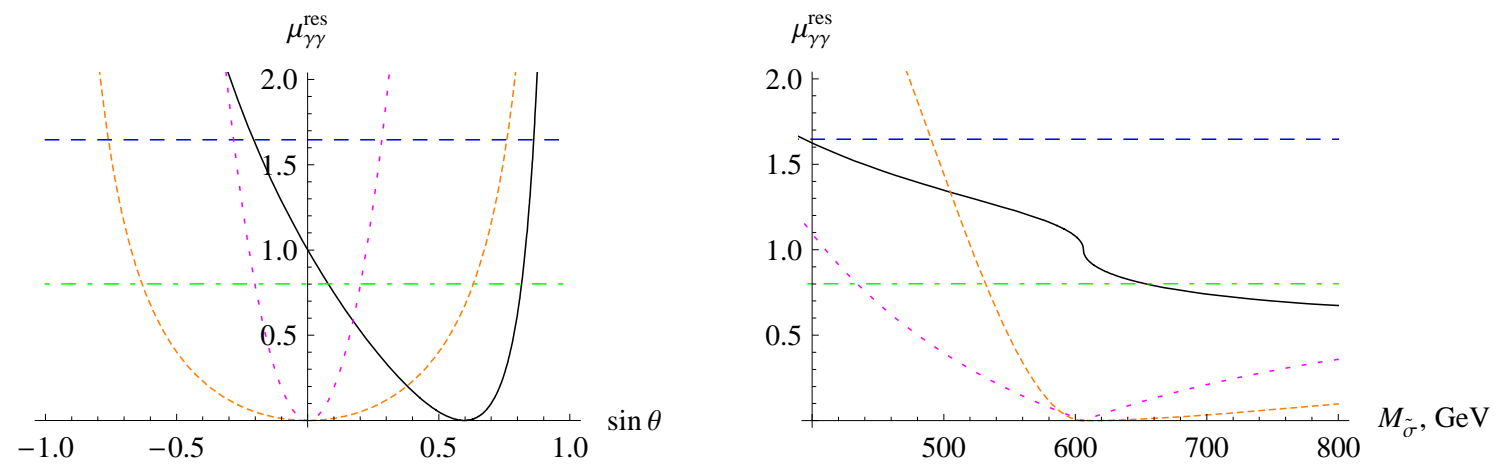

FIG. 19: Partial contributions to the $\mu_{\gamma \gamma}^{\text {res }}$ in the non-minimal CSTC (with scalar $\mu_{S, H^{-t e r m s}}$ included) as functions of $s_{\theta}$ with $m_{\tilde{\pi}}=150 \mathrm{GeV}, M_{\tilde{Q}}=500 \mathrm{GeV}$ and $g_{\mathrm{TC}}=8$ (left panel) and in the minimal CSTC without scalar $\mu_{S, H}$-terms as functions of $M_{\tilde{\sigma}}$ with $m_{\tilde{\pi}}=350 \mathrm{GeV}$, $M_{\tilde{Q}}=500 \mathrm{GeV}$ and $g_{\mathrm{TC}}=8$ (right panel), corresponding to $W$-loop (dashed lines), top quark loop $\times 10$ (dash-dotted lines), technifermion loop $\times 10$ (dotted lines), technipion loop $\times 1000$ (short-dashed lines). At the both panels, solid lines correspond to the total Higgs boson signal (resonant) strengths shown for comparison. The rescaling of the curves is made for better visibility and comparison.

The Higgs boson decay widths in the loop-induced $\gamma \gamma$ and also in the $\gamma Z$ channels in the non-minimal CSTC with scalar $\mu_{S, H}$-terms included are shown in Fig. 16 as functions of physical parameters of the model. This figure covers only $s_{\theta}>0$ region and is complimentary to Fig. 18. One notices the regions where the $\gamma \gamma$ and $\gamma Z$ widths can be very different from the SM predictions, or close to them, or even turn to zero due to a specific interference pattern. Also, the relation between $\gamma \gamma$ and $\gamma Z$ widths strongly depends on parameters. It is, however, more instructive to look directly at the Higgs signal strengths in the respective decay channels as functions of parameters, and we will primarily study the $\gamma \gamma$ channel in detail here.

In particular, let us investigate to what extent the $h \tilde{\sigma}$-mixing and the presence of the extra new $\tilde{\pi}$ and $\tilde{Q}$ states in loops affects the resonance Higgs signal strength in the $\gamma \gamma$ channel $\mu_{\gamma \gamma}^{\text {res }}$ and its smearing, given by Eqs. (4.7) and (4.6), respectively. For this purpose, in Fig. 17 we show the Higgs boson signal strength in the $\gamma \gamma$ channel in the resonance region $\mu_{\gamma \gamma}^{\text {res }}\left(s_{\theta}\right)$ given 
by Eq. (4.7) in the non-minimal case of the CSTC model with scalar $\mu_{S, H^{-}}$terms included. The $\mu_{\gamma \gamma}^{\text {res }}\left(s_{\theta}\right)$ weakly depends on $m_{\tilde{\pi}}$ value. It also turns into zero at some $s_{\theta}^{*}>0$, which increases with $M_{\tilde{Q}}$ and decreases with $g_{\mathrm{TC}}$. Note that there is no symmetry $s_{\theta} \rightarrow-s_{\theta}$. In general, for $s_{\theta}<0$, we always have in the resonance $\mu_{\gamma \gamma}^{\text {res }}\left(s_{\theta}\right)>1$, while smearing over the resonance can change this. Also, smearing does not change significantly $\mu_{\gamma \gamma}^{\text {res }}\left(s_{\theta}\right)$ at small smearing angles $s_{\theta} \rightarrow 0$. The signal strength is close to unity for two different cases of the mixing angle: in the no $h \tilde{\sigma}$-mixing limit $s_{\theta} \rightarrow 0$ and for $s_{\theta} \sim 0.5-0.7$, while the latter is much more fine-tuned do to a sharp behavior of $\mu_{\gamma \gamma}^{\text {res }}\left(s_{\theta}\right)$; the third configuration at negative $s_{\theta}$ appears due to a resonance smearing described above. Note, that any relatively large mixing configurations with $s_{\theta}^{2}>0.4$ are excluded by EW precision constraints on $T$-parameter (see above).

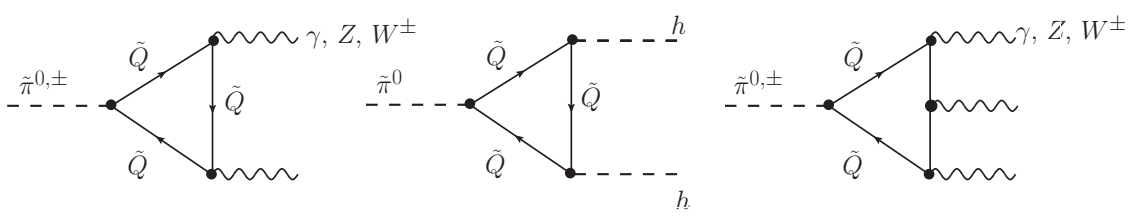

FIG. 20: Light technipion loop-induced (2- and 3-body) decay modes in the leading order through constituent technifermion loops.

In Fig. 18 we show the same observable $\mu_{\gamma \gamma}^{\text {res }}$, but in the minimal CSTC scenario without $\mu_{S, H}$-terms, as a function of $M_{\tilde{\sigma}}$. In opposition to the non-minimal CSTC, in this case there is a very strong dependence on $m_{\tilde{\pi}}$ parameter. Also, in the no mixing limit $s_{\theta} \rightarrow 0$ which corresponds to $M_{\tilde{\sigma}} \rightarrow \sqrt{3} m_{\tilde{\pi}}$, the strength turns to unity $\mu_{\gamma \gamma}^{\text {res }} \rightarrow 1$, as expected, and smearing does not affect this. The current LHC data, in fact, prefer relatively large technipion mass $m_{\tilde{\pi}} \gtrsim 250 \mathrm{GeV}$ and the small $h \tilde{\sigma}$-mixing configuration in the parameter space, and a small vicinity around the "no $h \tilde{\sigma}$-mixing" limit is the only region of parameter space which satisfies the data in the minimal CSTC and the Higgs boson looks as the standard one.

At last, in Fig. 19 we show partial contributions to the Higgs signal strength in the resonance $\mu_{\gamma \gamma}^{\text {res }}$ coming from $W$-loop (dashed lines), top quark loop $\times 10$ (dash-dotted lines), technifermion loop $\times 10$ (dotted lines), technipion loop $\times 1000$ (short-dashed lines), where the rescalings of the curves are made to increase visibility. The shapes of the curves in the minimal and non-minimal CSTC scenarios are very different, but in both cases there is a strong interference pattern.

\section{B. Technipion and technisigma phenomenology}

\section{Technipion decay}

Besides the Higgs boson decay properties studied above, another important phenomenological implication of the CSTC scenario concerns possible technipion and technisigma signatures at the LHC. Since technipions are pseudoscalar particles, at tree level they can be produced only in pairs $\tilde{\pi}^{+} \tilde{\pi}^{-}$or $\tilde{\pi}^{0} \tilde{\pi}^{0}$, which have rather high invariant masses $M_{\tilde{\pi} \tilde{\pi}} \gtrsim 300$ $\mathrm{GeV}$, whereas one-technipion production can be loop induced only (see below). In order to define the phenomenological signatures of technisigma/technipion production at colliders, one has to study primarily the decay modes of produced technipions. In particular, an identification of the produced $\tilde{\pi}$ mesons is important for e.g. studies of the $\tilde{\sigma}$ meson properties 
at the LHC, Yukawa and gauge couplings as well as constituent masses and degeneration of the mass spectrum of the technifermions, etc.
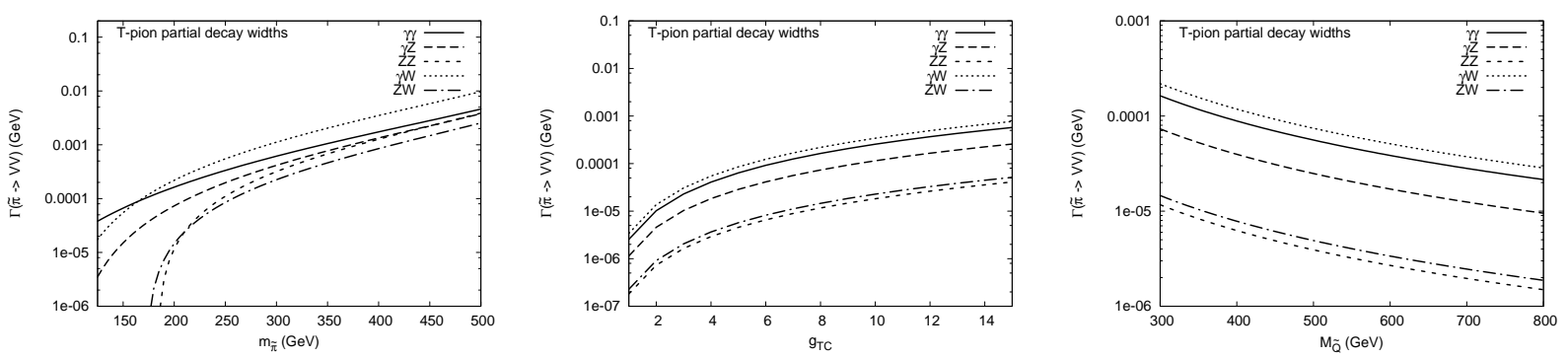

FIG. 21: The technipion decay widths in the loop-induced $\gamma \gamma, \gamma Z, \gamma W, Z Z$ and $Z W$ channels in the non-minimal CSTC (with scalar $\mu_{S, H}$-terms included) as functions of physical parameters of the model. The parameters in each figure are set as follows: (left) $M_{\tilde{Q}}=300 \mathrm{GeV}, c_{\theta}^{2}=0.8$, and $g_{\mathrm{TC}}=8 ;\left(\right.$ middle) $M_{\tilde{Q}}=300 \mathrm{GeV}, m_{\tilde{\pi}}=200 \mathrm{GeV}$, and $c_{\theta}^{2}=0.8 ;$ (right) $m_{\tilde{\pi}}=200 \mathrm{GeV}, c_{\theta}^{2}=0.8$, and $g_{\mathrm{TC}}=8$. These results do not depend on $M_{\tilde{\sigma}}$.

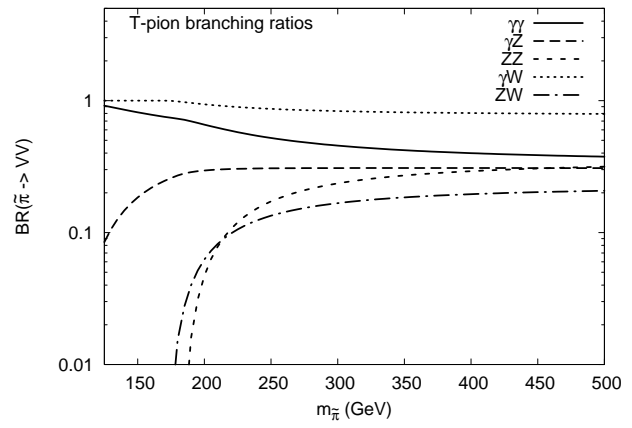

FIG. 22: The neutral and charged technipion branching ratios of the loop-induced $\gamma \gamma, \gamma Z, \gamma W$, $Z Z$ and $Z W$ channels in the non-minimal CSTC (with scalar $\mu_{S, H^{-}}$-terms included) as functions of $m_{\tilde{\pi}}$ for fixed $M_{\tilde{Q}}=300 \mathrm{GeV}, c_{\theta}^{2}=0.8$, and $g_{\mathrm{TC}}=8$.

It is of special interest for collider phenomenology to study $\tilde{\pi}$ decays into vector bosons and, in principle, into a pair Higgs bosons whose diagrams are represented as generic 2- and 3-body technifermion loop-induced processes in Fig. 20. In the case of the mass-degenerated technifermion doublet, it turns out that in the simplest case with $Y_{\tilde{Q}}=0$ the 2-body technipion vector boson decay modes are always forbidden by symmetry encoded in the structure of vertices, whereas allowed for generic $Y_{\tilde{Q}} \neq 0$ cases. The $\tilde{\sigma}$ decays would manifest themselves as multi-lepton final states with a large lepton multiplicity - up to twelve leptons from technipion pair decay in the case of $Y_{\tilde{Q}}=0$ or up to eight leptons for $Y_{\tilde{Q}}=1 / 3$ in the final state from technisigma decay ( six and four leptons coming from each technipion in the above cases, respectively), which would be rather challenging but very interesting to study.

In general, one would deal with many possible four-vector $V V V V$, four-Higgs $h h h h$ or mixed $h h V V$ final states in order to reconstruct the technisigma mass, and this procedure gets even more complicated due a very large $\tilde{\sigma}$ width. If there are no visible deviations of the Higgs boson properties from the SM ones, the technipion/technisigma phenomenology, 
as well as Higgs-scalar self-couplings and studies of various loop-induced processes with the Higgs boson participation, even though very challenging, would be the only source of information about the CSTC sector possibly available at the LHC. The technipion 2-body decay modes into the on-shell gauge bosons, namely, into the $\gamma \gamma, \gamma Z, \gamma W, Z Z$ and $Z W$ final states (above the corresponding thresholds), in the case with $Y_{\tilde{Q}}=1 / 3$ are given by:

$$
\begin{aligned}
& \Gamma\left(\tilde{\pi}^{0} \rightarrow \gamma \gamma\right)=\frac{\alpha^{2} g_{\mathrm{TC}}^{2}}{4 \pi^{3}} \frac{M_{\tilde{Q}}^{2}}{m_{\tilde{\pi}}} \arcsin ^{4}\left(\frac{m_{\tilde{\pi}}}{2 M_{\tilde{Q}}}\right), \quad \frac{m_{\tilde{\pi}}}{2 M_{\tilde{Q}}}<1, \\
& \Gamma\left(\tilde{\pi}^{0} \rightarrow \gamma Z\right)=\frac{\alpha^{2} g_{\mathrm{TC}}^{2}}{2 \pi^{3}} \frac{M_{\tilde{Q}}^{2}}{m_{\tilde{\pi}}} \cot ^{2} 2 \theta_{W}\left(1-\frac{M_{Z}^{2}}{m_{\tilde{\pi}}^{2}}\right)\left[\arcsin ^{2}\left(\frac{m_{\tilde{\pi}}}{2 M_{\tilde{Q}}}\right)-\arcsin ^{2}\left(\frac{M_{Z}}{2 M_{\tilde{Q}}}\right)\right]^{2}, \\
& \Gamma\left(\tilde{\pi}^{ \pm} \rightarrow \gamma W^{ \pm}\right)=\frac{\alpha^{2} g_{\mathrm{TC}}^{2}}{2 \pi^{3} s_{W}^{2}} \frac{M_{\tilde{Q}}^{2}}{m_{\tilde{\pi}}}\left(1-\frac{M_{W}^{2}}{m_{\tilde{\pi}}^{2}}\right)\left[\arcsin ^{2}\left(\frac{m_{\tilde{\pi}}}{2 M_{\tilde{Q}}}\right)-\arcsin ^{2}\left(\frac{M_{W}}{2 M_{\tilde{Q}}}\right)\right]^{2}, \\
& \Gamma\left(\tilde{\pi}^{0} \rightarrow Z Z\right)=\frac{\alpha^{2} g_{\mathrm{TC}}^{2}}{16 \pi^{3}} M_{\tilde{Q}}^{2} m_{\tilde{\pi}}^{3} \bar{\lambda}^{3}\left(M_{Z}^{2}, M_{Z}^{2}, m_{\tilde{\pi}}^{2}\right) C_{0}^{2}\left(M_{Z}^{2}, M_{Z}^{2}, m_{\tilde{\pi}}^{2} ; M_{\tilde{Q}}^{2}\right), \\
& \Gamma\left(\tilde{\pi}^{ \pm} \rightarrow Z W^{ \pm}\right)=\frac{\alpha^{2} g_{\mathrm{TC}}^{2}}{32 \pi^{3} c_{W}^{2}} M_{\tilde{Q}}^{2} m_{\tilde{\pi}}^{3} \bar{\lambda}^{3}\left(M_{Z}^{2}, M_{W}^{2}, m_{\tilde{\pi}}^{2}\right) C_{0}^{2}\left(M_{Z}^{2}, M_{W}^{2}, m_{\tilde{\pi}}^{2} ; M_{\tilde{Q}}^{2}\right),
\end{aligned}
$$

respectively, where the normalized Källen function is defined in Eq. (4.2), and $C_{0}\left(m_{1}^{2}, m_{2}^{2}, m_{3}^{2} ; m^{2}\right) \equiv C_{0}\left(m_{1}^{2}, m_{2}^{2}, m_{3}^{2} ; m^{2}, m^{2}, m^{2}\right)$ is the standard finite three-point function. Note, the $\tilde{\pi}^{0} \rightarrow W W$ decay mode is forbidden by symmetry. The complete set of $\tilde{\pi}$ decay rates (the $\tilde{\pi}^{0} \rightarrow h h$ decay rate which, in principle, exists for heavy technipions vanishes in the "no $h \sigma$-mixing" limit and not included into the analysis) is shown for the non-minimal CSTC scenario in Fig. 21 as functions of the model parameters. The branching
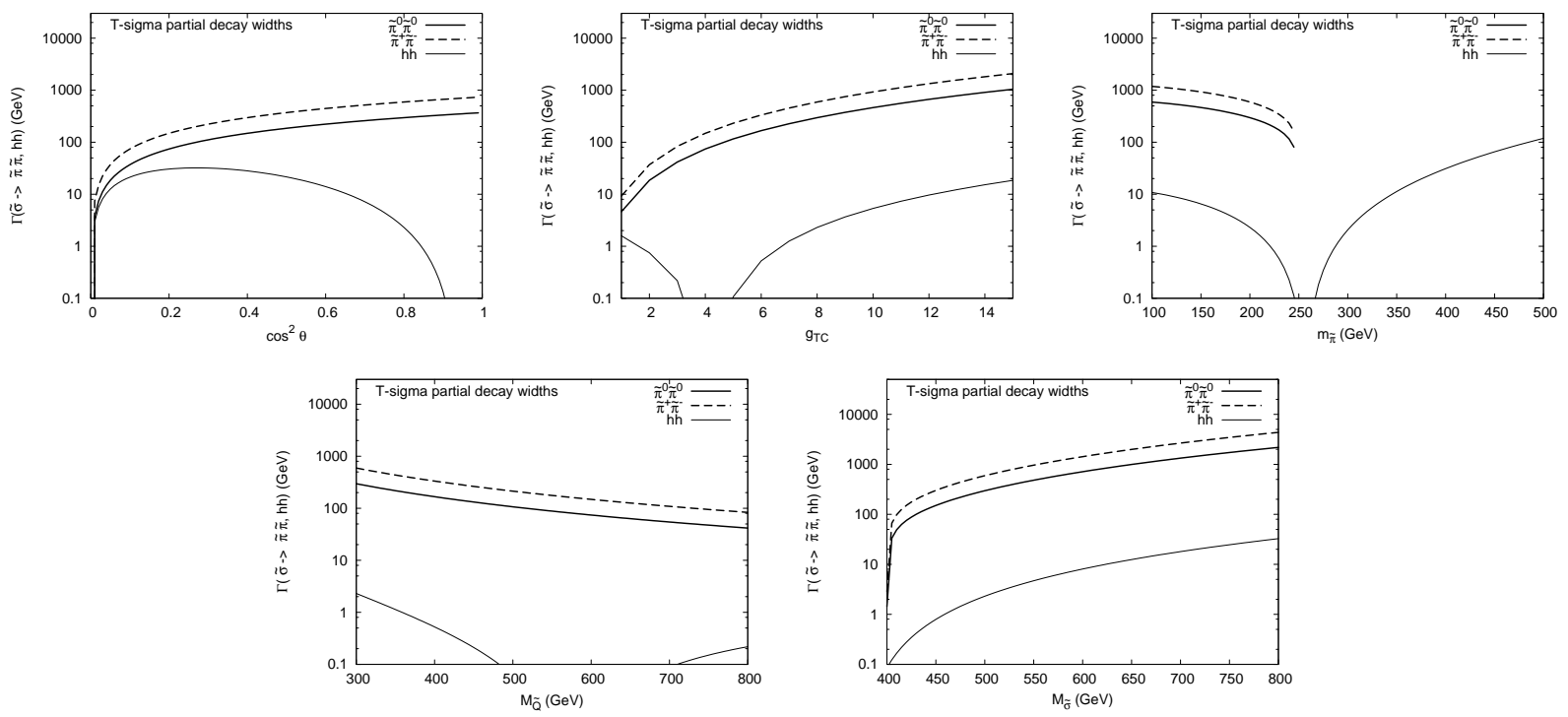

FIG. 23: The technisigma tree-level decay widths in the $\tilde{\pi} \tilde{\pi}$ and $h h$ channels in the non-minimal CSTC (with scalar $\mu_{S, H}$-terms included) as functions of physical parameters of the model. The parameters in each figure are fixed as $m_{\tilde{\pi}}=200 \mathrm{GeV}, M_{\tilde{Q}}=300 \mathrm{GeV}, M_{\tilde{\sigma}}=500 \mathrm{GeV}, c_{\theta}^{2}=0.8$, $g_{\mathrm{TC}}=8$, such that in each figure one drops off a variable from this list corresponding to the one at the respective $x$-axis while keeping others fixed. 
ratios as functions of $m_{\tilde{\pi}}$ at a fixed point in the parameter space as an example are shown in Fig. 22, Interestingly enough, the total technipion decay width is dominated by the $\gamma W^{ \pm}$ channel in the $\tilde{\pi}^{ \pm}$decay, and by the $\gamma \gamma$ channel in the $\tilde{\pi}^{0}$ decay, although other decay modes are not negligible in general.

\section{Technisigma decay}

The tree-level 2-body $\tilde{\sigma}$ decay widths into $\tilde{\pi} \tilde{\pi}, f \bar{f}, Z Z$ and $W W$ are given by the following expressions:

$$
\begin{gathered}
\Gamma(\tilde{\sigma} \rightarrow \tilde{\pi} \tilde{\pi})=\frac{3 g_{\tilde{\sigma} \tilde{\pi}}^{2}}{8 \pi M_{\tilde{\sigma}}} \sqrt{1-\frac{4 m_{\tilde{\pi}}^{2}}{M_{\tilde{\sigma}}^{2}}}, \quad g_{\tilde{\sigma} \tilde{\pi}}=-\lambda_{\mathrm{TC}} u c_{\theta}-\lambda v s_{\theta}, \\
\Gamma(\tilde{\sigma} \rightarrow \bar{f} f)=\frac{g^{2} s_{\theta}^{2}}{32 \pi} M_{\tilde{\sigma}} \frac{M_{f}^{2}}{M_{W}^{2}}\left(1-\frac{4 M_{f}^{2}}{M_{\tilde{\sigma}}^{2}}\right)^{3 / 2}, \\
\Gamma(\tilde{\sigma} \rightarrow Z Z)=\frac{g^{2} s_{\theta}^{2}}{16 \pi} \frac{M_{Z}^{2}}{M_{\tilde{\sigma}} c_{W}^{2}}\left(1-\frac{4 M_{Z}^{2}}{M_{\tilde{\sigma}}^{2}}\right)^{1 / 2} \cdot\left[1+\frac{\left(M_{\tilde{\sigma}}^{2}-2 M_{Z}^{2}\right)^{2}}{8 M_{Z}^{4}}\right], \\
\Gamma(\tilde{\sigma} \rightarrow W W)=\frac{g^{2} s_{\theta}^{2}}{8 \pi} \frac{M_{W}^{2}}{M_{\tilde{\sigma}}}\left(1-\frac{4 M_{W}^{2}}{M_{\tilde{\sigma}}^{2}}\right)^{1 / 2} \cdot\left[1+\frac{\left(M_{\tilde{\sigma}}^{2}-2 M_{W}^{2}\right)^{2}}{8 M_{W}^{4}}\right],
\end{gathered}
$$

respectively, while the loop-induced $\tilde{\sigma}$ decay widths in the $\gamma \gamma$ and $\gamma Z$ channels can be
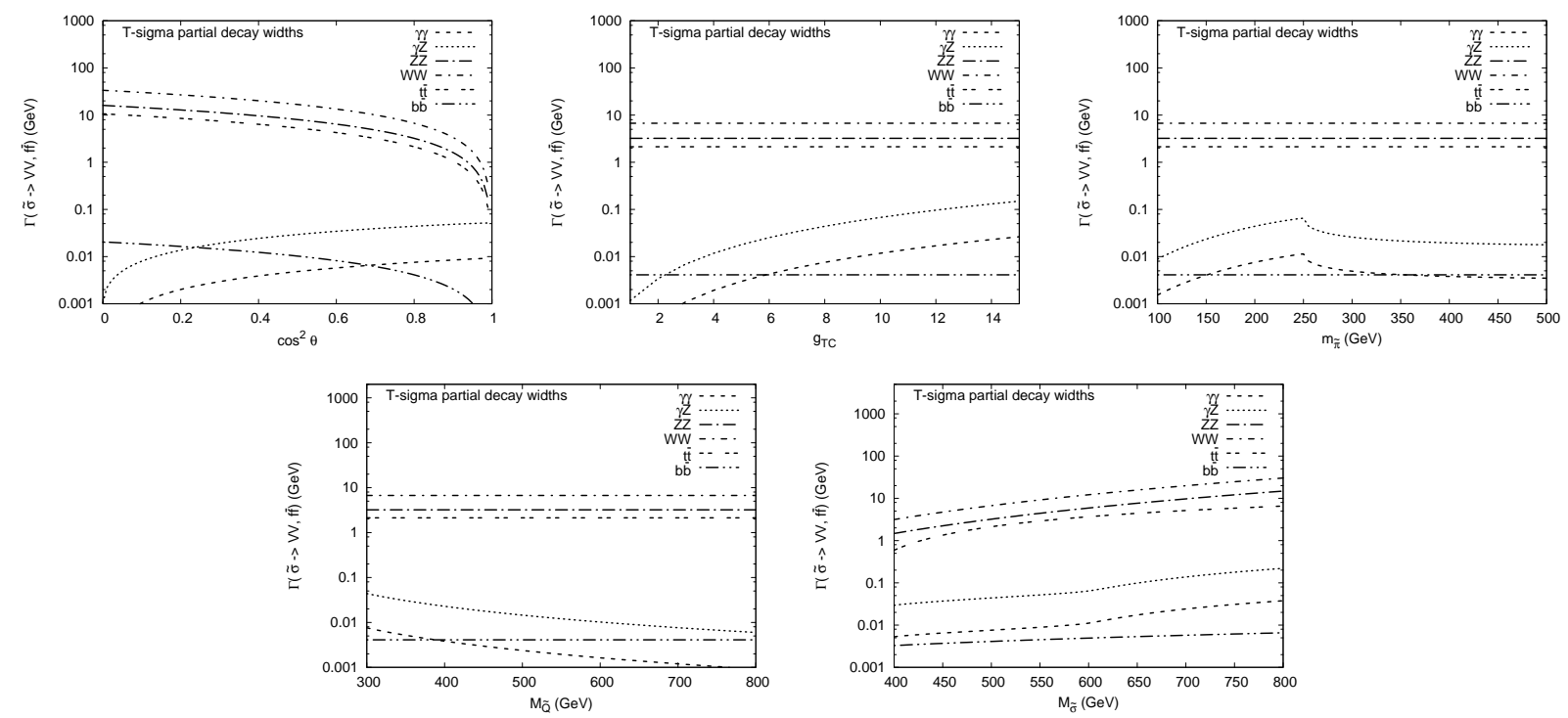

FIG. 24: The technisigma tree-level decay widths in the fermion $(t \bar{t}, b \bar{b})$ and gauge boson $(\gamma \gamma, \gamma Z$, $Z Z$ and $W W$ ) channels in the non-minimal CSTC (with scalar $\mu_{S, H}$-terms included) as functions of physical parameters of the model. The set-up of parameters is the same as in Fig. 23 .

obtained from that of the Higgs boson by a replacement $c_{\theta} \rightarrow s_{\theta}, M_{h} \rightarrow M_{\tilde{\sigma}}$, and thus are not shown here explicitly. The (pseudo)scalar ( $h h$ and $\tilde{\pi} \tilde{\pi}$ ) decay modes are shown for the non-minimal CSTC scenario in Fig. 23 as functions of the model parameters, while 
fermion $(t \bar{t}, b \bar{b})$ and gauge boson $(\gamma \gamma, \gamma Z, Z Z$ and $W W)$ decay channels are given in Fig. 24. One notices that the technipion modes of the $\tilde{\sigma}$ decay strongly dominate the total $\tilde{\sigma}$ decay width, and can be as large as a few hundreds $\mathrm{GeV}$ being comparable to $M_{\tilde{\sigma}}$. Certainly, $\tilde{\sigma}$ is a highly unstable and unusually broad state, for which one cannot use the narrow width approximation, and it is an open question how to identify it experimentally.

\section{One-technipion production}

As has been mentioned above, one technipion can be produced only at the loop level. Let us look into this possibility in more detail since this channel is especially important for understanding the discovery potential of Technicolor at the LHC, even in the absence of any deviations the Higgs boson signal strengths from the SM predictions. Corresponding typical partonic $2 \rightarrow 3$ hard subprocess of Higgs boson and $\tilde{\pi}$ production in high energy hadron-hadron collisions via intermediate vector boson fusion (VBF) mechanism is shown in Fig. 25, The Higgs boson VBF production channel (left panel) shown for comparison with
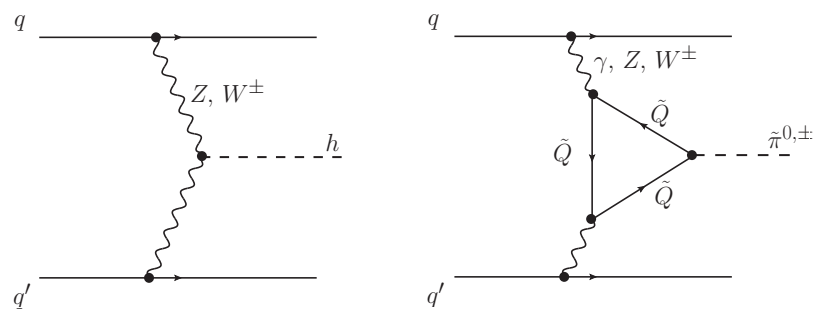

FIG. 25: Typical production channels of the Higgs boson at tree level (left) and technipion via a triangle technifermion loop (right) via gauge boson fusion in the quark-(anti)quark scattering.

the technipion channel (right panel) is one of the key production modes recently studied at the LHC which allowed for clear discrimination of the Higgs signal and large backgrounds [1, 2]. The Higgs boson has also other production modes e.g. via gluon-gluon fusion mechanism and the Higgsstrahlung off gauge bosons and heavy flavor. In opposite to the Higgs boson, one technipion can only be produced via heavy technifermion triangle loop in the VBF mechanism. In numerical estimations, it is explicitly assumed that the incoming quark $q$ and (anti)quark $q^{\prime}$ loose only a small fraction of their initial energy taken away by intermediate vector bosons. In this kinematics, the final-state quarks are seen as forward-backward hard jets, and by measuring their momenta one accurately reconstructs the invariant mass of the produced state. An overall one-technipion production rate is expected to be strongly suppressed compared to the Higgs boson production rate, which along with extremely narrow technipion resonance makes it rather hard to measure experimentally but not impossible.

In Fig. 26 we show the one-technipion production cross sections via the VBF mechanism at the parton level for different incoming and outgoing quark $q$ and (anti)quark $q^{\prime}$ states. Both parton-level and hadron-level cross sections at the LHC with $\sqrt{s}=14 \mathrm{TeV}$ in the relevant kinematics and mass ranges along with corresponding Higgs boson cross sections in respective channels are presented (here and below, CTEQ5LO quark PDFs [52] were used in calculations). Only up and down quarks with at least one valence quark as well as contributions with maximal Cabibbo-Kobayashi-Maskawa mixing terms are included here. We have not applied any detector cuts or hadronisation corrections here, which would be the next crucial step in phenomenological studies of the CSTC model. All the numerical 
estimates here are done for the first time in order to understand the potential of the suggested model. Even for a rather large technifermion-technipion coupling $g_{\mathrm{TC}}=8$ we observe that the hadronic cross sections of the technipion production (middle panel) by about two orders of magnitude smaller than those for the Higgs boson (right panel) in the same masse range. This suppression will be even stronger for smaller $g_{\mathrm{TC}}$ coupling and does not depend on other CSTC model parameters. The respective production mechanism is thus one of the "golden" channels for technipion and, in general, new strongly-coupled sector searches at the LHC in measurements with high statistics.
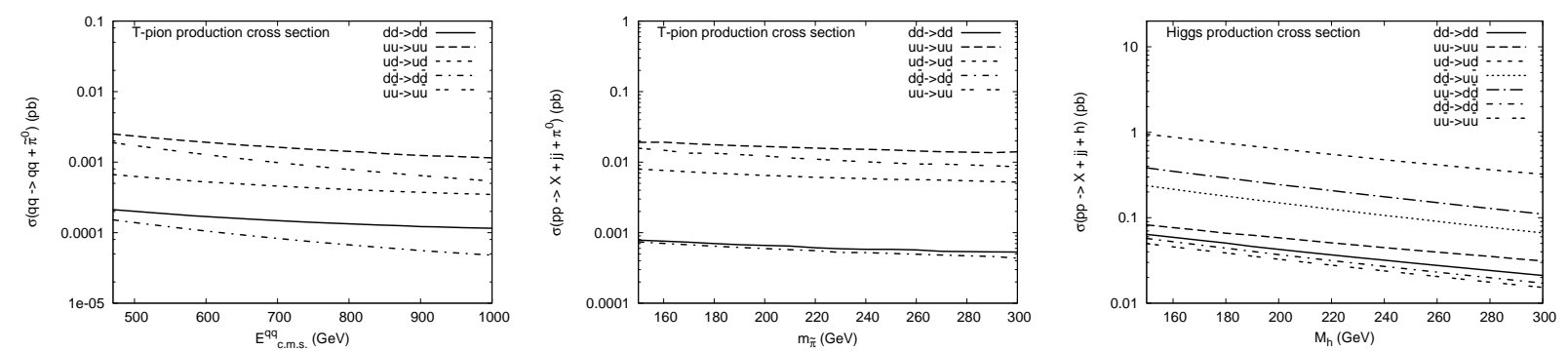

FIG. 26: The one-technipion (T-pion) production cross sections via the VBF mechanism at the parton level for different incoming and outgoing quark $q$ and (anti)quark $q^{\prime}$ states as functions of $q q^{\prime}$ invariant mass, or c.m.s. energy $E_{c . m . s .}^{q q}=\sqrt{\hat{s}}$ (left), corresponding total hadron level cross sections of one technipion production for given incoming $q q^{\prime}$ states in picobarns (before cuts) at the maximal LHC energy $\sqrt{s}=14 \mathrm{TeV}$ as a function of the technipion mass $m_{\tilde{\pi}}$ (middle), and corresponding VBF hadronic cross sections of the Higgs boson as functions of its mass $M_{h}$ shown for comparison. Here, $g_{\mathrm{TC}}=8$ and $M_{\tilde{Q}}=300 \mathrm{GeV}$ are fixed, and the results do not depend on other CSTC parameters. In calculations of the hadronic cross sections in this paper we have used quark CTEQ5LO PDFs [52].

The discovery potential depends also on the subsequent decay modes and branching ratios of technipions. As was demonstrated above, the decay modes of the neutral technipion are similar to the vector-boson decay modes of the Higgs boson including $\gamma \gamma, Z Z$ and $\gamma Z$ channels, however, $\tilde{\pi}^{0} \rightarrow W^{+} W^{-}$mode is forbidden by symmetry. In the range of relatively small $m_{\tilde{\pi}} \lesssim 200 \mathrm{GeV}$ the strategy for searches of technipions will be similar to that in the Higgs boson searches. Moreover, for light technipions it turns out that the $\gamma \gamma$ signals from the Higgs boson and technipion can be comparable with each other due to a very small $\gamma \gamma$ branching ratio of the Higgs boson $\operatorname{BR}(h \rightarrow \gamma \gamma) \simeq 10^{-3}$, while corresponding technipion branching is relatively large $\operatorname{BR}(\tilde{\pi} \rightarrow \gamma \gamma) \simeq 0.5-1.0$ (see Fig. 22). The issue with detection of such light technipions in the $\gamma \gamma$ or $\gamma Z$ channels can arise, however, due to a very narrow technipion resonance since in the mass range $\sim 150 \mathrm{GeV}$ the total technipion decay width amounts to $\lesssim 0.1 \mathrm{MeV}$ (see Fig. 21). Such an extremely narrow resonance, in principle, can be missed in the Higgs-type searches at the LHC, and an additional investigation of this possibility is necessary. Also, a possibility of a relative proximity or even an overlap of the Higgs resonance and extremely narrow technipion resonance is not completely excluded, and remains to be an interesting opportunity. Further, a more elaborate analysis and the search for light technipions in the existing LHC data is required.

At last, heavier technipions $m_{\tilde{\pi}} \gtrsim 200 \mathrm{GeV}$ can be searched for in the $\gamma \gamma, \gamma Z$ and $Z Z$ decay channels which have comparable branchings. The dominant modes for the heavy Higgs boson searches are typically $W W$ and $Z Z$ ones with large branchings, whereas $\gamma \gamma$, 
$\gamma Z$ branchings of the Higgs decay are practically zeroth. The only common channel for technipion and Higgs boson in the high mass range is the $Z Z$ one. However, having comparable branchings, the technipion production rate is strongly suppressed compared to that of the Higgs boson (see above). So, the current LHC statistics may not be enough for establishing significant constraints onto the CSTC model parameter space for the higher technipion masses, and further studies are certainly needed.

\section{Technipion pair production}

Typical leading-order (tree-level) processes of the $\tilde{\pi}$-pair production in $f \bar{f}$ and vector boson fusion at the LHC are shown in Fig. 27. Besides rather high $\tilde{\pi} \tilde{\pi}$ pair invariant mass $M_{\tilde{\pi} \tilde{\pi}} \gtrsim 300 \mathrm{GeV}$, an additional suppression in $V V$ and $f \bar{f}$ production channels appear due to rather weak couplings $g$ and $g^{2}$ in $\tilde{\pi} \tilde{\pi} V$ and $\tilde{\pi} \tilde{\pi} V V$ vertices respectively (cf. Eq. (2.26)), as well as due to a large off-resonant suppression in $s$-channel subprocesses with intermediate Higgs and gauge bosons, which are much lightest than $M_{\tilde{\pi} \tilde{\pi}}$.

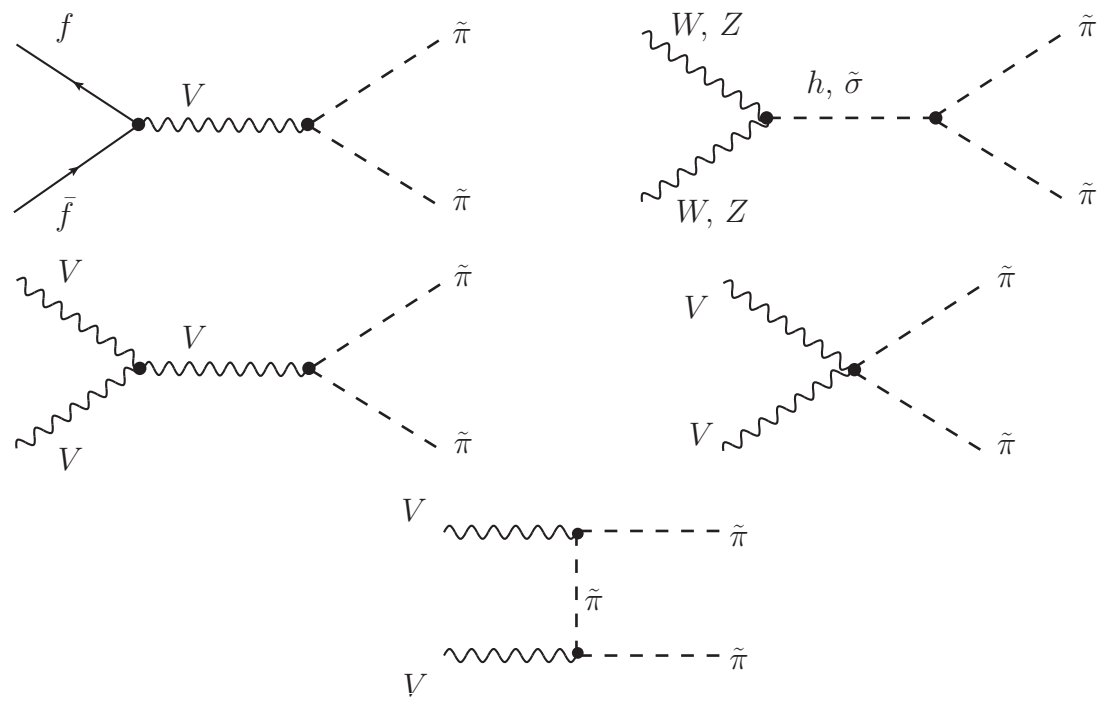

FIG. 27: Typical technipion production channels in the leading order, relevant for collider phenomenology. Here, $V=Z, W, \gamma$ in appropriate places. The $g g h$ and $g g \tilde{\sigma}$ couplings are heavy quark loop-induced ones in the leading order.

Thus, one may naively assume that the largest contribution to the $\tilde{\pi}^{+} \tilde{\pi}^{-}$and $\tilde{\pi}^{0} \tilde{\pi}^{0}$ production rates comes essentially from the intermediate technisigma resonance with the $\tilde{\pi} \tilde{\pi} \tilde{\sigma}$ coupling

$$
g_{\tilde{\pi} \tilde{\pi} \tilde{\sigma}}=-g_{\mathrm{TC}} c_{\theta} \frac{M_{\tilde{\sigma}}^{2}-m_{\tilde{\pi}}^{2}}{2 M_{\tilde{Q}}}
$$

which is not suppressed in the small mixing limit (for not very heavy technifermions). However, in the latter case one encounters more sources of suppression. Firstly, the production rate of the $\tilde{\sigma}$ itself in the SM-like channels most likely to be suppressed by a small mixing angle, i.e. by the $s_{\theta}^{2} \ll 1$ factor in the cross section, compared to the Higgs boson production rate with $c_{\theta}^{2} \sim 1$ (see the previous Section). Secondly, the $\tilde{\sigma}$ total decay width dominated 
by the technipion channel (in analogy to hadron physics) is typically large, of the order of a few hundreds of $\mathrm{GeV}$, which means that there will be no any resonant enhancement in the $\tilde{\pi} \tilde{\pi}$ production rate associated with the technisigma channel. Thus, overall rates of the tree-level $\tilde{\sigma}$ and $\tilde{\pi} \tilde{\pi}$ production are expected to be rather small, similarly to the loop-induced one technipion rates calculated above. Moreover, in the small mixing or "no $h \tilde{\sigma}$-mixing" scenario the only possible $\tilde{\sigma}$ production channel is through the gauge boson fusion through the technifermion and technipion triangles since the $\tilde{Q} \tilde{Q} \tilde{\sigma}$ coupling (2.25) is finite

$$
g_{\tilde{Q} \tilde{Q} \tilde{\sigma}}=-g_{\mathrm{TC}} c_{\theta},
$$

and can be rather large due to the "fat" TC coupling $g_{\mathrm{TC}}>1$. Besides the dominant technisigma decay mode, the $\tilde{\pi} \tilde{\pi}$ pair may also be produced at one loop level via $\tilde{Q}$ box diagrams. These details of the lightest technihadron dynamics would make the search for new technipion/technisigma states to be rather challenging at the LHC, but not impossible.
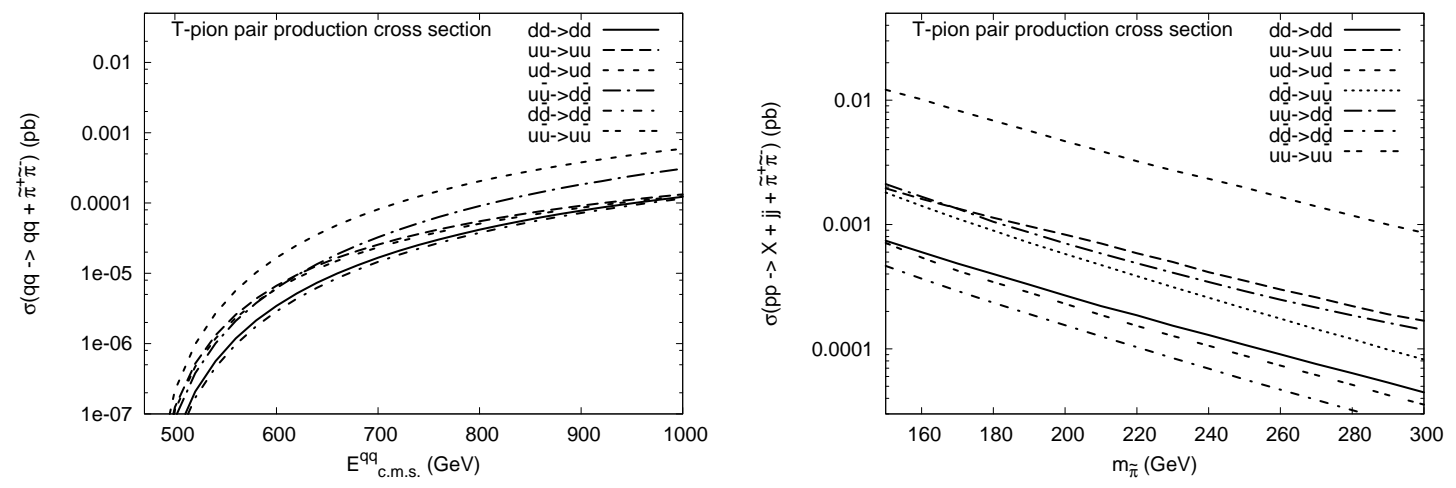

FIG. 28: The one-technipion (T-pion) pair production cross sections via the VBF mechanism at the parton level for different incoming and outgoing quark $q$ and (anti)quark $q^{\prime}$ states as functions of $q q^{\prime}$ invariant mass, or c.m.s. energy $E_{c . m . s .}^{q q}=\sqrt{\hat{s}}$ (left), corresponding total hadron level cross sections of the technipion pair production for given incoming $q q^{\prime}$ states in picobarns (before cuts) at the maximal LHC energy $\sqrt{s}=14 \mathrm{TeV}$ as a function of the technipion mass $m_{\tilde{\pi}}$ (right). Here, $g_{\mathrm{TC}}=8, c_{\theta}^{2}=0.8, M_{\tilde{\sigma}}=600 \mathrm{GeV}$ and $M_{\tilde{Q}}=300 \mathrm{GeV}$ are fixed.

For illustration, in Fig. 28 we present the $\tilde{\pi}^{+} \tilde{\pi}^{-}$pair production cross sections at the parton level in the VBF mechanism as functions of the $q q^{\prime}$ center-of-mass energy for different initial and final quarks (left) and the corresponding hadron-level cross sections at the LHC $(\sqrt{s}=14 \mathrm{TeV})$ as functions the technipion mass (right). The quark-antiquark fusion mechanism going via $h$ or $\tilde{\sigma}$ resonance is assumed to be negligible in the forward/backward jets kinematics considered here and was not included in this calculation. In opposite to the one-technipion production cross sections shown in Fig. 26, the parton-level $\tilde{\pi}^{+} \tilde{\pi}^{-}$pair production cross sections increase at higher $q q^{\prime}$ c.m.s. energies (or larger quark fractions $x$ ) and can reach the same magnitudes as the one-technipion cross sections at $E_{c . m . s .}^{q q} \gtrsim 700$ $\mathrm{GeV}$. The hadronic $\tilde{\pi}^{+} \tilde{\pi}^{-}$cross sections drop faster than corresponding one-technipion cross sections and have similar order-of-magnitude values for the light $\tilde{\pi}$ mass range. This means that both one- and two-technipion processes should be studied on the same footing. The latter, however, would be more difficult to identify experimentally due to a larger multiplicity of leptons and tiny widths of the technipions. 


\section{SUMMARY}

To summarize, in this work we have constructed and investigated in major details the chiral-symmetric (vector-like) Technicolor scenario, according to which a new sector of technifermions in confinement interacts with the SM gauge bosons by means of vector-like gauge couplings. Our analysis is based upon the gauged linear $\sigma$-model with initially global chiralgauge $S U(2)_{\mathrm{L}} \otimes S U(2)_{\mathrm{R}}$ group broken down to the local LR-symmetric SM weak isospin symmetry $S U(2)_{L+R \equiv W}$ group in the technifermion sector.

The Higgs boson in this scenario is considered as a separate (fundamental or composite) scalar state and introduced in the same way as in the one-doublet SM. Nevertheless, we have shown that the electro-weak symmetry breaking at the scale $M_{\mathrm{EW}} \sim 100 \mathrm{GeV}$ can be initiated dynamically by the presence of the confined vector-like technifermion sector, namely, it is triggered by the technifermion condensate at the techniconfinement scale, $\Lambda_{\mathrm{TC}} \gtrsim$ $M_{\mathrm{EW}}$, together with the chiral symmetry breaking. This thus leads to the effective SM Higgs mechanism of dynamical electro-weak symmetry breaking.

Remarkably, this model is well consistent with both EW precision constraints and, simultaneously, with the recent SM-like Higgs boson observations at the LHC in the small Higgs-technisigma mixing limit. At the same time, the model predicts the existence of extra new lightest technihadron states, namely, physical technipions $\tilde{\pi}$ and technisigma $\tilde{\sigma}$, at the LHC energy scales, giving rise to rich Technicolor phenomenology at the LHC. Detection prospects for these new states have also been discussed, and the most phenomenologically important decay modes of $\tilde{\pi}$ and $\tilde{\sigma}$, as well as technipion production cross sections, were quantified over physically reasonable regions of parameter space.

In the absence of noticeable deviations from the SM predictions in the Higgs signal strengths, the suggested scenario is capable of explaining of what triggers the SM Higgs mechanism, the nature of the Higgs vev in the nearly-conformal limit of the new stronglycoupled dynamics. The proposed vector-like Technicolor scenario, in its simplest form considered here, does not attempt to resolve the naturalness problem of the SM, i.e. does not provide a mechanism protecting the Higgs boson mass itself from becoming arbitrary large. Nevertheless, this minimal realization of the TC ideas preserving the effective Higgs mechanism of the SM opens up new prospects for more elaborated scenarios with extended chiral-gauge groups possibly predicting the light composite Higgs boson(s) with well-defined vector-like ultraviolet completion, which is the subject of our further analysis. At last, as a specific prediction of this class of models, the lightest neutral heavy weakly-interacting technibaryon state gives rise to a suitable Dark Matter candidate making it to be especially attractive opportunity for astrophysical New Physics searches, and a corresponding analysis is planned for future studies.

\section{Acknowledgments}

Stimulating discussions and helpful correspondence with Johan Bijnens, Gabriele Ferretti, Stefano Frixione, Christophe Grojean, Giuliano Panico, Sabir Ramazanov, Johan Rathsman, Slava Rychkov, Francesco Sannino, Torbjörn Sjöstrand and Peter Skands are gratefully acknowledged. This work was supported in part by the Crafoord Foundation (Grant No. 20120520). R.P. is thankful to the CERN Theory Group for support and inspiring discussions

during his visit at CERN. V.K. is especially grateful to the Lund THEP Group for support 
and hospitality during his visit at Lund University at the final stage of this work.

[1] G. Aad et al. [ATLAS Collaboration], Phys. Lett. B 716, 1 (2012) arXiv:1207.7214 [hep-ex]]; Science 338, 1576 (2012).

[2] S. Chatrchyan et al. [CMS Collaboration], Phys. Lett. B 716, 30 (2012) arXiv:1207.7235 [hep-ex]];

Science 338, 1569 (2012).

[3] T. Aaltonen et al. [CDF and D0 Collaborations], Phys. Rev. Lett. 109, 071804 (2012) arXiv:1207.6436 [hep-ex]].

[4] The ATLAS collaboration, ATLAS-CONF-2013-014;

The ATLAS collaboration, ATLAS-CONF-2013-034;

The CMS collaboration, CMS PAS-HIG-12-045;

The CMS collaboration, CMS PAS-HIG-13-001.

[5] T. Aaltonen et al. [CDF and D0 Collaborations], arXiv:1303.6346 [hep-ex].

[6] A. Falkowski, F. Riva, A. Urbano, arXiv:1303.1812 [hep-ph].

[7] J. Ellis and T. You, arXiv:1303.3879 [hep-ph].

[8] A. Djouadi and G. Moreau, arXiv:1303.6591 [hep-ph].

[9] P. P. Giardino, K. Kannike, I. Masina, M. Raidal, A. Strumia, arXiv:1303.3570 [hep-ph].

[10] T. Alanne, S. Di Chiara and K. Tuominen, arXiv:1303.3615 [hep-ph].

[11] K. Cheung, J. S. Lee, P. -Y. Tseng, arXiv:1302.3794 [hep-ph].

[12] S. Dittmaier et al. [LHC Higgs Cross Section Working Group Collaboration], arXiv:1101.0593 [hep-ph]; arXiv:1201.3084 [hep-ph].

[13] S. Weinberg, Phys. Rev. D 13, 974 (1976);

L. Susskind, Phys. Rev. D 20, 2619 (1979).

[14] S. Dimopoulos and L. Susskind, Nucl. Phys. B 155, 237 (1979);

E. Eichten and K. D. Lane, Phys. Lett. B 90, 125 (1980).

[15] M. E. Peskin and T. Takeuchi, Phys. Rev. Lett. 65, 964 (1990);

M. E. Peskin, T. Takeuchi, Phys. Rev. D 46, 381 (1992).

[16] C. T. Hill and E. H. Simmons, Phys. Rept. 381, 235 (2003) [Erratum-ibid. 390, 553 (2004)] hep-ph/0203079.

[17] F. Sannino, Acta Phys. Polon. B 40, 3533 (2009) [arXiv:0911.0931 [hep-ph]].

[18] T. W. Appelquist, D. Karabali, L. C. R. Wijewardhana, Phys. Rev. Lett. 57, 957 (1986).

[19] F. Sannino and K. Tuominen, Phys. Rev. D 71, 051901 (2005) hep-ph/0405209.

[20] R. Foadi, M. T. Frandsen, T. A. Ryttov, F. Sannino, Phys. Rev. D 76, 055005 (2007) arXiv:0706.1696 [hep-ph]].

[21] E. H. Simmons, Nucl. Phys. B 312, 253 (1989).

[22] S. Samuel, Nucl. Phys. B 347, 625 (1990).

[23] A. Kagan and S. Samuel, Phys. Lett. B 270, 37 (1991).

[24] C. D. Carone, J. Erlich, J. A. Tan, Phys. Rev. D 75, 075005 (2007) hep-ph/0612242.

[25] C. D. Carone, Phys. Rev. D 86, 055011 (2012) arXiv:1206.4324 [hep-ph]].

[26] K. Agashe, R. Contino, A. Pomarol, Nucl. Phys. B 719, 165 (2005) hep-ph/0412089.

[27] J. Hirn, V. Sanz, Phys. Rev. Lett. 97, 121803 (2006) hep-ph/0606086].

[28] D. K. Hong, H. -U. Yee, Phys. Rev. D 74, 015011 (2006) hep-ph/0602177.

[29] R. S. Chivukula, hep-ph/0011264. 
[30] M. Green, J. Schwarz, Phys. Lett. B149, 117 (1984).

[31] H. Harari, M. Leurer, Nucl. Phys. B 233, 221 (1984).

[32] Y. Nambu and G. Jona-Lasinio, Phys. Rev. 122, 345 (1961);

D. Ebert and M. K. Volkov, Z. Phys. C 16, 205 (1983);

M. K. Volkov, Ann. Phys. 157, 282 (1984).

[33] U. Vogl, W. Weise, Prog. Part. Nucl. Phys. 27, 195 (1991).

[34] W.A. Bardeen, C.N. Leung, and S.T. Love, Phys. Rev. Lett. 56, 1230 (1986);

C.N. Leung, S.T. Love, and W.A. Bardeen, Nucl. Phys. B 273, 649 (1986);

M. Harada, Y. Kikukawa, T. Kugo, H. Nakano, Prog. Theor. Phys. 92, 1161 (1994) hep-ph/9407398.

[35] V.A. Miransky, Dynamical Symmetry Breaking in Quantum Field Theories, World-Scientific, Singapore, 1993.

[36] B.W. Lee and H.T. Nieh, Phys. Rev. 166, 1507 (1968).

[37] S. Gasiorowicz and D. Geffen, Rev. Mod. Phys. 41, 531 (1969);

P. Ko and S. Rudaz, Phys. Rev. D 50, 6877 (1994);

M. Urban, M. Buballa, and J. Wambach, Nucl. Phys. A697, 338 (2002).

[38] B.D. Serot and J.D. Walecka, Acta Phys. Pol. B 21, 655 (1992).

[39] T. Eguchi, Phys. Rev. D 14, 2755 (1976);

K. Kikkawa, Prog. Theor. Phys. 56, 947 (1976);

M. K. Volkov, Sov. J. Part. Nucl. 17, 186 (1986) [Fiz. Elem. Chast. Atom. Yadra 17, 433 (1986)].

[40] B. D. Serot, Phys. Lett. B 86, 146 (1979) [Erratum-ibid. B 87, 403 (1979)].

[41] U.-G. Meibner, Phys. Rep. 161, 214 (1988);

B. D. Serot, J. D. Walecka, Int. J. Mod. Phys. E 6, 515 (1997) nucl-th/9701058].

[42] D. V. Shirkov and I. L. Solovtsov, Phys. Rev. Lett. 79, 1209 (1997) hep-ph/9704333.

[43] M. A. Shifman, A. I. Vainshtein and V. I. Zakharov, Nucl. Phys. B 147, 385 (1979).

[44] I. Maksymyk, C. P. Burgess, D. London, Phys. Rev. D 50, 529 (1994) hep-ph/9306267.

[45] C. P. Burgess, S. Godfrey, H. Konig, D. London, I. Maksymyk, Phys. Lett. B 326, 276 (1994) hep-ph/9307337.

[46] J. Beringer et al. (Particle Data Group), Phys. Rev. D86, 010001 (2012).

[47] R. Barbieri, A. Pomarol, R. Rattazzi, A. Strumia, Nucl. Phys. B 703, 127 (2004) hep-ph/0405040.

[48] B. A. Kniehl, Phys. Rept. 240, 211 (1994).

[49] G. Passarino and M. J. G. Veltman, Nucl. Phys. B160, 151 (1979).

[50] H. S. Fukano, F. Sannino, Int. J. Mod. Phys. A 25, 3911 (2010) [arXiv:0908.2424 [hep-ph]].

[51] V. I. Kuksa, Phys. Lett. B 633, 545 (2006) [Erratum-ibid. B 664, 315 (2008)] hep-ph/0508164; Int. J. Mod. Phys. A 23, 4509 (2008) [Erratum-ibid. 24, 4221 (2009)] arXiv:0706.0311 [hep-ph]].

[52] H. L. Lai et al. [CTEQ Collaboration], Eur. Phys. J. C 12, 375 (2000) hep-ph/9903282. 